Beiträge aus der Nordwestdeutschen Forstlichen Versuchsanstalt

Kohlenstoffstudie Forst und Holz

Niedersachsen

René Wördehoff, Hermann Spellmann, Jan Evers, Jürgen Nagel
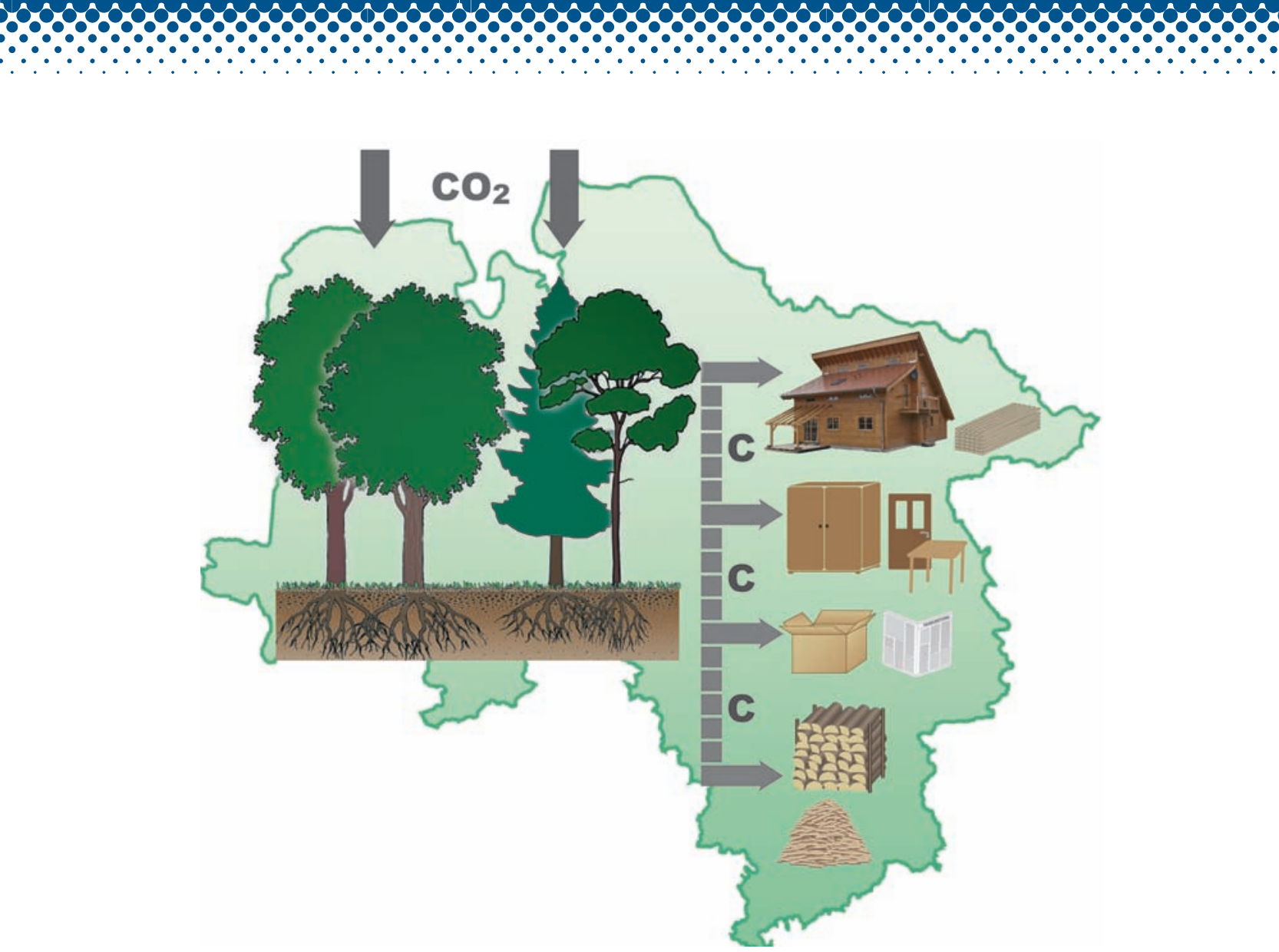

Gnt Universitätsdrucke Göttingen

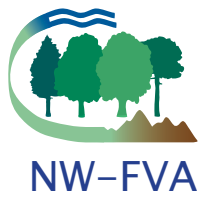



René Wördehoff, Hermann Spellmann, Jan Evers, Jürgen Nagel Kohlenstoffstudie Forst und Holz Niedersachsen 
erschienen als Band 6 der Reihe „Beiträge aus der Nordwestdeutschen Forstlichen Versuchsanstalt“ in den Universitätsdrucken im Universitätsverlag Göttingen 2011 
René Wördehoff, Hermann Spellmann, Jan Evers, Jürgen Nagel

\section{Kohlenstoffstudie Forst und Holz Niedersachsen}

Beiträge aus der

Nordwestdeutschen

Forstlichen Versuchsanstalt

Band 6

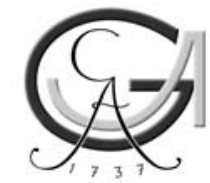

Universitätsverlag Göttingen 2011 


\section{Bibliographische Information der Deutschen Nationalbibliothek}

Die Deutsche Nationalbibliothek verzeichnet diese Publikation in der Deutschen Nationalbibliographie; detaillierte bibliographische Daten sind im Internet über $<$ http://dnb.ddb.de> abrufbar.

Global Forest Decimal Classification: 111.83, 161.32, 2, 564, 907.33

\section{Herausgeber}

Nordwestdeutsche Forstliche Versuchsanstalt (NW-FVA)

Grätzelstr. 2, D-37079 Göttingen

Tel.: +49 (0)551-69401-0, Fax: +49 (0)551-69401-160

E-Mail: zentrale@nw-fva.de

www.nw-fva.de

Schriftleitung der Reihe: Prof. Dr. Hermann Spellmann

Redaktion: Inge Kehr, Ulrike Gaertner

Die Kohlenstoffstudie Forst und Holz Niedersachsen wurde vom

Niedersächsischen Ministerium für Ernährung, Landwirtschaft,

Verbraucherschutz und Landesentwicklung finanziert.

Titelgrafik und Abbildung 26: Etta Paar (NW-FVA)

Dieses Buch ist auch als freie Onlineversion über die Homepage der NW-FVA, des Verlages sowie über den OPAC der Niedersächsischen Staats- und Universitätsbibliothek (http://www.sub.uni-goettingen.de) erreichbar und darf gelesen, heruntergeladen sowie als Privatkopie ausgedruckt werden. Es gelten die Lizenzbestimmungen der Onlineversion. Es ist nicht gestattet, Kopien oder gedruckte Fassungen der freien Onlineversion zu veräußern.

(C) 2011 Universitätsverlag Göttingen

http://univerlag.uni-goettingen.de

ISBN: 978-3-86395-014-9

ISSN: $1865-6994$ 


\section{Vorwort}

Der Wald in Niedersachsen ist mit einem Anteil von $24 \%$ an der Landesfläche bzw. 1,1 Millionen Hektar ein prägendes Landschaftselement. Neben den Schutz- und Erholungsfunktionen liefert er den vielseitig verwendbaren Bau- und Werkstoff Holz, bei dessen Produktion das klimaschädliche Treibhausgas $\mathrm{CO}_{2}$ als Kohlenstoff gebunden und als Sauerstoff freigesetzt wird.

Auf der Grundlage der Clusteranalyse Forst und Holz Niedersachsen aus dem Jahr 2007 ist das Ziel der Kohlenstoffstudie, die Bedeutung der Gesamtbranche für eine optimale Bindung von $\mathrm{CO}_{2}$ am Beispiel Niedersachsens aufzuzeigen. Bisher war die Fragestellung in der bundesweiten Diskussion verstärkt auf die Betrachtung des Waldes allein ausgerichtet. Aber

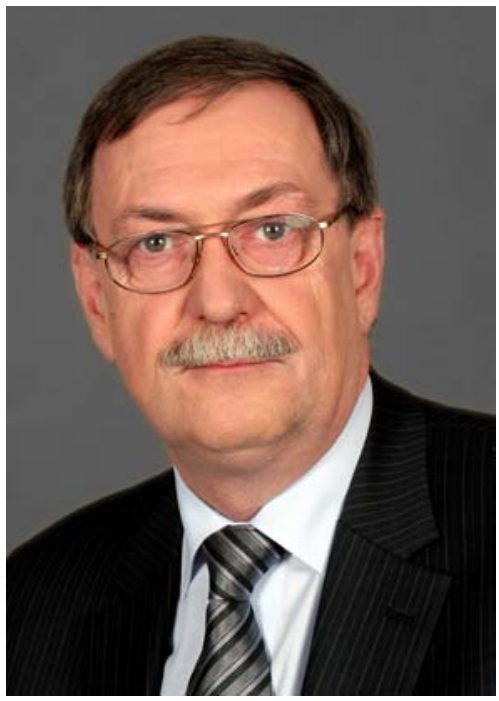
erst die Einbeziehung der langfristigen Verwendung und damit der $\mathrm{CO}_{2}$-Bindung in Holzprodukten macht die überragende Rolle des Clusters Forst- und Holzwirtschaft für den Klimaschutz deutlich.

Die Kohlenstoffstudie liefert belastbare Daten über den derzeitigen Stand und die zukünftige Entwicklung der Kohlenstoffspeicherung in den Bestandesvorräten und in den Böden der niedersächsischen Wälder und schätzt zusätzlich die darüber hinausgehenden Speicherungs- und Substitutionseffekte in Holzprodukten ab, die durch die heimische Holzwirtschaft erzeugt wurden. In dieser Hinsicht zeigt die Studie erstmalig auf Länderebene das Zusammenwirken von Forst- und Holzwirtschaft.

Die zukünftigen Ziele der niedersächsischen Kohlenstoffstudie Forst und Holz sind also die Anrechnung der $\mathrm{CO}_{2}$-Senkenleistung für die Gesamtbranche, die Substitution von konkurrierenden Bau- und Werkstoffen mit hohem Energieverbrauch durch Holz, die Aufklärung der Verbraucher bzw. das Marketing für den Bau- und Werkstoff Holz hinsichtlich einer höheren Verwendungsrate und die politische Unterstützung des Clusters Forst und Holz zur Erreichung von klimarelevanten Entscheidungen, so z. B. bei der Diskussion um einen Wald-KlimaFonds oder um ein $\mathrm{CO}_{2}$-Label für Holzprodukte. 
Holz ist schön, leicht und zugleich stark, warm und behaglich. Und es bietet eine einfache, aber effektive Möglichkeit zur Reduktion der $\mathrm{CO}_{2}$-Emissionen, die die Hauptursache des Klimawandels sind, und zwar durch

- den Kohlenstoffsenkeneffekt der Wälder,

- den Kohlenstoffspeichereffekt der Holzprodukte,

- den Ersatz für Materialien, deren Herstellung hohe $\mathrm{CO}_{2}$-Emissionen verursacht.

Diese Leistungen müssen ständig betont werden, um die Rolle des Waldes, der Forstwirtschaft, der Holzwirtschaft, der Waldeigentümer und der Menschen, die im Cluster Forst und Holz arbeiten, in der Öffentlichkeit ins richtige Licht zu setzen.

Die Kohlenstoffstudie Forst und Holz Niedersachsen leistet hierzu einen wichtigen Beitrag.
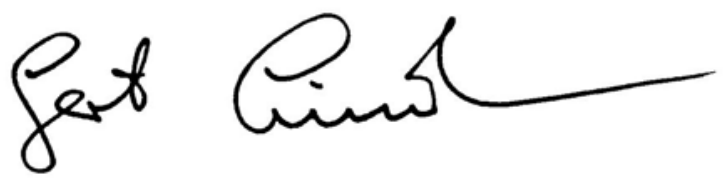

Gert Lindemann

Niedersächsischer Minister für Ernährung, Landwirtschaft, Verbraucherschutz und Landesentwicklung 


\section{Inhaltsverzeichnis}

Vorwort I

Inhaltsverzeichnis III

Zusammenfassung 1

$\begin{array}{ll}\text { Abstract } & 2\end{array}$

1 Einleitung 5

1.1 Anlass und Ziele 5

1.2 Aktuelle Daten zu CO2-Emissionen und -Senken 6

1.3 Betrachtete Speicher 7

2 Material 9

2.1 Clusterstudie Forst und Holz Niedersachsen 2007

$\begin{array}{ll}\text { 2.2 Waldböden in Niedersachsen } & 13\end{array}$

2.3 Zweite Bodenzustandserhebung (BZE II) $2008 \quad 15$

2.3.1 BZE-Netz 15

\begin{tabular}{ll} 
2.3.2 & Beprobung der BZE-Punkte \\
\hline
\end{tabular}

3 Methoden 19

3.1 Lebende Baumbiomasse 19

3.1.1 Literaturstudie zur lebenden Baumbiomasse 20

$\begin{array}{ll}3.1 .2 \text { Biomassefunktionen } & 21\end{array}$

3.1.2.1 Getestete Biomassefunktionen $\quad 21$

3.1.2.2 Auswabl geeigneter Biomassefunktionen 22

3.1.3 Berechnung der C-Vorräte der lebenden Baumbiomasse 26

$\begin{array}{ll}3.2 \text { Tote Baumbiomasse } & 27\end{array}$

3.2.1 Literaturstudie zur toten Baumbiomasse $\quad 27$

3.2.2 Berechnung der C-Vorräte der toten Baumbiomasse 28

3.3 Bodenvegetation 29

3.4 Boden 29

3.4.1 Literaturstudie zum Boden 29

3.4.2 Berechnung der C-Vorräte des Bodens 30

3.5 Holzprodukte 30

3.5.1 Literaturstudie Holzprodukte $\quad 30$

3.5.2 Kaskadennutzung 31 
3.5.3 Berechnungen zum Produktpool 32

3.5.3.1 Holaproduketklassen $\quad 32$

3.5.3.2 Holqverwendungsschlüssel 33

4 Ergebnisse $\quad 37$

4.1 Veränderungen zwischen 1987 und $2002 \quad 37$

4.2 Fortschreibung bis Ende $2006 \quad 40$

4.2.1 Lebende Baumbiomasse 40

4.2.2 Tote Baumbiomasse $\quad 42$

4.2.3 Nutzungen 42

4.2.4 Produktpool 43

4.3 Boden $2008 \quad 44$

4.3.1 Mineralböden 44

4.3.2 Moore und anmoorige Böden $\quad 45$

4.4 Simulation von 2007 bis 2036 - Szenarienvergleich 45

4.4.1 Entwicklung der Derbholzvorräte, der lebenden Baumbiomassen und deren C-Vorräte $\quad 45$

4.4.2 Nutzungen $\quad 52$

4.4.3 Tote Baumbiomasse $\quad 53$

4.4.4 Produktpool 56

5 Diskussion $\quad 61$

5.1 Material und Methoden 61

5.2 C-Speicher 2002 und Ende $2006 \quad 62$

5.2.1 Lebende Baumbiomasse $\quad 62$

5.2.2 Tote Baumbiomasse $\quad 64$

5.2.3 Mineralboden und Bodenvegetation 64

$\begin{array}{ll}5.3 \text { Szenarienvergleich } & 65\end{array}$

5.4 Carbon Forestry Management 72

6 Schlussfolgerungen und Ausblick $\quad 77$

$\begin{array}{ll}\text { Literatur } & 79\end{array}$

$\begin{array}{ll}\text { Autoren } & 90\end{array}$

$\begin{array}{ll}\text { Anhang } & 91\end{array}$ 


\title{
Kohlenstoffstudie Forst und Holz Niedersachsen
}

\author{
Forest and Wood Carbon Study, Lower Saxony
}

\section{Zusammenfassung}

Die Speicherung von Kohlenstoff im Wald ist klimapolitisch ein hochaktuelles Thema, das die niedersächsische Landesregierung mit der Kohlenstoffstudie (nachfolgend: C-Studie) aufgegriffen hat. Die Nordwestdeutsche Forstliche Versuchsanstalt (NW-FVA) wurde beauftragt, die Mengen an Kohlenstoff herzuleiten, die heute bzw. künftig in niedersächsischen Wäldern und in den aus niedersächsischem Holz hergestellten Produkten gespeichert werden. Zu diesem Zweck wurden sowohl die aktuelle lebende und tote Baumbiomasse, der Boden sowie die Holzprodukte als C-Speicher untersucht und es wurde die Entwicklung einzelner Speicher unter Zugrundelegung der drei verschiedenen Szenarien ertragsorientierter, naturnaher und naturschutzorientierter Waldbau betrachtet. Die Simulation baut auf der niedersächsischen Clusterstudie Forst und Holz (RÜTHER et al. 2007) auf und umfasst den dreißigjährigen Zeitraum von 2006 bis 2036.

Zum Zeitpunkt der ersten Bundeswaldinventur (BWI ${ }^{1}$ ) im Jahre 1987 lag der Gesamtderbholzvorrat in Niedersachsen bei rund 199 Mio. $\mathrm{m}^{3}$. Dieser Wert erhöhte sich bis zum Jahre 2002, dem Stichjahr der zweiten Bundeswaldinventur $\left(\mathrm{BWI}^{2}\right)$, auf rund $305 \mathrm{Mio} . \mathrm{m}^{3}$. Gleichzeitig stiegen die darin gespeicherten CVorräte von ca. 50 Mio. t $\mathrm{C}$ auf ca. 76 Mio. t $\mathrm{C}$ an. Die deutlichen Zunahmen sind darauf zurückzuführen, dass in der betrachteten Periode wesentlich weniger Holz eingeschlagen wurde, als nachgewachsen ist. Bis zum Beginn der Simulationsrechnungen im Jahre 2007 führten verstärkte Holzeinschläge und die Berücksichtigung der Kalamitätsnutzungen nach dem Orkan Kyrill vom 18./19.01.2007 zu einer leichten Abnahme der Gesamtderbholzvorräte auf rund 287 Mio. $\mathrm{m}^{3}$ und der 
darin gespeicherten C-Vorräte auf rund 72 Mio. t C. Unter Berücksichtigung der gegebenen Baumartenanteile, ihrer standörtlichen Zuordnung und ihres Altersaufbaus waren Ende 2006 in der lebenden Baumbiomasse der Kiefernbestände höhere C-Vorräte gespeichert (ca. 22,16 Mio.t C) als in den Buchen- (ca. 20,04 Mio. t C) oder Fichtenbeständen (ca. 18,25 Mio. t C). Am geringsten war dieser Wert in der lebenden Baumbiomasse der niedersächsischen Eichenbestände (ca. 11,53 Mio. t C). Diese Reihenfolge ändert sich aber bei einer flächenbezogenen Betrachtung. So weist die Buche die höchsten C-Vorräte pro Hektar auf $(133,1 \mathrm{t} \mathrm{C} / \mathrm{ha})$, gefolgt von Eiche (96,4 t C/ha), Fichte (86,7 t C/ha) und Kiefer $(67,7 \mathrm{tC} / \mathrm{ha})$. Die C-Vorräte im Speicher Totholz beliefen sich auf rund 2,5 Mio. t C. Die anschließenden Simulationsrechnungen zeigen, dass die Entwicklung der C-Speicher in der lebenden bzw. toten Baumbiomasse stark vom jeweils gewählten Waldbauszenario abhängt.

Der Waldboden (Humusauflage + Mineralboden bis $90 \mathrm{~cm}$ Tiefe) hat in den Laubbaumbeständen einen mindestens ebenso großen C-Vorrat wie deren lebende Baumbiomasse, während er in Nadelbaumbeständen bis zu doppelt soviel C speichern kann. Insbesondere Moore und anmoorige Böden sind wichtige CSpeicher, die erhalten werden sollten.

Holzprodukte leisten ebenfalls einen wesentlichen Beitrag für die Kohlenstoffbilanzen. Durch die stoffliche Nutzung vergrößern sie den Gesamtspeicher und substituieren Baustoffe, die in der Herstellung energieaufwendig sind. Durch die energetische Nutzung von Roh- und Altholz wird die $\mathrm{CO}_{2}$-Freisetzung durch den Einsatz fossiler Brennstoffe vermieden. Nadelholz, das überwiegend als Bauholz oder als Holzwerkstoff mit längerer Verweildauer verwendet wird, leistet gegenüber dem stärker energetisch genutzten Laubholz einen höheren Beitrag zur CSpeicherung im Produktspeicher.

Die C-Studie zeigt, dass der niedersächsische Wald und die aus ihm hervorgegangenen Holzprodukte einen wichtigen Beitrag zur C-Speicherung leisten und dass die Speichermengen durch die Strategien der Forst- und Holzwirtschaft beeinflusst werden können.

Stichworte: Kohlenstoffspeicherung, Biomasse, Boden, waldbauliche Szenarien, Holzprodukte

\begin{abstract}
The sequestration of carbon in forests is a major current political issue in the climate change debate today, which the Lower Saxony State Government has pursued through the carbon study (hereafter, C-study). The Northwest German Forest Research Station (NW-FVA) was commissioned to derive the amount of carbon stored in existing and future forests in Lower Saxony as well as in products manufactured from wood from forests in Lower Saxony. To this end, both the actual
\end{abstract}


living and dead tree biomass, the soil as well as wood products were investigated for stored carbon, and the development of various C-pools were assessed in three different scenarios adopting silvicultural systems prioritising yield, near-natural and conservation objectives. The simulation is based on the Lower Saxony Forest and Wood Cluster Study (RÜTHER et al. 2007), and covers the thirty-year period from 2006 to 2036.

At the time of the first German National Forest Inventory (BWI ${ }^{1}$ ) in 1987, the total volume of growing stock in Lower Saxony was about 199 mill. $\mathrm{m}^{3}$. In 2002, when the second inventory $\mathrm{BWI}^{2}$ was conducted, this value had increased to about 305 mill. $\mathrm{m}^{3}$. Simultaneously the carbon stored in growing stock rose from ca. 50 mill. $\mathrm{t} C$ to ca. 76 mill. t C. This marked increase resulted because the amount of wood harvested was considerably lower than that which grew during this period. Then up til 2007, at which time simulation calculations were commenced, intensification of wood harvesting and utilisation of calamity wood after hurricane Kyrill on 18./19.01.2007 lead to a slight reduction in the total volume of growing stock to about 287 mill. $\mathrm{m}^{3}$ and hence also in the sequestered carbon to round 72 mill. $t$ C. Given the existing tree species proportions, their site requirements and age structures, by the end of 2006, the carbon sequestered in the living tree biomass of Scots pine stands (ca. 22.16 mill. t C) was higher than in European beech (ca. 20.04 mill. t C) or Norway spruce stands (ca. 18.25 mill. t C). The lowest value was found in the living tree biomass in oak forests in Lower Saxony (ca. 11.53 mill. t C). The order changes, if Carbon storage per unit area is considered. European beech has the highest carbon storage per hectare $(133,1 \mathrm{t} \mathrm{C} / \mathrm{ha})$, followed by oak $(96,4 \mathrm{tC} / \mathrm{ha})$, Norway spruce $(86,7 \mathrm{tC} / \mathrm{ha})$, and Scots pine $(67,7 \mathrm{t} \mathrm{C} / \mathrm{ha})$. Sequestered carbon in deadwood amounted to about 2.5 mill. $\mathrm{t}$ C. Subsequent simulation calculations show that $\mathrm{C}$-sequestration development in the living and the dead tree biomass is strongly related to the silvicultural scenario selected.

In broadleaved stands, the forest soil (forest floor + mineral soil to $90 \mathrm{~cm}$ depth) has an equally large, if not larger, store of carbon as the living biomass, whereas, in the coniferous stands, the forest soil can sequester up to twice as much C. In particular moor and half-bog soils are important C-pools, which should be conserved.

Wood products also make an important contribution to the carbon balance. The use of wood products increases the total C-pool and replaces other construction materials that are more energy intensive to produce. By using roundwood and residual-wood for energy production, the $\mathrm{CO}_{2}$ release from fossil fuels can be avoided. Softwoods, which are predominantly used for long-lived wood products such as construction wood or in processed wood products, make a greater contribution to C-sequestration compared to hardwoods, which are more often used for energy production. 
The C-study shows that forests in Lower Saxony, and the wood products deriving from them, make an important contribution to C-sequestration, and also that this sequestration can be influenced by forest and wood managment strategies.

Keywords: carbon sequestration, biomass, soil, silvicultural management scenarios, wood products 


\section{Einleitung}

Mit dem vierten Intergovernmental Panel on Climate Change-Sachstandsbericht (IPCC 2007) hat die Klimadiskussion eine neue Qualität erreicht. Es bestehen kaum noch Zweifel, dass die globale Erwärmung bereits eingesetzt hat und maßgeblich durch die von den Menschen freigesetzten Treibhausgase verursacht wird. Eine Schlüsselstellung hat diesbezüglich der mit der Industrialisierung einsetzende exponentielle Anstieg der $\mathrm{CO}_{2}$-Konzentration in der Atmosphäre, weshalb die Vermeidung von $\mathrm{CO}_{2}$-Emissionen bzw. deren Speicherung vorrangige politische Ziele sind. Bezogen auf die Kohlenstoffspeicherung im Forstbereich kommt der wissenschaftliche Beirat der Bundesregierung Globale Umweltveränderungen zu dem Schluss: „Auf lange Sicht wird eine Strategie des nachhaltigen Forstmanagements mit der Zielrichtung, die Kohlenstoffvorräte zu erhalten oder zu erhöhen und gleichzeitig einen jährlichen Ertrag an Holzprodukten und Energie aus den Wäldern zu erhalten, den höchsten anhaltenden Nutzen für den Klimaschutz erzielen.“ (WBGU 2009). JARVIS et al. (2005) führen den hierzu passenden Begriff des Carbon Forestry Management ein, unter dem sie alle Maßnahmen verstehen, die zur Erhaltung der gespeicherten Kohlenstoffvorräte (C-Vorräte) in den Wäldern und zur Erhöhung der Senkenleistung der Wälder beitragen.

\subsection{Anlass und Ziele}

Das Thema Kohlenstoffspeicherung (C-Speicherung) ist klimapolitisch hochaktuell. Wälder stellen oft eine Senke für Kohlenstoff dar. Die Wälder der gemäßigten Klimazone speichern beispielsweise jährlich eine Menge von ca. 0,37 Mrd. t C (ROBINSON 2007). NABUURS et al. (2008) weisen aber darauf hin, dass auch europäische Wälder eine Kohlenstoffquelle (C-Quelle) sein können. Dies hängt letztendlich davon ab, ob mehr Kohlenstoff durch die Photosynthese aufgenommen oder durch die Respiration der Pflanzen, deren Ernte, Zersetzung oder durch Verbrennung freigesetzt wird. In Deutschland hat bislang ein Vorratsaufbau mit entsprechend steigender Senkenleistung stattgefunden. Nach den Ergebnissen der Inventurstudie 2008 weisen die deutschen Wälder mit $330 \mathrm{~m}^{3} /$ ha die höchsten durchschnittlichen Holzvorräte in Europa auf (OEHMicheN et al. 2011). Die Wälder in Niedersachsen leisten hierzu ihren Beitrag und tragen damit wesentlich zur C-Speicherung bei.

Ziel der niedersächsischen Landesregierung ist es, die Bedeutung des Waldes sowie der Forst- und Holzwirtschaft für den Klimaschutz stärker in das Bewusstsein der Menschen zu rücken. Sie betont in diesem Zusammenhang die Bedeutung der Wälder als Kohlenstoffsenke, die zusätzliche Kohlenstoffspeicherung in langlebigen Holzprodukten sowie die Vermeidung von Holzimporten aus Ländern, die nicht nachhaltig wirtschaften. Des Weiteren wird der Substitution von energie- 
intensiv produzierten Roh- und Baustoffen sowie von fossilen Energieträgern ein hoher Stellenwert beigemessen (ML 2010).

Nach Artikel 3.4 des Kyoto-Protokolls kann sich Deutschland die Waldbewirtschaftung auf die nationale Treibhausgasbilanz anrechnen lassen. Diese Möglichkeit nutzt die Bundesregierung seit 2006. Zur Quantifizierung der Senkenleistung ist bisher der Waldökosystemansatz verbindlich. Hierbei wird das Waldökosystem innerhalb seiner Grenzen betrachtet und jede Holznutzung einer C-Freisetzung gleichgesetzt. Im Ergebnis wird so der Holzsektor und seine Möglichkeiten, C zu speichern, bisher nicht in die Treibhausgasbilanzen einbezogen. Dies soll sich mit dem Nachfolgevertrag des Kyoto-Protokolls ändern (HEUER 2010). Damit ergibt sich auf Bundes- und Landesebene die Notwendigkeit, den Beitrag der Holzprodukte zur C-Sequestrierung besser zu quantifizieren.

Dieser Aufgabe stellt sich das Niedersächsische Ministerium für Ernährung, Landwirtschaft, Verbraucherschutz und Landesentwicklung mit der C-Studie Forst und Holz Niedersachsen. Sie soll belastbare Daten über den derzeitigen Stand und die zukünftige Entwicklung der Kohlenstoffspeicherung in den Bestandesbiomassen und Böden der niedersächsischen Wälder liefern und darüber hinaus die zusätzlichen Speicherungs- und Substitutionseffekte in Holzprodukten abschätzen.

\subsection{Aktuelle Daten zu $\mathrm{CO}_{2}$-Emissionen und -Senken}

Deutschland verursacht als hoch technisiertes Land hohe Treibhausgasemissionen mit einem Anteil von rund $87 \% \mathrm{CO}_{2}$. Im Jahr 2008 wurden deutschlandweit Treibhausgase in Höhe von 959 Mio. t $\mathrm{CO}_{2}$ - ̈̈quivalenten (davon 833 Mio. t CO 2 ) freigesetzt, davon $76 \%$ durch den Verkehr und den Energieverbrauch von Haushalten und Industrie (UMWELTBUNDESAMT 2010a). Für das Jahr 2009 geht das Umweltbundesamt in einer ersten Schätzung von 878 Mio. t $\mathrm{CO}_{2}$ - ̈̈quivalenten (davon 765 Mio. $\mathrm{CO}_{2}$ ) aus, was eine deutliche Verringerung der Emissionen darstellt. Dieser Trend wird seit 1990 festgestellt. Dennoch verursacht jeder Bundesbürger einen Pro-Kopf-Ausstoß von rund 11 t $\mathrm{CO}_{2}$ - ̈̈quivalenten pro Jahr. Davon entfallen $25 \%$ der Treibhausgasemissionen auf den Bereich Heizung und Strom, $23 \%$ auf Mobilität, $14 \%$ auf Ernährung, $28 \%$ auf den restlichen Konsum und $10 \%$ auf die öffentliche Infrastruktur (UMWELTBUNDESAMT 2010b).

Den Emissionen der mobilen und stationären Verbrennung steht das Ökosystem Wald als eine $\mathrm{CO}_{2}$-Senke gegenüber. In der Gesamtderbholzmasse waren 2002 rund 756 Mio. t C gespeichert. Im Jahr 2008 waren bundesweit in der lebenden Biomasse der Waldbäume 1,23 Mrd. t C gespeichert, was einem C-Vorrat von $120 \mathrm{t} \mathrm{C} /$ ha entspricht. Laut BWI ${ }^{2}$ beträgt der Flächenanteil der Nadelbäume in Deutschland knapp $58 \%$, der der Laubbäume $40 \%$ (Rest: Lücke/Blöße). Bei Betrachtung der Derbholzvorräte verschiebt sich diese Relation zugunsten der Nadelbäume. Deren Anteil am Gesamtvorrat beläuft sich auf $64 \%$, derjenige der Laubbäume auf $36 \%$. Aufgrund dieser höheren Vorräte und trotz der geringeren 
Rohdichte akkumulieren die Nadelbäume $57 \%$ des C-Vorrates, während $43 \%$ auf die Laubbäume entfallen. Im Totholz $(\mathrm{d} \geq 10 \mathrm{~cm})$ waren insgesamt 35 Mio. $\mathrm{t} C$ gespeichert, was im Mittel 3,25 t C/ha entspricht. Aus den Vorratsänderungen zwischen den beiden bisher durchgeführten Bundeswaldinventuren (BWI1 1987 und BWI2 2002) in den alten Bundesländern bzw. zwischen den Erhebungen für den Datenspeicher Waldfond und der zweiten Bundeswaldinventur in den neuen Bundesländern konnte für die lebende Baumbiomasse in deutschen Wäldern eine Senkenwirkung von 17 Mio. t C pro Jahr festgestellt werden. Dabei lag die Senkenwirkung in den neuen Bundesländern (2,52 t C/ha*a) höher als in den alten Bundesländern (1,22 t C/ha*a). Durch die Inventurstudie 2008 konnte für den Zeitraum von 2002 bis 2008 erneut eine Steigerung der Senkenwirkung von 4,7 Mio. t C pro Jahr (rund 0,4 t C/ha*a) nachgewiesen werden (DUNGER et al. 2009).

\subsection{Betrachtete Speicher}

In dieser Studie werden die folgenden Kohlenstoffspeicher im Ökosystem Wald berücksichtigt bzw. deren Entwicklung unter verschiedenen Behandlungsszenarien betrachtet (s. Abb. 1):

- lebende Baumbiomasse

- die gesamte oberirdische Biomasse der Bäume wie Stämme, Äste, Rinde, Stümpfe, Samen und das Blattwerk

- unterirdisch: die lebenden Grobwurzeln, jedoch nicht die Feinwurzeln $(\mathrm{d}<2 \mathrm{~mm})$

- tote Baumbiomasse

- das oberirdische tote Derbholz ab $7 \mathrm{~cm}$ Durchmesser

- Bodenvegetation

- die Vegetationsschicht aus Gräsern und Kräutern

- Mineralboden mit organischer Auflage

- Auflage und Mineralboden bis zu einer Tiefe von $90 \mathrm{~cm}$. Auch die Feinwurzeln zählen zu diesem Pool, da sie bei der Analyse von Bodenproben nach der Trocknung nicht vom Boden zu trennen sind.

- Holzprodukte

- Produkte aus Holz wie Bauholz, Holzwerkstoffe, Papier und Pappe bis hin zu Brennholz

Der Speicher Holzprodukte ist kein direkter Speicher des Ökosystems Wald. Da aber in Holzprodukten ebenfalls $\mathrm{C}$ gebunden wird, das den waldbezogenen Gesamtspeicher vergrößert, werden hierzu auch Speicherleistungen berechnet. Es ist zu erwarten, dass sie eine wichtige Rolle spielen werden, wenn die zu erwartenden Änderungen in der Anrechnung der Waldbewirtschaftung nach dem Kyoto- 
Protokoll bzw. dessen Nachfolgevereinbarung zum Tragen kommen (HEUER 2010).

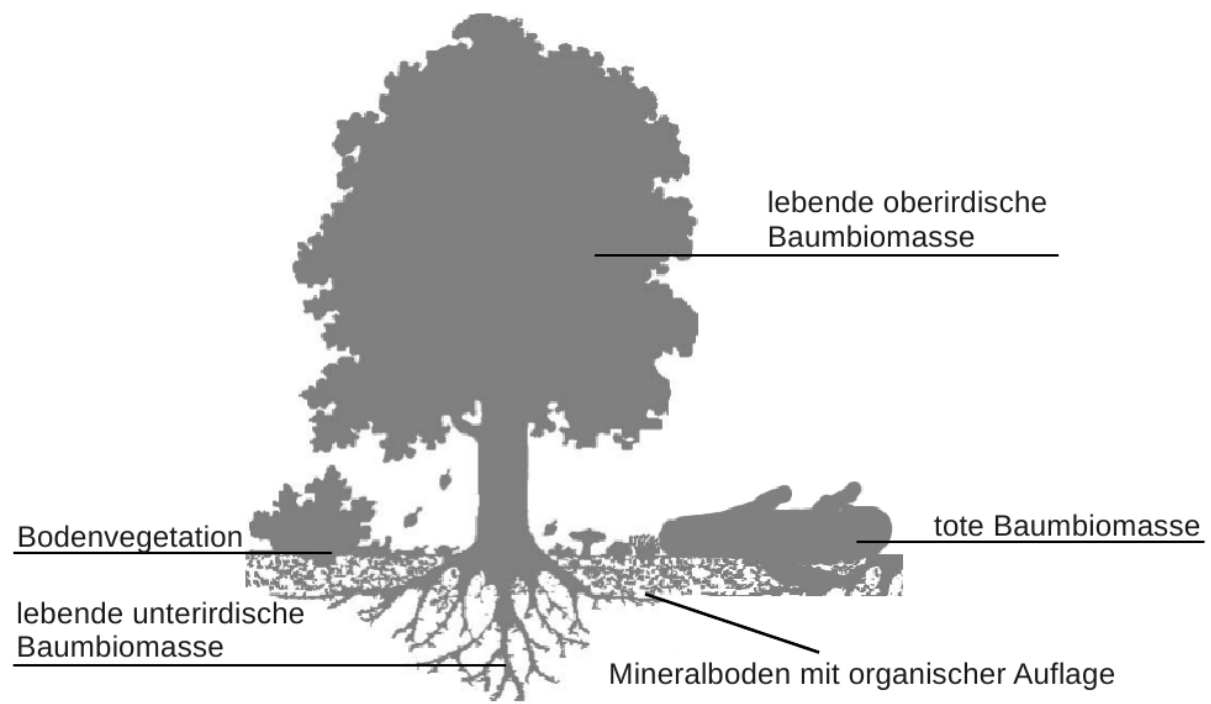

Abbildung 1: Betrachtete C-Speicher im Waldökosystem (verändert nach WBGU (1998)) 


\section{Material}

Die Ergebnisse der Clusterstudie Forst und Holz Niedersachsen und der zweiten Bodenzustandserhebung (BZE II) bilden überwiegend die Eingangsgrößen zur Berechnung der C-Vorräte der betrachteten Speicher mit verschiedenen Funktionen und Methoden. Es wird im Folgenden auf die Generierung der Daten der Clusterstudie Forst und Holz Niedersachsen und deren Ergebnisse (RÜTHER et al. 2007) kurz eingegangen, eine Beschreibung der Böden in Niedersachsen gegeben und die BZE II in Niedersachsen vorgestellt. Danach werden die einzelnen Speicher betrachtet und die jeweilige Vorgehensweise zur Berechnung der C-Vorräte beschrieben.

\subsection{Clusterstudie Forst und Holz Niedersachsen 2007}

Die Clusterstudie Forst und Holz Niedersachsen zeigt die Verflechtungen innerhalb der Forst- und Holzwirtschaft in Niedersachsen auf. Sie prognostiziert das zukünftige Aufkommen an Rohholz und schafft somit eine Diskussionsgrundlage zwischen Politik, Wirtschaft und Wissenschaft zur Bedeutung, den Aussichten und Chancen der Branche. Es wird der niedersächsische Cluster Forst und Holz mit Hilfe der Jahresumsätze, Unternehmens- und Beschäftigungszahlen sowie mit den regionalen und überregionalen Rohholzströmen analysiert. Ein weiteres Augenmerk wird auf die Untersuchung des Nutzungsverhaltens zwischen den beiden Bundeswaldinventuren gelegt. Die Betrachtung der forstlichen Nutzungspotenziale der Baumarten Buche, Eiche, Fichte und Kiefer und ihre Entwicklung bis zum Jahre 2036 unter Annahme verschiedener waldbaulicher Konzepte ist ein weiterer Schwerpunkt, auf dem die vorliegende C-Studie vor allem aufbaut (RÜTHER et al. 2007). Einige Ergebnisse hierzu sollen im Folgenden kurz vorgestellt werden.

Nach den Ergebnissen der BWI ${ }^{2}$ ist die Waldfläche Niedersachsens die drittgrößte in Deutschland. Sie beträgt 1.155 .737 ha, was einem Waldflächenanteil von 24,3 \% entspricht. Niedersachsen ist durch einen hohen Privatwaldanteil von $59 \%$ gekennzeichnet. Der Bundes- und Körperschaftswald nimmt einen geringen Anteil an der niedersächsischen Gesamtwaldfläche ein, der Anteil des Landeswaldes beträgt rund ein Drittel (s. Abb. 2, links). Die führenden Hauptbaumarten sind Kiefer (Pinus sylvestris L.), Fichte (Picea abies [L.] Karst.), Buche (Fagus sylvatica L.) und Eiche (Quercus spec. L.) mit einem Anteil von 30\%, $20 \%, 14 \%$ und $11 \%$ an der Waldfläche bzw. $27 \%, 23 \%, 18 \%$ und $12 \%$ am Holzvorrat. Der Gesamtholzvorrat beträgt $296 \mathrm{Mio}$. Vfm und setzt sich aus $43 \%$ Laub- und $57 \%$ Nadelholz zusammen. Dabei nehmen Laubhölzer mit niedriger Lebensdauer (ALn) einen hohen Anteil von $10 \%$ am Vorrat ein (s. Abb. 2, rechts bis Abb. 4) (ML 2004).

Beiträge aus der NW-FVA, Band 6, 2011 

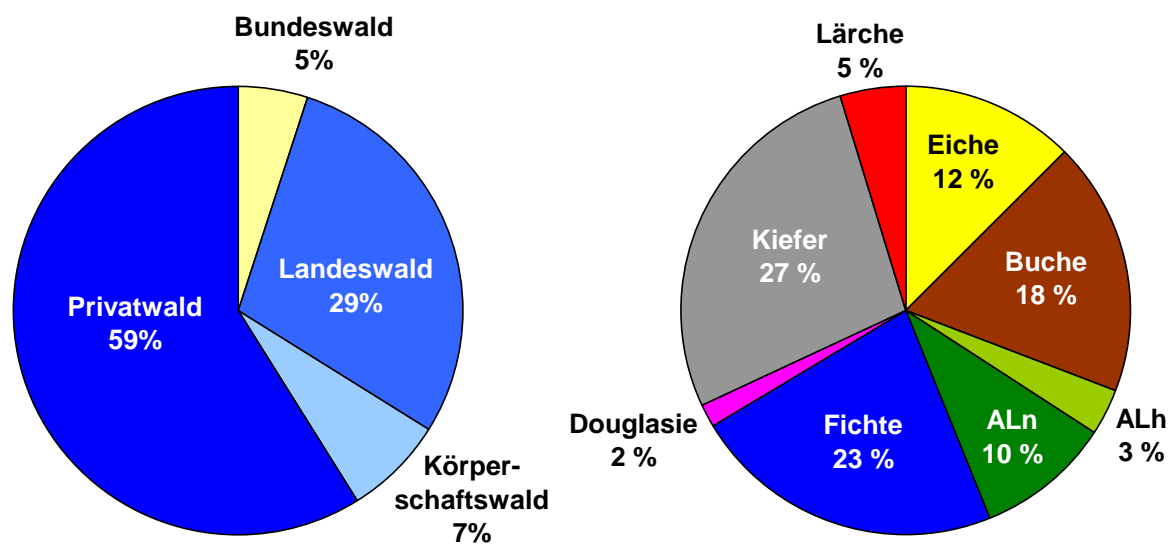

Abbildung 2: Waldflächenanteile nach Eigentumsarten (links) und Baumartenanteile am Holzvorrat in Niedersachsen (rechts) auf Basis der BWI

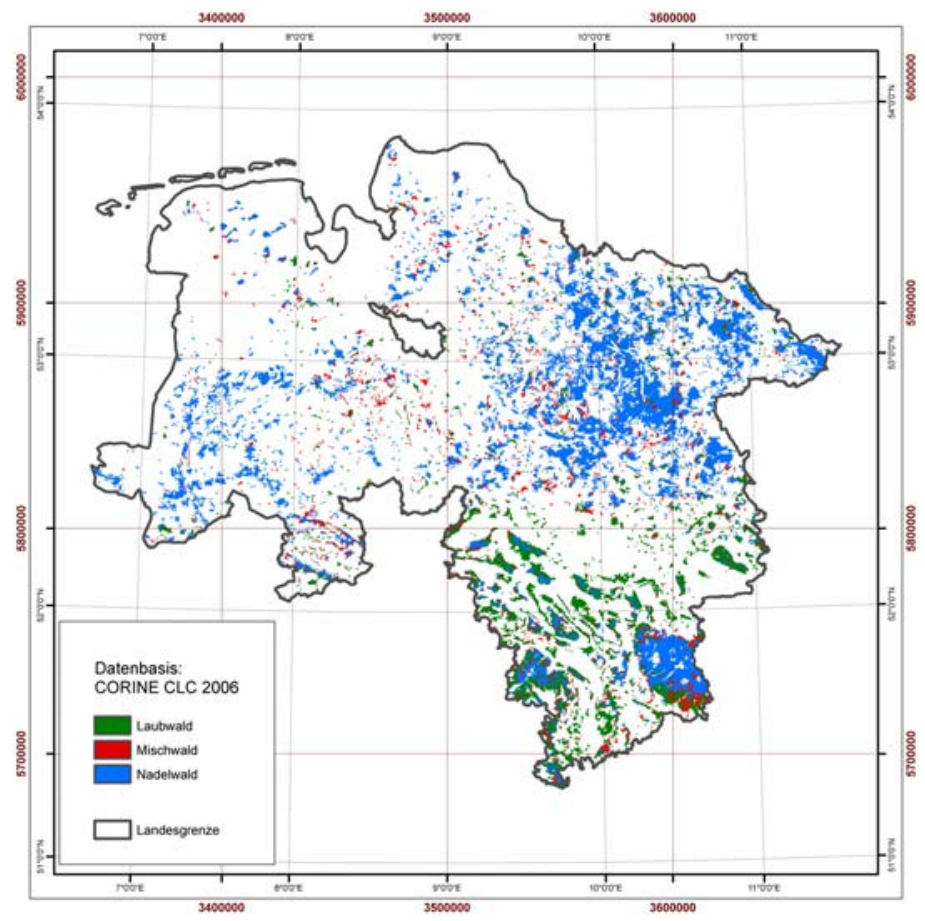

Abbildung 3: Verteilung der Laub-, Nadel- und Mischwälder in Niedersachsen auf Basis der Corine Landcover-Daten 2006 


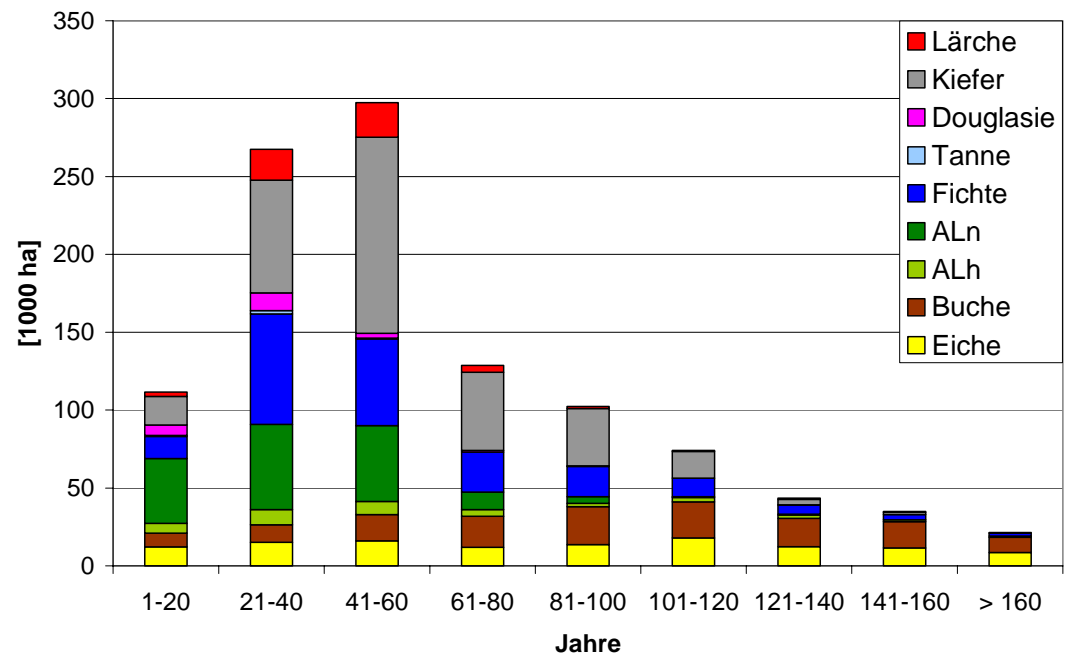

Abbildung 4: Flächenanteile der Baumartengruppen im Hauptbestand nach Altersklassen in Niedersachsen auf Basis der BWI

Die Berechnungen und Analysen der C-Speicherung in der lebenden und toten Baumbiomasse sowie in den Holzprodukten beruhen auf den Ergebnissen der Potenzialbetrachtungen der Clusterstudie Forst und Holz Niedersachsen (RÜTHER et al. 2007). Bei der Clusterstudie wurden die niedersächsischen Einzelbaumdaten der zweiten Bundeswaldinventur (BWI2), die Holzeinschlagsstatistik und die Sturmschadenserhebungen nach dem Orkan Kyrill zur Fortschreibung der BWI ${ }^{2}$ Daten auf den Stichtag 31.12.2006 herangezogen. Diese Werte wurden anschließend genutzt, um mit dem Programmsystem WaldPlaner die weitere Waldentwicklung getrennt für die Szenarien ertragsorientierter Waldbau, naturnaher Waldbau und naturschutzorientierter Waldbau für 30 Jahre zu simulieren (s. Abb. 5).

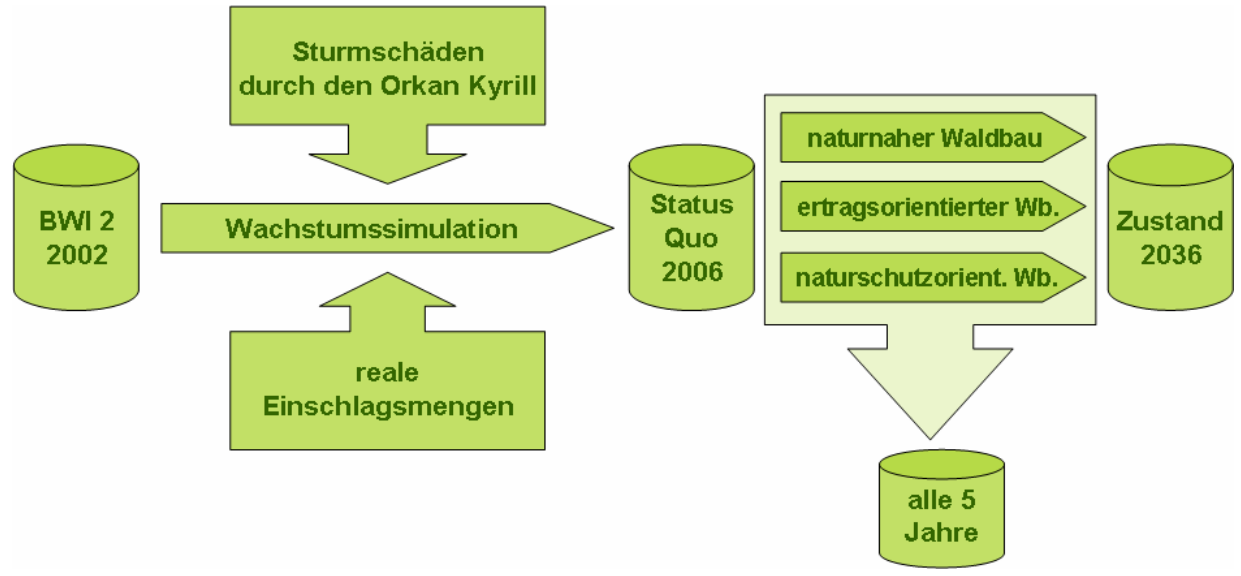

Abbildung 5: Schematischer Aufbau des Gesamtprognosesystems (RÜTHER et al. 2007)

Beiträge aus der NW-FVA, Band 6, 2011 
Die Steuerung der Szenarien erfolgte über naturschutz- und eingriffsspezifische Parameter, deren Kombinationen einen weiten Bereich der möglichen Waldbaumaßnahmen abdecken (s. Tab. 1). Es werden dabei sowohl unterschiedliche Naturschutz- als auch Durchforstungs- und Endnutzungmaßnahmen umgesetzt.

Tabelle 1: $\quad$ Rabmenwerte der drei gerechneten Szenarien der Clusterstudie Forst und Holz Niedersachsen (RÜTHER et al. 2007)

\begin{tabular}{|c|c|c|c|}
\hline Parameter & ertragsorientiert & naturnah & $\begin{array}{l}\text { naturschutz- } \\
\text { orientiert }\end{array}$ \\
\hline Schutz seltener Baumarten & nein & ja & ja \\
\hline Habitatbäume (n/ha) & 0 & 3 & 10 \\
\hline Totholz (m³/ha) & bis 10 & 20 & 40 \\
\hline Zielstärke (cm) & $\begin{array}{l}\text { Ei } 65, \mathrm{Bu} 55, \\
\text { Fi 40, Ki } 40\end{array}$ & $\begin{array}{l}\text { Ei 70, Bu 60, } \\
\text { Fi 45, Ki 45 }\end{array}$ & $\begin{array}{l}\text { Ei } 75, \mathrm{Bu} 65, \\
\text { Fi } 50, \mathrm{Ki} 50\end{array}$ \\
\hline $\begin{array}{l}\text { Endnutzungsmasse pro } \\
\text { Hieb }\left(\mathrm{m}^{3} / \mathrm{ha}\right)\end{array}$ & $\begin{array}{l}\text { Min. } 10-15 \\
\text { Max. } 70-115\end{array}$ & $\begin{array}{l}\text { Min. } 10-15 \\
\text { Max. } 70-115\end{array}$ & $\begin{array}{l}\text { Min. } 10-15 \\
\text { Max. } 60-120\end{array}$ \\
\hline $\begin{array}{l}\text { Durchforstungsbeginn } \\
\text { Bestandesoberhöhe (m) }\end{array}$ & $12-18$ & $10-16$ & $10-16$ \\
\hline $\begin{array}{l}\text { Durchforstungsmasse pro } \\
\text { Hieb }\left(\mathrm{m}^{3} / \mathrm{ha}\right)\end{array}$ & $25-110$ & $25-100$ & $25-110$ \\
\hline $\begin{array}{l}\text { Freistellungsgrad der Z- } \\
\text { Bäume }\end{array}$ & sehr stark & stark & stark \\
\hline
\end{tabular}

Seltene Baumarten genießen als Minderheiten bei dem naturschutzorientierten und naturnahen Waldbau besonderen Schutz. Auch Habitatbäume als Lebensraum für viele Organismen werden in unterschiedlichem Umfang der Nutzung entzogen. Weiterhin sind die angestrebten Totholzvorräte eine wichtige Steuerungsgröße. Sie liegen zwischen $10 \mathrm{~m}^{3} /$ ha beim ertragsorientierten und $40 \mathrm{~m}^{3} /$ ha beim naturschutzorientierten Szenario. Der Endnutzungszeitpunkt wird durch die Zielstärke baumartenspezifisch gesteuert und variiert, ausgehend vom Szenario naturnaher Waldbau, um jeweils - $5 \mathrm{~cm}$ beim ertragsorientierten Waldbau und jeweils $+5 \mathrm{~cm}$ beim naturschutzorientierten Waldbau für die untersuchten Baumarten. Die Zielstärken liegen z. B. für Eiche zwischen 65 und $75 \mathrm{~cm}$ BHD oder bei Kiefer zwischen 40 und $50 \mathrm{~cm}$ BHD. Der Durchforstungsbeginn wird über die Bestandesoberhöhe festgelegt. Er liegt bei der ertragsorientierten Variante im Mittel um $2 \mathrm{~m}$ höher als bei den anderen Varianten. Beim ertragsorientierten Szenario wird damit später aber gleichzeitig stärker durchforstet. In den Endnutzungsmassen pro Hieb unterscheiden sich die Szenarien nur wenig, da deren Höhe von den Flächen und Risiken bestimmt wird. Für weitere Erläuterungen siehe RÜTHER et al. (2007). 


\subsection{Waldböden in Niedersachsen}

Die Waldböden in Niedersachsen sind sehr heterogen. Wald stockt zumeist auf Flächen, die sich in der Vergangenheit weniger gut für die landwirtschaftliche Nutzung eigneten, weil die Witterung zu ungünstig, das Gelände zu steil, der Boden zu steinig, nass oder nährstoffarm war. Großflächig wird in Niedersachsen zwischen dem nördlichen Tiefland und südlichen Bergland unterschieden. Das Tiefland teilt sich in das Höhen- und das Talpleistozän sowie das Holozän auf. Das Höhenpleistozän besteht aus den Grundmoränen, Endmoränen und Sanderflächen. Hier finden sich vor allem Geschiebelehme, ärmere und mittlere, teilweise auch reichere Sande, Sandlöss und Lauenburger Ton als Hauptsubstrate. Im Allgemeinen haben diese Standorte keinen Grundwasseranschluss, eventueller Wasserüberschuss ist allenfalls durch Stauwasser bedingt. Im Talpleistozän und Holozän sind Talsande und Auenstandorte, Moore und Brücher die wesentlichen standörtlichen Bodeneinheiten; diese Standorte sind mehr oder weniger stark durch Grundwasser beeinflusst (ОTTO 1972). Im Übergang zum Bergland liegt die stark lössbeeinflusste, flach wellige Berglandschwelle, die überwiegend landwirtschaftlich genutzt wird. Das geologisch vielgestaltige Bergland mit Gesteinen aus Trias, Jura und Kreide sowie dem Niedersächsischen Harz mit alten Gesteinen aus dem Paläozoikum ist bodenkundlich außerordentlich vielgestaltig und die Standorte wechseln - auch in Folge des Reliefs - auf kleinstem Raum. Häufig finden sich Lössfließerden über Buntsandstein, auch Muschelkalk und mächtige Lössdecken sind weit verbreitet. Es überwiegen schluffig-lehmige bis sandige, basenarme Silikatverwitterungsböden

Eine etwas detailliertere Beschreibung der Waldböden ist auf Grundlage der acht Waldbauregionen in Niedersachsen möglich (NFP 2004), die aus unterschiedlichen Wuchsbezirken zusammengesetzt sind (s. Abb. 6). Diese unterscheiden sich vorwiegend durch die klimatischen Verhältnisse, welche ebenfalls die Bodenentwicklung beeinflussen.

Die Waldbauregion 1 besteht aus den Wuchsbezirken Bramwald-Brackenberg, Unterer und Hoher Solling sowie dem Kaufunger Wald. Geologisch ist diese Region überwiegend von Buntsandstein geprägt, teilweise findet sich auch Muschelkalk oder Basalt im Brackenberg, der großflächig durch lössgeprägte Decken überlagert wird. Die dortigen Böden sind mäßig bis ziemlich gut versorgte podsolige Braun- und Parabraunerden, in einigen Plateaulagen sind auch Pseudound Stagnogleye zu finden. Auf den Basalt- und Kalkstandorten haben sich basenreiche Braunerden und Rendzinen ausgebildet.

Bei der Waldbauregion 2 handelt es sich um das Hügelland zwischen Solling und Harz. Dort finden sich Gesteine aus dem Zeitalter des Trias (Muschelkalk, Buntsandstein, seltener Keuper) mit flächiger Überlagerung durch unterschiedlich mächtige lössgeprägte Decken. Die Böden sind überwiegend ziemlich gut bis sehr gut nähstoffversorgte Braunerden, Parabraunerden, Rendzinen und Pelosole, 
wobei auf basenarmen Buntsandsteinstandorten auch saure und teilweise podsolige Braunerden $\mathrm{zu}$ finden sind.

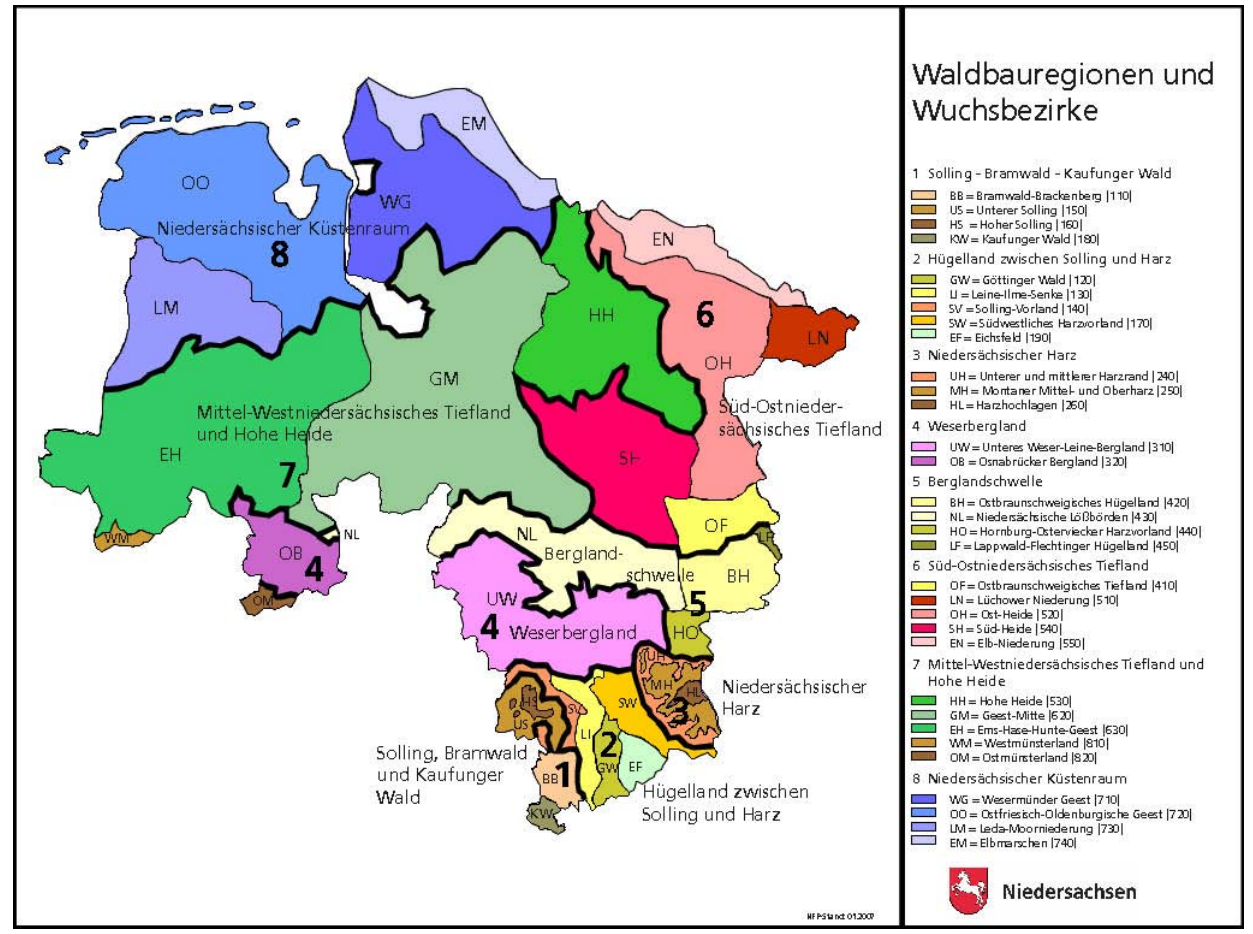

Abbildung 6: Waldbauregionen und Wuchsbezirke in Niedersachsen (NFP 2004)

Die dritte Waldbauregion umfasst den niedersächsischen Teil des Harzes, welcher 20 \% der Landeswaldfläche ausmacht. Der Harz wird ausschließlich von Gesteinen des Erdaltertums in großer Diversität geprägt, vor allem durch basenarme Grauwacken und Quarzite, aber auch durch basenreiche Gesteine wie z. B. Gips, Diabas und teilweise Tonschiefer. Die Bodenverhältnisse im Harz wechseln bedingt durch die Topographie und das Ausgangsgestein sehr kleinräumig. Die Standorte sind überwiegend mäßig bis schwach nährstoffversorgt und zumeist mindestens frisch. Hinsichtlich der Bodenbildung überwiegen basenarme Braunerden, in den Harzhochlagen gibt es aber auch Podsol-Braunerden und Podsole.

Die Waldbauregion 4, das Weserbergland, ist durch hohe Anteile von Kalkgesteinen gekennzeichnet, die auf einem Großteil der Fläche stark lössbeeinflusst sind. Auf den mäßig bis ziemlich gut versorgten Keuper- und Kreidesandsteinen herrschen teilweise podsolige Braunerden, auf den besser versorgten kalkbeeinflussten Standorten eutrophe Braunerden, Parabraunerden und Rendzinen vor. Die Buche erlangt in dieser Waldbauregion auf basenreichen mächtigen Lössdecken ihr Wuchsoptimum in Niedersachsen. 
Die fünfte Waldbauregion beschreibt die Berglandschwelle, d. h. das Ostbraunschweigische-Hügelland, die Niedersächsischen Lößbörden und das HornburgOsterviecker Harzvorland. Diese Region ist sehr gering bewaldet, daher haben die verbliebenen Wälder eine hohe ökologische Bedeutung für den überwiegend landwirtschaftlich genutzten Raum. Die Berglandschwelle ist durch die Lössbörde mit mächtigen Lösslehmen gekennzeichnet. Die Böden sind jedoch recht vielgestaltig. Es sind Braun- und Parabraunerden $\mathrm{zu}$ finden, in den staufeuchten Verebnungslagen und grundfeuchten Niederungen Braunerde-Pseudogleye und Gleye sowie Übergänge von Gleyen über Anmoor-Gleye bis zu Niedermooren auf den nassen Standorten.

Das zur Waldbauregion 6 zusammengeführte Süd-Ostniedersächsische Tiefland vereint sechs Wuchsbezirke und umfasst absolut betrachtet die zweitgrößte Waldfläche der niedersächsischen Waldbauregionen. Die Wuchsbezirke Südheide, Lüchower Niederung und Elbniederung sind von Gleyen, Podsol-Gleyen und Anmoor-Gleyen bestimmt, jedoch gibt es oft auch kleinflächige, gut versorgte Auenablagerungen. In der Ostheide überwiegen die mäßig bis ziemlich gut versorgten, grundwasserfreien Geschiebesande. Im Ostbraunschweigischen Flachland sind Pseudogleye vorherrschend. Ein großer Teil dieses Gebietes (70\%) ist nur mäßig bis sehr schwach mit Nährstoffen versorgt.

Die Waldbauregion 7, das Mittel-Westniedersächsische Tiefland und die Hohe Heide, wird geologisch im Westen durch erdgeschichtlich ältere, in der Lüneburger Heide durch jüngere Sande geprägt. Auf den schwach bis mäßig nährstoffversorgten Böden sind Podsole und Braunerde-Podsole zu finden, auf den silikatreicheren Sanden auch Braunerden. Auf den stark vom Grundwasser beeinflussten Mineralböden finden sich jedoch auch Gleye in unterschiedlichen Übergangsformen.

Der Niedersächsische Küstenraum bildet die sehr gering bewaldete Waldbauregion 8. Die dort vorherrschenden Bodentypen sind Podsole mit Übergängen zu Gleyen, seltener Braunerden. Die früher intakten Hochmoore waren in dieser Region überwiegend waldfrei.

\subsection{Zweite Bodenzustandserhebung (BZE II) 2008}

\subsubsection{BZE-Netr,}

Um den Kenntnisstand über die Dynamik der Waldböden zu verbessern, fand in der Zeit von 2007 bis 2009 in den niedersächsischen Wäldern die zweite bundesweite Bodenzustandserhebung (BZE II) statt. Sie folgte als Wiederholungsinventur der ersten Waldbodenzustandserhebung (BZE I), die in Niedersachsen in den Jahren 1990 und 1991 durchgeführt wurde. Das BZE II-Raster in Niedersachsen besteht aus einem einheitlichen 8 x 8 km-Netz mit insgesamt 169 Punkten, die die Waldflächenverteilung in Niedersachsen relativ gut repräsentieren (s. Abb. 7).

Beiträge aus der NW-FVA, Band 6, 2011 

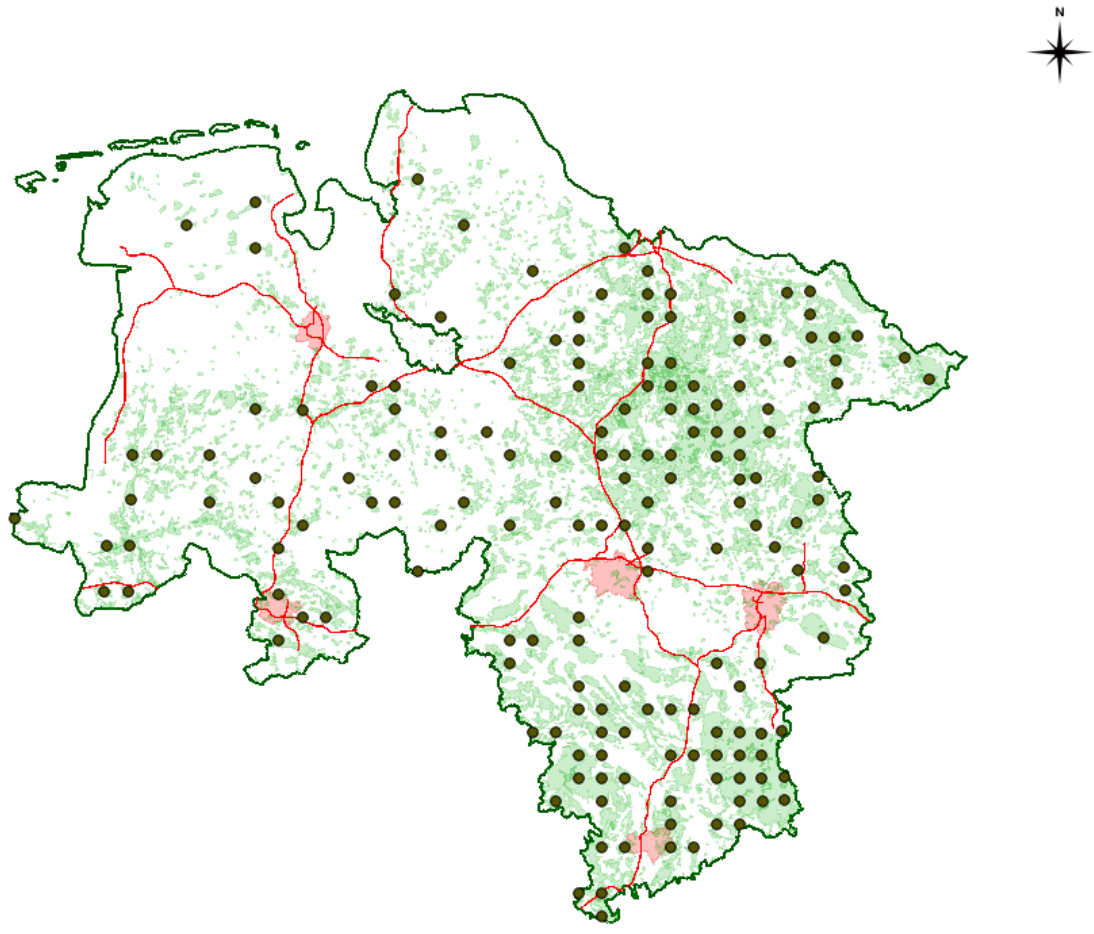

(C) 2011 SG NW-FVA D2

Abbildung 7: Verteilung der BZE II-Punkte in Niedersachsen

Die BZE II orientiert sich an dem seit 1984 bestehenden Netz der Waldzustandserhebung und nutzt die standörtliche Grundinformation sowie die langjährigen Zeitreihen der Kronenverlichtung für die Bewertungen. An jedem Punkt der BZE II wurden Auflagenhumus und Mineralboden beschrieben und beprobt, wobei in Abhängigkeit vom aufzunehmenden Parameter entweder das Profil oder 8 Satelliten um das Profil genutzt wurden. An ausgewählten Stichprobenbäumen wurden Nadel- bzw. Blattproben aus den Baumkronen zur Abschätzung des Ernährungsstatus gewonnen. Weiterhin wurden leicht versetzt zu den Bodeneinschlägen die Bestände in konzentrischen Probekreisen ertragskundlich aufgenommen, Bohrkerne für Zuwachsmessungen bei den Hauptbaumarten entnommen sowie Vegetationserhebungen durchgeführt (s. Abb. 8). Das Verfahren der BZE II ist ausführlich in der bundesweit gültigen Arbeitsanleitung beschrieben (WELLBROCK et al. 2006). 


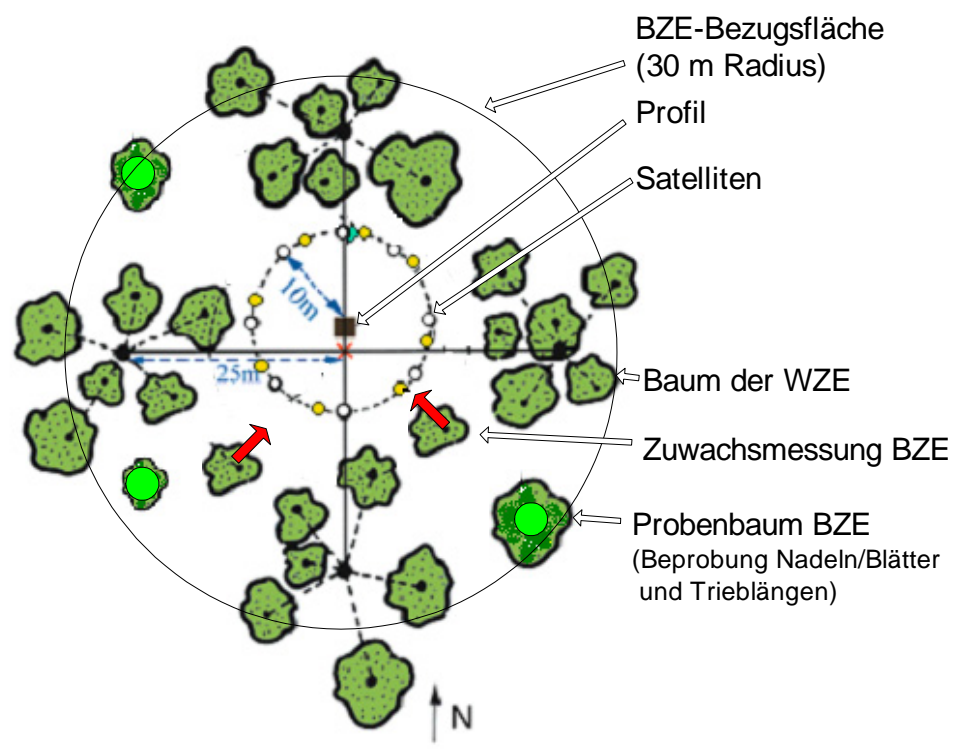

Abbildung 8: Schematische Darstellung des Aufnabmeverfahrens der BZE II

\subsubsection{Beprobung der BZE-Punkte}

Die Pedogenese und die hohe Variabilität der Bodenschichten innerhalb der Mineralböden machen es problematisch, den C-Vorrat zu ermitteln. Außerdem kann es bei einer Probennahme mit Bohrgeräten zu einer Verschleppung von Kohlenstoff durch Humusteilchen innerhalb des Bohrkerns in tiefere Bodenschichten kommen und damit zu einer Überschätzung des C in tieferen Schichten. Darüber hinaus ist es an bestimmten Standorten schwierig, den Auflagehumus vom Mineralboden zu trennen. In der Regel kommt es allein schon durch die Tätigkeit der Bodenlebewesen zu einer innigen Durchmischung von Mineralboden und Auflagehumus. Bei der Probennahme wird dieser Schwierigkeit durch eine durchgehende Bohrung mit einem volumenstarken Bohrer begegnet und erst anschließend werden Auflagehumus und Mineralboden getrennt, sodass in jedem Fall der gesamte Kohlenstoff erfasst wird, selbst wenn die Trennung von Auflage und Mineralboden nicht immer ganz exakt durchgeführt werden kann. Dem Problem der teilweise hohen kleinräumlichen Heterogenität wurde mit der Bildung von Mischproben aus 8 Einzelbeprobungen an Satellitenpunkten begegnet.

Für die Berechnung der C-Vorräte ist zunächst die analytische Bestimmung der C-Konzentration im Mineralboden und im Auflagehumus erforderlich. Dabei muss vom Gesamtvorrat der mineralische $\mathrm{C}$ (Kohlenstoff im Carbonat enthalten) abgezogen werden. Die analytischen Methoden sind in KÖNIG und FORTMANN (1996) und KÖNIG et al. (2009) beschrieben. 
Neben der Konzentration des C ist für die Bestimmung der C-Vorräte die Kenntnis der Trockenrohdichte des Feinbodens (Korngröße der Bodenpartikel $<2 \mathrm{~mm}$ ), des Skelettgehalts der jeweiligen Tiefenstufen im Mineralboden und des volumengerecht beprobten Trockengewichts des Auflagehumus erforderlich. Die Trockenrohdichte des Feinbodens wurde bis in $30 \mathrm{~cm}$ Bodentiefe an den Satelliten, ab $30 \mathrm{~cm}$ Bodentiefe am Profil analytisch über Stechzylinder bzw. in skelettreichen Böden des Berglandes über Stechkappen bestimmt. Der Skelettgehalt wurde für jede Tiefenstufe berechnet, indem im Labor die am Profil mittels einer Spaten-/ Schippenprobe gewonnene jeweilige Probe gewogen und ihre Steindichte ermittelt wurde. Die volumengerechte Mischprobe des Auflagehumus wurde im Labor getrocknet und ausgewogen. Der C-Vorrat des Auflagehumus ergibt sich als Produkt der C-Konzentration und des Trockengewichtes; der C-Vorrat des Mineralbodens wird berechnet als das Produkt der Konzentration, der Schichtmächtigkeit, der Trockenrohdichte des Feinbodens sowie des Feinbodenanteils.

Aufgrund der Beprobungsproblematik und der Diversität der Waldböden ist die flächenmäßige Bestimmung des C-Vorrates für den Speicher „Boden“ mit großen Unsicherheiten behaftet. Als Beispiele werden im Folgenden zwei Profile der BZE II gezeigt, die sehr unterschiedliche, aber überdurchschnittlich hohe CVorräte haben, um eine Vorstellung von der Variabilität der C-Vorräte zu bekommen. Die Abbildung 9 zeigt links einen Podsol nordöstlich von Schwanewede auf einem nährstoffarmen Standort, aber mit einem C-Vorrat von $260 \mathrm{t} \mathrm{C/ha} \mathrm{bis}$ $90 \mathrm{~cm}$ Tiefe. Rechts ist eine Terra fusca-Rendzina aus dem niedersächsischen Bergland zu sehen, welche in den ersten $90 \mathrm{~cm}$ Boden $140 \mathrm{t} \mathrm{C} /$ ha gespeichert hat.
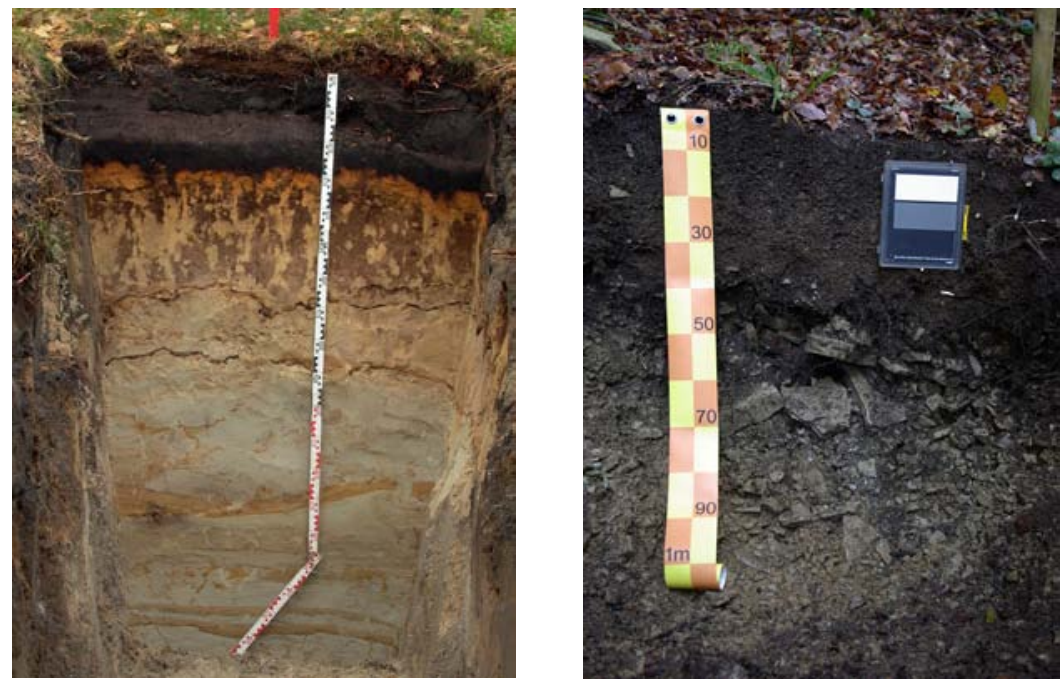

Abbildung 9: Links: Ein Podsol nordöstlich von Schwanewede mit Feinsand als Bodenart und rund 290 tC/ha bis $90 \mathrm{~cm}$ Tiefe. Rechts: Eine Terra fusca-Rendzina im niedersächsischen Bergland mit einem C-Vorrat von 140 tC/ ha bis $90 \mathrm{~cm}$ Tiefe. 


\section{Methoden}

\subsection{Lebende Baumbiomasse}

Holz besteht aus organischen Verbindungen, die wiederum aus den chemischen Elementen Kohlenstoff $(\mathrm{C})$, Wasserstoff $(\mathrm{H})$, Sauerstoff $(\mathrm{O})$ und Stickstoff $(\mathrm{N})$ aufgebaut sind. Darrtrockenes Holz (0\% Holzfeuchte) besteht zu etwa $50 \%$ aus C, zu $6 \%$ aus $\mathrm{H}$, zu $43 \%$ aus $\mathrm{O}$ und zu $1 \%$ aus $\mathrm{N}$ (KNIGGE u. SCHULZ 1966). Da im Allgemeinen die C-Konzentration zwischen den Baumarten nur wenig variiert, wird in dieser Studie auch eine C-Konzentration von $50 \%$ unterstellt (vgl. BURSCHEL et al. 1993, BAUER et al. 2000, WirTH et al. 2004a, PrETZSCH 2009, WEST 2009).

Die Umwandlung von $\mathrm{C}$ in $\mathrm{CO}_{2}$ kann man mit den absoluten Atommassen der einzelnen chemischen Elemente nachvollziehen. Sie betragen für $\mathrm{C}$ rund $12 \mathrm{~kg}$ und für $\mathrm{O}$ ca. $16 \mathrm{~kg}$. Daraus ergibt sich für $\mathrm{CO}_{2}$ eine Atommasse von $44 \mathrm{~kg}$. Das Verhältnis der Atommassen von $\mathrm{CO}_{2}$ und $\mathrm{C}$ entspricht einem Faktor von rund 3,67. Dies bedeutet, dass bei der Photosynthese aus 3,67 $\mathrm{kg} \mathrm{CO}_{2} 1 \mathrm{~kg} \mathrm{C}$ aufgebaut wird. Umgekehrt werden bei der Verbrennung von $1 \mathrm{~kg} \mathrm{C}$ etwa 3,67 $\mathrm{kg} \mathrm{CO}_{2}$ freigesetzt.

Zur Berechnung des Kohlenstoffvorrates von Wäldern ist es notwendig, die Biomasse der lebenden Bäume zu ermitteln. Generell gibt es zwei Möglichkeiten die Baumbiomasse zu schätzen: entweder werden Biomasse-Expansionsfaktoren ${ }^{1}$ (biomass factors) genutzt oder es kommen Biomassefunktionen ${ }^{2}$ (biomass equations) zum Einsatz, für die Einzelbaumdaten benötigt werden. Biomasse-Expansionsfaktoren und -funktionen stammen oftmals aus lokalen Untersuchungen und sind daher nicht repräsentativ für ganze Regionen. SOMOGYI et al. (2007) geben einen Überblick über verschiedene Biomassenexpansionsfaktoren und -funktionen und ihren Unsicherheiten sowie einen Entscheidungsbaum zur Wahl zwischen den beiden Berechnungsmöglichkeiten. In Anlehnung an diesen Entscheidungsbaum werden in dieser Arbeit Funktionen zur Schätzung der Biomasse herangezogen, da Einzelbaumdaten zur Verfügung stehen.

Für die Baumarten Kiefer, Fichte, Buche und Eiche wurden verschiedene Biomassefunktionen aus der Literatur ausgewählt und anhand der in der Nordwest-

1 Biomasse-Expansionsfaktoren sind Schätzgrößen, um vom Derbholzvorrat auf die Biomasse eines Bestandes schließen zu können. Hier werden folgende Faktoren genutzt: Reisigfaktor $=1,5$; Blattfaktor $=1,05 ;$ Nadelfaktor $=1$; Wurzelfaktor $=1,25($ PRETZSCH 2009).

2 Biomassefunktionen beschreiben die allometrischen Zusammenhänge zwischen der Veränderung der lebenden Biomasse eines Baumes und der Veränderung anderer Baummerkmale (meist Brusthöhendurchmesser und Baumhöhe). 
deutschen Forstlichen Versuchsanstalt (NW-FVA) vorhandenen Biomassedaten auf ihre Eignung überprüft (s. Kap. 3.1.2.1).

\subsubsection{Literaturstudie zur lebenden Baumbiomasse}

Die lebende Baumbiomasse wird in ober- und unterirdische Biomasse eingeteilt (s. Kap. 1.3). Der mittlere C-Vorrat in der gemäßigten Zone beläuft sich in der lebenden oberirdischen Dendromasse der Nadelbäume auf schätzungsweise $149 \mathrm{t} \mathrm{C} / \mathrm{ha}$ und in der unterirdischen Dendromasse auf $46 \mathrm{t} \mathrm{C/ha.} \mathrm{Die} \mathrm{entspre-}$ chenden Werte in Laubwäldern betragen oberirdisch ca. $109 \mathrm{t}$ C/ha bzw. unterirdisch rund $26 \mathrm{tC} /$ ha (LUYSSAERT et al. 2007). Zurzeit sind die Wälder der gemäßigten Zone C-Senken mit einer jährlichen C-Aufnahme von 0,37 Mrd.t (ROBINSON 2007). Durch menschliche Einflüsse werden die Wälder der gemäßigten Zone am stärksten beeinflusst (LORENZ u. LAL 2010) und damit auch ihre CSpeicher und C-Kreisläufe.

Für die Baumbiomasse der Wälder in Deutschland berechneten BURSCHEL et al. 1993 einen bundesweiten C-Vorrat in Höhe von 89 t C/ha auf Grundlage der BWI ${ }^{1}$ und des Datenspeichers Waldfonds. DIETER und ELSASSER (2002) kamen zu Werten von 120 bis $190 \mathrm{t} \mathrm{C/ha}$ je nach Altersklasse und Baumart basierend auf der BWI $^{1}$ und verschiedenen einzelnen Datenquellen für die neuen Bundesländer. DUNGER et al. (2009) geben auf der Basis der Inventurstudie 2008 einen Durchschnittswert von $120 \mathrm{t} \mathrm{C/ha}$ für Deutschland an. Bei diesen Zahlen handelt es sich um geschätzte Durchschnittswerte, die sehr stark in Abhängigkeit von den Standorten, den Baumarten und dem Altersaufbau der Bestände variieren können.

Die zahlreichen regionalen Untersuchungen in Deutschland zeigen trotz Unterschieden in den jeweiligen Berechnungsweisen für die einzelnen Bundesländer einen deutlichen Gradienten mit steigenden C-Vorräten von Norden nach Süden. Zudem bestehen deutliche Unterschiede in den C-Vorräten der einzelnen Baumarten. Diese erklären sich einerseits aus den baumartenspezifischen Raumdichten und Wuchsleistungen, andererseits auch durch die variierenden Standortzuordnungen, Alter und Strukturen der Bestände (vgl. z. B. BÖSWALD (1996) für Bayern, PISTORIUS (2007) für Baden-Württemberg, WIRTH et al. (2004a) für Thüringen). Für das Bundesland Niedersachsen ermittelten BÖSWALD und WIERLING (1997) auf der Basis der BWI ${ }^{1}$ insgesamt einen C-Vorrat in der lebenden Dendromasse von 71,1 Mio. t bzw. ca. 65 t C/ha.

Untersuchungen zu den einzelnen Baumarten kommen zu folgenden Ergebnissen. Die Buchenwälder in Nordrhein-Westfalen haben in ihrer oberirdischen Baumbiomasse rund $120 \mathrm{t} \mathrm{C/ha} \mathrm{gespeichert} \mathrm{(JOOSTEN} \mathrm{et} \mathrm{al.} \mathrm{2004).} \mathrm{MUND} \mathrm{(2004)}$ untersuchte den C-Vorrat in Buchenbeständen mit unterschiedlicher waldbaulicher Behandlung im Hainich-Dün-Gebiet. In Beständen mit Schirmschlagbetrieb wurden im Mittel $154 \mathrm{t} \mathrm{C/ha,} \mathrm{in} \mathrm{plenterartig} \mathrm{bewirtschafteten} \mathrm{Wäldern} 176 \mathrm{t} \mathrm{C/ha}$ und in unbewirtschafteten Wäldern des Nationalparks Hainich $247 \mathrm{t} \mathrm{C} /$ ha in der 
lebenden oberirdischen Baumbiomasse festgestellt. RADEMACHER et al. (2009) kamen in Buchenbeständen des Sollings und des Göttinger Waldes zu Gesamtbiomassevorräten von 196 bis $248 \mathrm{t} / \mathrm{ha}$, die etwa 98 bis $124 \mathrm{t} \mathrm{C} / \mathrm{ha}$ entsprechen. PELLINEN (1986) untersuchte die gesamte ober- und unterirdische Biomasse von Buchen im Göttinger Wald und ermittelte Werte in Höhe von 370 bis 430 t/ha, was in etwa einem C-Vorrat von 185 bis 215 t C/ha entspricht. In der Literaturstudie von JACOBSEN et al. (2003) wird für die Buchenbestände in Deutschland eine oberirdische Biomasse in Höhe von 347 t/ha angegeben, die etwa $174 \mathrm{t} \mathrm{C} /$ ha entspricht.

WEIS und GÖTTLEIN (2002) fanden in einem 96-jährigen Fichtenbestand in der Nähe von Augsburg eine oberirdische Biomasse von 590 t/ha, in der etwa 295 t C/ha gespeichert waren. SCHWARZMEIER (2000) berechnete oberirdische Trockenmassen in Höhe von 441 bzw. 458 t/ha für zwei bayerische Fichtenbestände (85 und 100 Jahre), die etwa 220 bzw. 229 t C/ha entsprechen. Zu etwas geringeren Werten kam ILG (2002) in einem 85-jährigen Fichtenbestand in Bayern. Die oberirdische Gesamtbaumbiomasse betrug 377 t/ha, der C-Vorrat 188 t C/ha.

MUCHIN et al. (2008) geben für die Baumartengruppe Kiefer in Brandenburg (Waldflächenanteil Kiefer > $70 \%$ ) eine Dendromasse von rund 82,7 Mio. $\mathrm{t}$ an, was in etwa $116 \mathrm{t} / \mathrm{ha}$ entspricht. Durch eine einfache Überschlagsrechnung ergeben sich in etwa $58 \mathrm{t} \mathrm{C/ha.} \mathrm{Umfangreiche} \mathrm{Untersuchungen} \mathrm{zur} \mathrm{Kiefer} \mathrm{wurden} \mathrm{in} \mathrm{Ost-}$ deutschland von HEINSDORF und KRAUB (1990) durchgeführt. Sie erstellten für vollbestockte Kiefernbestände unterschiedlicher Mittelhöhenbonität Schätztafeln mit der Angabe der Trockenmassen für verschiedene Baumkompartimente und der darin gespeicherten Nährstoffe. Danach hat z. B. ein 60-jähriger Kiefernbestand bei einer Mittelhöhe von 24 Metern einen C-Vorrat von 73,62 t C/ha in der oberirdischen Dendromasse.

\subsubsection{Biomassefunktionen}

\subsubsection{Getestete Biomassefunktionen}

In der Literatur sind viele verschiedene Biomassefunktionen beschrieben. Von diesen wurden aber nur die für niedersächsische Verhältnisse geeignet erscheinenden Funktionen untersucht. Diese sollen im Folgenden kurz vorgestellt werden.

Mit Hilfe von additiven Modellen ermittelte ZELL (2008) aus den Daten der BWI ${ }^{2}$ Funktionen zur Schätzung der oberirdischen Biomasse für acht Baumarten. Die in der Arbeit von PRETZSCH (2000) veröffentlichten Biomassefunktionen für Buche und Fichte bauen auf Daten von PELLINEN (1986) und ELLENBERG et al. (1996) auf, die aus dem Göttinger Wald bzw. Solling stammen.

In der Tschechischen Republik haben CIENCIALA et al. (2005, 2006, 2008) Funktionen zur Schätzung der Baumbiomasse für Buche, Kiefer und Eiche und 
deren oberirdischen Kompartimente entwickelt. BARTELINK (1997) stellte allometrische Funktionen für die oberirdische Biomasse von Buchen in den Niederlanden auf. Mit Daten aus Niederösterreich entwickelte HOCHBICHLER (2002) Funktionen für Eiche und Buche. WIRTH et al. (2004b) entwickelten eine Funktion für die ober- und unterirdische Biomasse der Fichte und ihre Kompartimente. Ihre Analysen beruhten auf 688 Bäumen aus 102 Studien von 19 Autoren. Auf der Grundlage einer Analyse von 13 mitteleuropäischen Studien leiteten WUTZLER et al. (2008) mit nichtlinearen gemischten Modellen Funktionen für die Biomassenschätzung bei Buche und ihre Kompartimente ab. ZIANIS et al. (2005) haben für viele Baumarten eine Übersicht der vorhandenen Biomasse- und Volumenfunktionen zusammengestellt. Unter anderem sind dort Funktionen für Fichte von FIEDLER (1986) und PoepPel (1989) aufgeführt. Durch eine Metaanalyse der Arbeit von ZIANIS et al. (2005) entwickelte MUUKKONEN (2007) allometrische Funktionen für die oberirdische Biomasse von Fichte, Kiefer, Buche, Eiche und Birke sowie deren Kompartimente.

Für die unterirdische Biomasse von Bäumen liegen sehr viel weniger Untersuchungen vor, weil ihre Erhebung sehr zeitaufwendig und kostenintensiv ist. Die Feinwurzelbiomasse ist dabei definitionsgemäß nicht in der unterirdischen Biomasse enthalten. Sie ist ein Bestandteil des Mineralbodens und wird daher nicht extra berechnet (s. Kap. 1.3). BOLTE et al. (2004) haben aus sechs Buchen-Fichtenmischbeständen im Solling Bäume beprobt, um Schätzfunktionen für die Wurzelbiomasse abzuleiten. Auch LE GOFF und OTTORINI (2001) führten entsprechende Untersuchungen für Buchen in Nordostfrankreich durch. Des Weiteren ist in der Literatur- und Datensammlung von JACOBSEN et al. (2003) der prozentuale Anteil verschiedener Baumkompartimente an der Gesamtbiomasse verschiedener Baumarten, unter anderem auch der der Grob- und Feinwurzelbiomasse, beschrieben. In Ermangelung geeigneter Methoden für Eiche und Kiefer sind für diese Baumarten die Buchen- bzw. Fichtenfunktionen von BOLTE et al. (2004) aufgeführt.

Eine tabellarische Übersicht der hier aufgezählten Funktionen mit ihren Koeffizienten ist im Anhang zu finden. Biomassefunktionen für die Hauptbaumarten in Nordwestdeutschland werden zurzeit im Rahmen des Projektes „Möglichkeiten und Grenzen der Vollbaumnutzung " an der NW-FVA erarbeitet, stehen aber noch nicht zur Verfügung.

\subsubsection{Auswabl geeigneter Biomassefunktionen}

Die zuvor genannten Biomassefunktionen wurden an einem vorhandenen Vergleichsdatensatz aus dem Datenpool der NW-FVA getestet. In ihm sind für die Baumarten Kiefer, Fichte, Buche und Traubeneiche der Brusthöhendurchmesser (BHD), die Höhe (H), die Biomasse und weitere Variablen enthalten. Von 1996 bis 2002 waren in verschiedenen Forstämtern Niedersachsens 72 Buchen, 71 Fichten, 31 Kiefern und 6 Traubeneichen geerntet und chemisch analysiert worden. Die 
Basisdaten dieser Erhebungen wurden als Eingangsdaten für die unterschiedlichen Funktionen verwendet, um die oberirdischen Biomassen zu schätzen und dann mit den gemessenen Biomassen zu vergleichen. Eine Übersicht über die Brusthöhendurchmesser und die Höhen der vier Baumarten des Referenzdatensatzes gibt Tabelle 2.

Tabelle 2: $\quad$ Übersicht des Referenzdatensatzes der NW-FVA mit Mittelwerten, Minimal- und Maximalwerten

\begin{tabular}{l|cc|cc|c}
\hline Baumart & $\begin{array}{c}\text { mittlerer } \\
\text { BHD } \\
{[\mathrm{cm}]}\end{array}$ & $\begin{array}{c}\text { BHD- } \\
\text { Spektrum } \\
{[\mathrm{cm}]}\end{array}$ & $\begin{array}{c}\text { mittlere } \\
\text { Höhe } \\
{[\mathrm{m}]}\end{array}$ & $\begin{array}{c}\text { Höhen- } \\
\text { spektrum } \\
{[\mathrm{m}]}\end{array}$ & Anzahl \\
\hline Buche & 28,62 & $8,2-55,6$ & 23,30 & $12,8-39,7$ & 72 \\
Eiche & 43,56 & $25,6-55,9$ & 20,80 & $17,2-23,4$ & 6 \\
Fichte & 37,96 & $13,2-73,8$ & 26,72 & $15,2-42,7$ & 71 \\
Kiefer & 18,09 & $4,5-45,8$ & 14,90 & $7,8-28,5$ & 31 \\
\hline
\end{tabular}

Vor der Analyse der Biomassefunktionen wurde zunächst der Referenzdatensatz nach Baumarten getrennt. Die einzelnen BHD- und Höhen-Werte decken ein weites Spektrum ab. Sie dienten anschließend als Eingangswerte für die verschiedenen, aus der Literatur zusammengestellten Biomassefunktionen, sodass die Biomasse jedes Baumes geschätzt werden konnte. Zur Beurteilung der Güte der Schätzungen wurden für die oberirdische Baumbiomasse der Bias und die Präzision als statistische Kenngrößen herangezogen, für die unterirdische Baumbiomasse der Mittelwert der geschätzten Biomasse, deren Standardabweichung und der daraus resultierende Variationskoeffizient. Letzterer ist ein relatives, dimensionsloses Streuungsmaß und erlaubt somit den Vergleich unterschiedlicher Datensätze (SACHS u. HEDDERRICH 2006, DORMANN u. KÜHN 2009).

Am Beispiel der Buche wird im Folgenden das Vorgehen zur Ermittlung der geeignetsten Funktion zur Schätzung der oberirdischen und unterirdischen lebenden Biomasse dargestellt. Die mittlere Baumbiomasse der Referenzdaten für Buche liegt bei $766 \mathrm{~kg} /$ Baum. Die geschätzten mittleren Biomassen für diese Baumart schwanken zwischen $722 \mathrm{~kg} / \mathrm{Baum}$ und $804 \mathrm{~kg} /$ Baum unter Verwendung der Funktionen von ZELL (2008) und BARTELINK (1997). In Tabelle 3 sind der Bias und die Präzision der verschiedenen Biomassefunktionen für Buche aufgeführt. Dabei wird der geringste Bias mit der Funktion von CiEnCIALA et al. (2005) und die höchste Präzision mit der Funktion von PRETZSCH (2000) erreicht. In Abbildung 10 sind die Ergebnisse der Biomasseschätzungen für Buche mit allen benutzten Funktionen den gemessenen Biomassen der 72 Buchen aus den Referenzdaten 
gegenübergestellt. Die Zacken in den Kurven treten bei den Funktionen auf, die die Baumhöhe als Eingangsparameter nutzen (s. Anhang).

Tabelle 3: $\quad$ Mittelwert, Bias und Präzision der verschiedenen Biomassefunktionen der oberirdischen Biomasse für Buche

\begin{tabular}{lccc}
\hline Autor & $\begin{array}{c}\text { Mittelwert } \\
{[\mathrm{kg} / \text { Baum }]}\end{array}$ & $\begin{array}{c}\text { Bias } \\
{[\mathrm{kg} / \text { Baum }]}\end{array}$ & $\begin{array}{c}\text { Präzision } \\
{[\mathrm{kg} / \text { Baum }]}\end{array}$ \\
\hline ZELL 2008 & 722,36 & $-44,4$ & 237,1 \\
WUTZLER et al. 2008 & 735,86 & $-30,9$ & 237,3 \\
PRETZSCH 2000 & 739,37 & $-27,4$ & 254,2 \\
CIENCIALA et al. 2005 & 764,27 & $-2,5$ & 233,6 \\
MUUKKONEN 2007 & 780,23 & $+13,4$ & 252,2 \\
BARTELINK 1997 & 804,37 & $+37,6$ & 251,6 \\
HOCHBICHLER 2002 & 783,98 & $+17,2$ & 233,6 \\
\hline
\end{tabular}

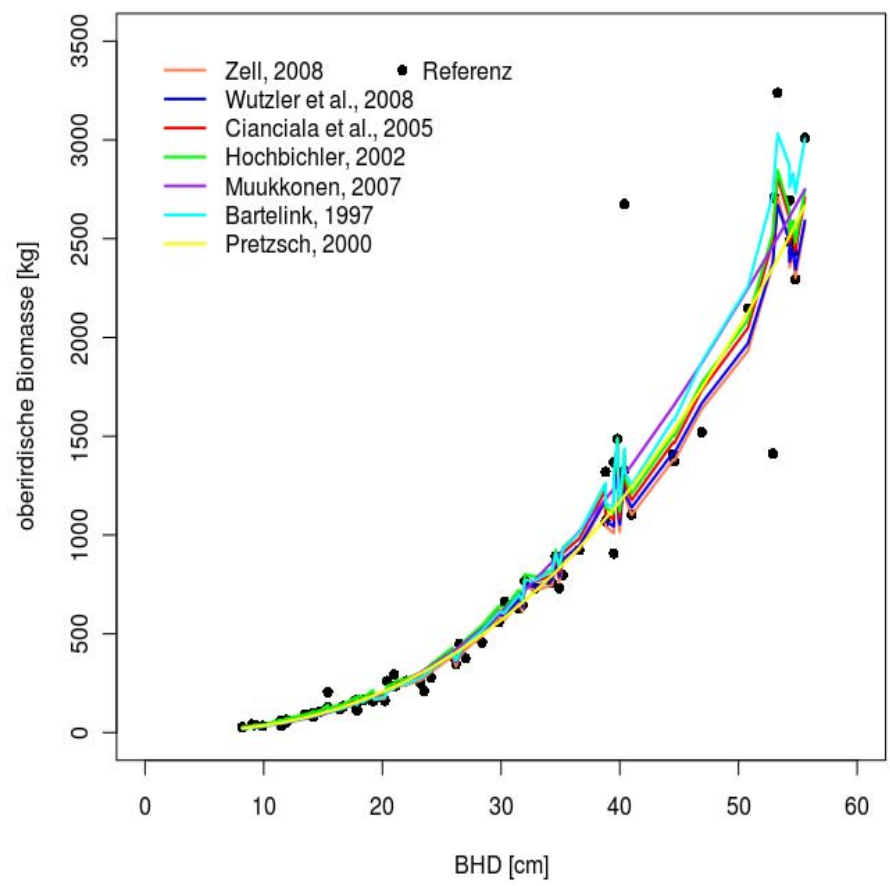

Abbildung 10: Ergebnisse der oberirdischen Biomasseschätzung aller untersucbten Biomassefunktionen für Buche mit den Referenzdaten 
Die Methoden zur Schätzung der unterirdischen lebenden Baumbiomasse stammen von BOLTE et al. (2004), WUTZLER et al. (2008) und LE GOFF u. OTTORINI (2001) (s. Anhang). Die mittlere geschätzte Grobwurzelbiomasse nach LE GOFF u. OTTORINI (2001) übertrifft diejenige nach BOLTE et al. (2004) um ein Vielfaches. Der geschätzte Mittelwert, hergeleitet mit der Funktion von WUTZLER et al. (2008), liegt zwischen denen der vorher genannten Methoden (s. Tab. 4 u. Abb. 11). Der Variationskoeffizient schwankt hier ebenfalls in einem geringen Bereich zwischen den unterschiedlichen Funktionen.

Tabelle 4: $\quad$ Ergebnisse der Schätzung der unterirdischen lebenden Baumbiomasse für Buche mit unterschiedlichen Funktionen

\begin{tabular}{lccc}
\hline Autor & $\begin{array}{c}\text { Mittelwert } \\
{[\mathrm{kg} / \text { Baum }]}\end{array}$ & $\begin{array}{c}\text { Standardabweichung } \\
{[\mathrm{kg} / \text { Baum }]}\end{array}$ & $\begin{array}{c}\text { Variationskoeffizient } \\
{[\%]}\end{array}$ \\
\hline BOLTE et al. 2004 & 48,09 & 47,45 & 98,66 \\
WUTZLER et al. 2008 & 120,02 & 123,2 & 102,65 \\
$\begin{array}{l}\text { LE GOFF u. } \\
\text { OTTORINI 2001 }\end{array}$ & 161,34 & 173,36 & 107,45 \\
\hline
\end{tabular}

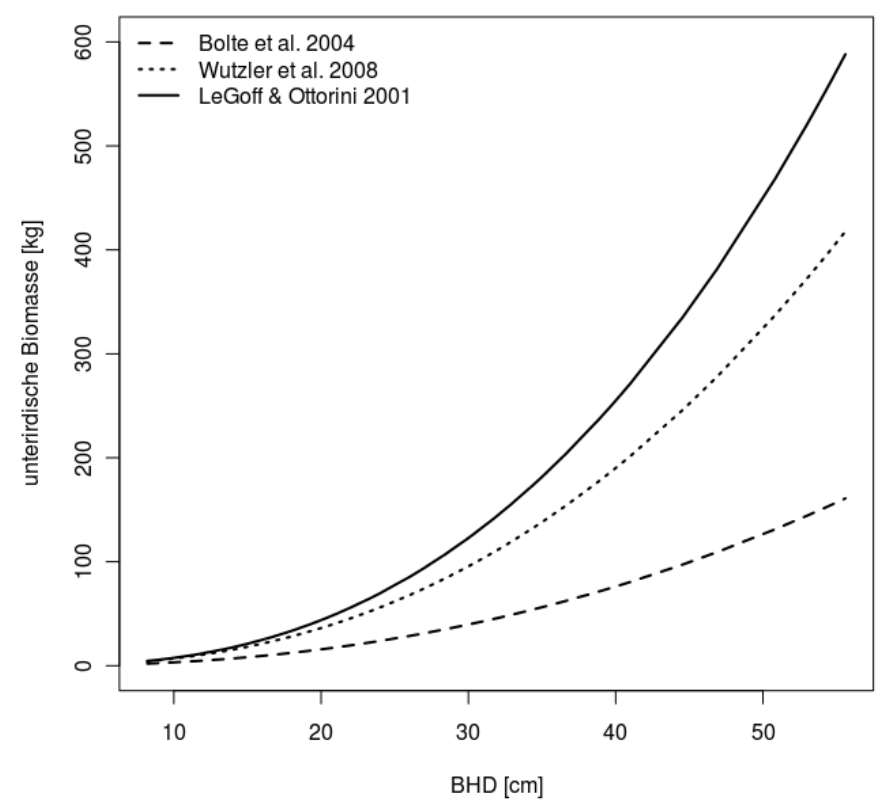

Abbildung 11: Vergleich der analysierten Biomassefunktionen zur Schätzung der unterirdischen lebenden Biomassen für Buche 
Die Überprüfung der vielen verschiedenen Biomassefunktionen aus dem europäischen Raum mit Hilfe des Referenzdatensatzes der NW-FVA zeigt, dass die Streuung der Ergebnisse zu einem großen Teil auf die jeweiligen Aufnahmegebiete mit ihren unterschiedlichen ökologischen Rahmenbedingungen (Einfluss auf die Ausformung der Bäume) zurückgeführt werden kann. Zudem sind die Daten in den verschiedenen Ländern bzw. Regionen methodisch unterschiedlich erhoben worden. Da Biomassefunktionen im Allgemeinen nur für bestimmte Gebiete und unter Umständen nur für bestimmte BHD-Bereiche entwickelt werden, ist ihre Anwendung in anderen Regionen bzw. für andere BHD-Bereiche immer mit Unsicherheiten verbunden. Auch sind unterschiedliche Biomassedefinitionen ein Grund für abweichende Ergebnisse, wie z. B. die eher subjektive Einteilung der oberirdischen holzigen Biomasse in Stamm und Äste im Vergleich zur objektiven Einordnung in Holz und Reisig mit festen Grenzwerten (Zopfdurchmesser 5 oder $7 \mathrm{~cm}$ ). Entsprechende Abweichungen finden sich auch bei der Definition der unterirdischen lebenden Baumbiomasse. So wird beispielsweise in der Arbeit von WUTZLER et al. (2008) nur von Grobwurzeln ohne erklärende Konkretisierungen gesprochen. Weitere Ursachen für die deutlich voneinander abweichenden Ergebnisse können auch Konkurrenzeffekte, schwankende klimatische Rahmenbedingungen oder unterschiedliche waldbauliche Behandlungen sein.

Nach den Ergebnissen der Vergleichsprüfungen werden in dieser Arbeit die baumartenspezifischen Funktionen von ZELL (2008) zur Berechnung der oberirdischen Biomasse herangezogen, die auf der Basis der BWI²-Daten entwickelt wurden. Sie weisen zwar einen hohen Bias und nur eine mittlere Präzision auf, bieten aber andererseits Vergleichsmöglichkeiten zu den Ergebnissen der BWI ${ }^{2}$ und sind allgemeingültig sowie durch eine geringe Streuung gekennzeichnet. Zur Berechnung der unterirdischen Wurzelbiomasse wird auf die Biomassefunktionen von BOLTE et al. (2004) für Buche und Fichte ohne Stubben zurückgegriffen, denen ein weiter BHD-Bereich zugrunde liegt und bei denen die Wurzelbiomasse genau als der unterirdische Teil des Wurzelstocks bis zu einem Wurzeldurchmesser von $2 \mathrm{~mm}$ definiert ist. In Ermangelung besserer Alternativen sollen diese Funktionen auch zur Bestimmung der unterirdischen Biomasse bei Eiche bzw. Kiefer genutzt werden.

\subsubsection{Berechnung der C-Vorräte der lebenden Baumbiomasse}

Für die Clusterstudie Forst und Holz Niedersachsen wurden Durchmesserwerte (BHD) und Höhenwerte auf Einzelbaumebene in 5-jährigen Intervallen simuliert. Diese Daten fanden Eingang in die baumartenspezifischen Funktionen von ZELL (2008) für die oberirdische lebende Baumbiomasse bzw. in die Funktionen von BOLTE et al. (2004) für die unterirdische lebende Baumbiomasse. Anhand der so berechneten Massen und der C-Konzentration (s. Kap. 3.1, erster Absatz) konnten die C-Vorräte der lebenden Baumbiomasse ermittelt werden. Mittels der Flächen- 
anteile der Baumarten konnten diese Werte auch pro Hektar zum Stichtag 31.12.2006 berechnet werden.

\subsection{Tote Baumbiomasse}

\subsubsection{Literaturstudie zur toten Baumbiomasse}

Das Totholz ist ein wichtiger Bestandteil des Ökosystems Wald, in dem sich große Mengen C akkumulieren können. PREGITZER und EUSKIRCHEN (2004) geben für Wälder der gemäßigten Zone einen C-Vorrat im Totholzpool von $42 \mathrm{t} \mathrm{C/ha}$ an. WIRTH et al. (2004a) ermittelten in ihrer Studie für extensiv genutzte Wälder in der borealen und gemäßigten Zone außerhalb Deutschlands einen Vorrat von 17,8 $\pm 16,9$ t C/ha. Für Nicht-Wirtschaftswälder in Mitteleuropa geben sie einen CVorrat von 5,9 $\pm 3,4 \mathrm{t} \mathrm{C} /$ ha und für Wirtschaftswälder von 2,1 $\pm 1,4 \mathrm{t} \mathrm{C} /$ ha an.

Bei der BWI ${ }^{2}$ (Stichjahr 2002) wurde im bundesweiten Durchschnitt ein Totholzvorrat ohne Stubben von $11,5 \mathrm{~m}^{3} / \mathrm{ha}(\mathrm{d} \geq 20 \mathrm{~cm}$ am stärkeren Ende) berechnet. Für Niedersachsen, Hamburg und Bremen liegt dieser Wert bei $8,2 \mathrm{~m}^{3} / \mathrm{ha}$ (d $\geq 20 \mathrm{~cm}$ ) (BMELV 2005). Nach den Ergebnissen der Inventurstudie 2008 hat sich der bundesweite Totholzvorrat auf insgesamt $15 \mathrm{~m}^{3} /$ ha erhöht. Bei Zugrundelegung einer geringeren Aufnahmegrenze $(\mathrm{d} \geq 10 \mathrm{~cm})$ beträgt der Totholzvorrat sogar $24 \mathrm{~m}^{3} /$ ha ohne Stubben (POLLEY et al. 2009). Zu noch höheren Werten bei gleicher abgesenkter Aufnahmegrenze kommen MEYER et al. (2009) auf der Basis der BZE II-Daten in den Bundesländern Hessen, Niedersachsen und SachsenAnhalt. Die von ihnen bestimmten Vorräte an Totholz belaufen sich auf $28 \mathrm{~m}^{3} / \mathrm{ha}$. Geht man von den zuvor genannten Volumina aus und unterstellt eine mittlere Dichte von $0,25 \mathrm{t} / \mathrm{m}^{3}$ für Totholz sowie einen C-Anteil von $50 \%$ an der Masse, so ergeben sich aus den Zahlen C-Vorräte von rund 1,44 t C/ha (BWI ${ }^{2}$, Dtschl., $\geq 20 \mathrm{~cm}$ ) und $1,02 \mathrm{t} \mathrm{C} / \mathrm{ha}\left(\mathrm{BWI}^{2}, \mathrm{Nds} ., \geq 20 \mathrm{~cm}\right)$ bzw. $1,84 \mathrm{t} \mathrm{C} / \mathrm{ha}$ (IS 2008, Dtschl., $\geq 20 \mathrm{~cm}$ ) bzw. $3 \mathrm{t} \mathrm{C} /$ ha (IS 2008, Dtschl., $\geq 10 \mathrm{~cm}$ ) und $3,5 \mathrm{t} \mathrm{C/ha}$ (MEYER et al. 2009, Nds., He., Sa.-Anh., $\geq 10 \mathrm{~cm}$ ). Diese Werte zeigen zum einen den starken Einfluss der Kluppschwellen auf die Totholzvorräte und die in ihnen gespeicherten C-Vorräte, zum anderen dokumentieren sie einen Totholzanstieg zwischen 2002 und 2008 aufgrund der höheren Waldrestholzanteile, die im Zuge der stärkeren Nutzungen im Wald verblieben sind. Zu betonen ist, dass in den Zahlen, die auf der BWI ${ }^{2}$ basieren, die Stubben nicht enthalten sind, welche aber viel Masse und auch einen wichtigen Lebensraum für viele Arten repräsentieren.

Die zeitliche Dynamik des Totholzpools wird bestimmt durch die Bildungsund Zersetzungsraten des Totholzes. Eine Zunahme erfährt der Totholzvorrat durch die natürliche Mortalität und den Astabfall, abiotisch bzw. biotisch bedingte Ausfälle sowie durch menschliche Eingriffe. Die Zersetzung des Holzes ist der Hauptaustrag. Er wird vor allem von Destruenten vorangetrieben, deren Atmung zu einer direkten Freisetzung von C führt (MACKENSEN et al. 2003). Die Zerset- 
zungsdauer von Totholz ist bei der Quantifizierung der C-Speicherung in Waldökosystemen ein wichtiger Aspekt. Beispielsweise veröffentlichten ROCK et al. (2008) kontinuierliche Zersetzungskonstanten für die Hauptbaumarten in Europa. ZELL et al. (2009) leiteten ein gemischtes nichtlineares Abbaumodell auf der Basis einer Metaanalyse her, in das unter anderem der Ausgangsdurchmesser des Totholzes, die mittlere Temperatur im Juli und der Jahresniederschlag als erklärende Variablen eingehen. MEYER et al. (2009) haben in ihrer Arbeit unter anderem Totholzzersetzungsfunktionen $(\mathrm{d} \geq 20 \mathrm{~cm})$ für die Hauptbaumarten in Niedersachsen ableiten können.

\subsubsection{Berechnung der C-Vorräte der toten Baumbiomasse}

In den Simulationsläufen der Clusterstudie Forst und Holz Niedersachsen wurden für die verschiedenen Szenarien auch die Totholzvolumina hergeleitet. Totholz ist darin definiert als das oberirdische tote Derbholz mit einem Mindestdurchmesser von $7 \mathrm{~cm}$. Den jeweiligen Totholzvolumina wurden die Erntereste aus den Vorund Endnutzungen hinzuaddiert. Diese wurden mit Hilfe baumartenspezifischer Abzugsfaktoren ${ }^{3}$ quantifiziert. Die im Rahmen der BWI 2002 mit einem Durchmesser $\geq 20 \mathrm{~cm}$ ermittelten Totholzvolumina in Höhe von $8,1 \mathrm{~m}^{3} / \mathrm{ha}$ (ML 2004) wurden mit Hilfe einer Funktion von MEYER et al. (2009), die den Zusammenhang zwischen dem ermittelten Totholzvolumen und dem Mindestdurchmesser bei den Totholzaufnahmen beschreibt, für die Aufnahmegrenze $\geq 7 \mathrm{~cm}$ auf $14,4 \mathrm{~m}^{3} / \mathrm{ha}$ bzw. insgesamt 15,7 Mio. $\mathrm{m}^{3}$ umgerechnet. Zu diesem Wert wurden die Erntereste von 2002 bis Ende 2006 in Höhe von 3,9 Mio. $\mathrm{m}^{3}$ hinzuaddiert. Auf dieser Grundlage konnte für alle Bestände ein Startwert generiert werden.

Der Totholzabbau durch Zersetzung wurde für die Hauptbaumarten mit den Funktionen von MEYER et al. (2009) abgebildet. Das Gesamtvolumen der toten Baumbiomasse wurde anschließend mit einer mittleren Raumdichte für Laub$\left(0,3 \mathrm{t} / \mathrm{m}^{3}\right)$ bzw. Nadelholz $\left(0,21 \mathrm{t} / \mathrm{m}^{3}\right)$ in Masse umgerechnet, sodass der C-Vorrat unter Zugrundelegung einer C-Konzentration von $50 \%$ berechnet werden konnte. Die mittlere Raumdichte wurde ermittelt, indem die Raumdichten in unterschiedlichen Zersetzungsstadien mit der Verweildauer in der jeweiligen Zersetzungsphase gewichtet und anschließend gemittelt wurden. Dazu dienten die Angaben von FRAVER et al. (2002) und RANIUS (2003) für Nadelholz sowie von MÜLLERUsING und BARTSCH (2009) für Laubholz.

3 Vornutzung: Buche $=0,15$; Eiche $=0,25$; Fichte $=0,2$; Kiefer $=0,25$

Endnutzung: Buche $=0,15$; Eiche $=0,25 ;$ Fichte $=0,15 ;$ Kiefer $=0,2($ KRAMER u. AKÇA 2008)

Beiträge aus der NW-FVA, Band 6, 2011 


\subsection{Bodenvegetation}

Der bestimmende Faktor für die C-Speicherung in der Bodenvegetation ist die auf den Waldboden treffende Strahlungsmenge, die sehr unterschiedlich sein kann (BÖSWALD u. WIERLING 1997). So erhält z. B. der Waldboden eines hallenartigen Buchenaltbestandes völlig andere Strahlungsmengen als der eines geschlossenen jungen Buchenbestandes mit tief ineinandergreifenden Kronen. Zur Ermittlung der C-Vorräte in der Bodenvegetation liegen keine flächendeckenden Daten für Niedersachsen vor bzw. eine Regionalisierung dieser Daten aus Stichprobeninventuren ist schwierig. In der Clusterstudie Forst und Holz Niedersachsen war die Bodenvegetation weder integriert noch wurde sie fortgeschrieben. Aus diesem Grund wurde für die C-Studie in Niedersachsen behelfsweise auf die Ergebnisse einer Studie für Thüringen zurückgegriffen (WIRTH et al. 2004a). Die Bodenvegetation in Buchenbeständen hat danach einen C-Vorrat von $0,3 \mathrm{t} \mathrm{C/ha}$, in Eichenbeständen 1,69 t C/ha, in Fichtenbeständen 0,24 t C/ha und in Kiefernbeständen 1,46 t C/ha (WIRTH et al. 2004a). Da sich die Bodenvegetation größtenteils aus Pflanzen mit einer geringen Lebensspanne zusammensetzt, wurde der C-Vorrat in diesem Pool als konstant angesehen und nicht weiter betrachtet.

\subsection{Boden}

\subsubsection{Literaturstudie zum Boden}

Im Ökosystem Wald ist der Boden meist ein größerer Kohlenstoffspeicher als die Vegetation. Auf dem ersten Meter im Boden beträgt der C-Vorrat in den Wäldern der borealen Zone schätzungsweise $338 \mathrm{Pg}^{4}$, in der gemäßigten Zone 153 bis $195 \mathrm{Pg}$ und in der tropischen Zone 214 bis 435 Pg (SAUGIER et al. 2001). Das durchschnittliche Verhältnis des C-Vorrates im Boden zur darauf befindlichen Vegetation beträgt in der borealen Zone etwa 5:1, in der gemäßigten 2:1 und in der tropischen 1:1 (JARVIS et al. 2005).

In Thüringen wurden die C-Vorräte in der organischen Auflage der Wälder auf 27,7 t C/ha geschätzt. Dieser C-Vorrat im Humus war in den Nadelwäldern im Mittel dreimal höher als in den Laubwäldern. Im Mineralboden $(0-60 \mathrm{~cm})$ waren 69,9 t C/ha gespeichert, wobei diese Werte unter Laubwald leicht höher waren als unter Nadelwald (WIRTH et al. 2004a). In der Arbeit von PISTORIUS (2007) wurden für Baden-Württemberg im Mineralboden sehr viel höhere C-Vorräte durch Simulationsrechnungen ermittelt $(135,4 \mathrm{tC} / \mathrm{ha})$, die aber auf keine bestimmte Tiefenstufe bezogen und daher schwer einzuordnen sind. In der Auflage waren die C-Vorräte (27,6 t C/ha) ähnlich hoch wie in Thüringen.

$41 \mathrm{Pg}=1$ Peta Gramm $=1 * 10^{15} \mathrm{~g}=1 * 10^{9} \mathrm{t}=1 \mathrm{Giga}$ Tonne $=1 \mathrm{Gt}$ 


\subsubsection{Berechnung der C-Vorräte des Bodens}

Zur Berechnung des C-Vorrates in den Böden der niedersächsischen Wälder dienten die Daten der BZE II. Es standen sowohl Aufnahmen für den Mineralboden und für die Humusauflage sowie für Moorstandorte zur Verfügung.

Insgesamt konnten Daten von 149 BZE II-Profilen genutzt werden, um den mittleren C-Vorrat in der Humusauflage zu ermitteln. Der C-Vorrat der niedersächsischen Mineralböden wurde anhand von 27 BZE II-Profilen vorläufig berechnet. Bei diesen Profilen wurde nicht die Satellitenstichprobe, sondern die Probe am Bodenprofil als Datengrundlage aus den oben genannten Gründen (s. Kap. 2.3.2) herangezogen. Für die Moorböden mit ihren sehr hohen C-Vorräten erfolgten gesonderte Auswertungen. Zu den BZE-Punkten lagen weiterhin auch Bestandesinformationen aus Probekreisaufnahmen vor, die zur Verschneidung mit den Bodeninformationen genutzt wurden.

\subsection{Holzprodukte}

\subsubsection{Literaturstudie Holzprodukte}

Der Produktpool Holz stellt neben dem Wald einen weiteren Speicher für Kohlenstoff dar, der in dieser Studie betrachtet wird. Holzprodukte leisten einen nennenswerten Beitrag zur Reduzierung der $\mathrm{CO}_{2}$-Konzentrationen in der Atmosphäre, indem durch die stoffliche Holznutzung die natürliche Freisetzung dieses Treibhausgases durch Zersetzung verzögert wird. Außerdem substituieren Holzprodukte sowohl andere Bau- und Werkstoffe, die in der Herstellung energieintensiver sind und größere $\mathrm{CO}_{2}$-Emissionen verursachen, als auch als Energieholz fossile Brennstoffe (KÖHL et al. 2009, RÜTER 2010). Durch den steigenden stofflichen Holzverbrauch konnten in den vergangenen Jahren bundesweit durchschnittlich 10 Mio. t/Jahr $\mathrm{CO}_{2}$-Emissionen und durch die wachsende energetische Holznutzung weitere $30 \mathrm{Mio} . \mathrm{t} / \mathrm{Jahr} \mathrm{CO}_{2}$-Emissionen zusätzlich vermieden werden (RÜTER 2010).

In einer Schweizer Studie wurde für Holzprodukte im Zivilisationskreislauf ein C-Vorrat von 101 Mio. t $\mathrm{CO}_{2}$ im Jahr 2000 geschätzt, was ca. 27,5 Mio. t C entspricht (TAVERNA et al. 2007). BÖSWALD und WIERLING (1997) berechneten für den niedersächsischen Holzproduktpool eine Speichermenge von 23,8 Mio. t C im Jahr 1993. PISTORIUS (2007) kam für Baden-Württemberg auf der Basis statistischer Angaben zur Gebäudezahl und den Nutzungsmengen zwischen BWI ${ }^{1}$ und $\mathrm{BWI}^{2}$ zu einer Speicherleistung von 49,85 Mio. t C für den Produktpool Holz. Weiterhin konnte er eine jährliche zusätzliche Speicherung von etwa 0,85 Mio. t C ermitteln. Die mittlere Kohlenstoffbindung im verkauften Holz des Thüringer Landeswaldes betrug in den Jahren 2001 und 2002 rund 0,21 Mio. t C (MUND et al. 2006). 
Wichtige Informationen zur Einschätzung der C-Bindung in Holzprodukten sind die Absatzwege des sägefähigen und nicht sägefähigen Rohholzes. Für die Verwendung bestimmter Holzsortimente liegen aber bisher weder für Niedersachsen noch für Deutschland belastbare baumartenspezifische Daten vor. Lediglich für Thüringen gibt es Näherungswerte aus einer Untersuchung von PROFFT et al. (2009). Besser ist die Informationslage hinsichtlich der mittleren Lebensdauer einzelner Produktklassen (WIRTH et al. 2004a).

Bei einer vollständigen Nutzung des Holzes als langlebiges Produkt (Materialsubstitution) mit darauf folgender energetischer Nutzung (Energiesubstitution) ergibt sich ein maximales Substitutionspotenzial von 1,238 t C pro t Holz (RoCK 2008). Dieser Wert stellt eine Obergrenze dar, da nicht alles Holz zu langlebigen Produkten verarbeitet wird, mit diesen nicht immer fossile Brennstoffe ersetzt werden und ihre Verbrennung nicht zwangsläufig von Nutzen sein muss (ROCK 2008). In jedem Fall ist der Trend zu beachten, dass Holz als Energieträger immer wichtiger wird. Nach einer Studie von MANTAU et al. (2010) wird der Anteil der energetischen Nutzung bei steigendem Holzverbrauch von heute $43 \%$ auf $54 \%$ im Jahre 2030 steigen. Im Jahre 2002 betrug dieser Anteil lediglich 26 \% (MANTAU 2004). Auch in Niedersachsen ist Holz als Energieträger immer wichtiger geworden. Allein in Feuerungsanlagen unter 1 Megawatt Leistung wurden 2009 durch Energieholznutzung 4,78 Mio. Megawattstunden (MWh) an fossilen Energieträgern substituiert (3N 2010).

\subsubsection{Kaskadennutzung}

In dieser C-Studie wird der Produktpool als statisch angesehen, d. h. es findet kein Übergang von einer Holzproduktklasse in eine andere statt. Jedoch ist es durch Kaskadennutzung generell möglich, die Lebensdauer von Holzprodukten zu verlängern bzw. durch eine energetische Nutzung von Altholz die Substitutionseffekte durch Holzprodukte zu vergrößern. Kaskadennutzung bedeutet im klassischen Sinn die stoffliche Nutzung eines Rohstoffs über mehrere Stufen, wobei im ersten Produkt die stofflichen Eigenschaften auf dem höchsten Niveau genutzt werden. Am Ende jeder Produktlebensdauer erfolgt eine Aufbereitung des Rohstoffs, um ihn dann anschließend zu einem anderen Produkt zu verarbeiten. Dieses neue Produkt stellt in der Regel geringere Anforderungen an die stofflichen Eigenschaften des Rohstoffs als das vorherige. Somit wird der Rohstoff weiter verwertet, anstatt dass er neu hergestellt oder beschafft werden muss. Dabei ist die Häufigkeit der Wiederverwertung abhängig vom eingesetzten Rohstoff sowie technischen, sozialen, ökologischen und ökonomischen Rahmenbedingungen. Durch eine Kaskadennutzung wird demnach die stoffliche Ressource durch wiederholten Einsatz optimal genutzt und zeitgleich werden die geringer werdenden Qualitäten durch die verminderten Anforderungen an die Folgeprodukte berücksichtigt (FRÜHWALD et al. 2010). In der Realität ist die Kaskadennutzung nicht rein linear, sondern auf 
den verschiedenen Nutzungsstufen werden Stoffe zur energetischen Verwendung abgezweigt.

FRÜHWALD et al. (2010) haben dies in ihrer Arbeit beispielhaft für Holz dargestellt. Für das erste Produkt, etwa konstruktives Bauholz, wird Schnittholz benötigt, welches dann beim kontrollierten Rückbau von Häusern als Altholz aussortiert werden kann. Dieses Altholz kann anschließend für die Spanplattenproduktion genutzt werden. In Deutschland fallen jährlich 8 Mio. Tonnen Altholz an, von denen $80 \%$ energetisch und $20 \%$ stofflich weiterverwendet werden. Die energetische Verwertung von Holz stellt die letzte Nutzungsstufe dar. Durch die Kaskadennutzung wird die Gesamtnutzungsdauer des Holzes und damit die CSpeicherung in Holzprodukten verlängert, ohne dass die Holzentnahme aus den Wäldern erhöht werden muss (FRÜHWALD et al. 2010).

\subsubsection{Berechnungen zum Produktpool}

\subsubsection{Holzproduktklassen}

Die Holzprodukte wurden in vier Kategorien eingeteilt, die sich aus verschieden Hauptprodukten zusammensetzen (s. Tab. 5). Bei diesen vier Holzproduktklassen handelt es sich um „Produkte mit langer Lebensdauer“ (PIL), „Produkte mit mittlerer Lebensdauer“ (PmL), „Produkte mit kurzer Lebensdauer“ (PkL) und „Energieholz“ (E). Diesen Produktklassen wurden Zersetzungskonstanten (k) auf Grundlage der Angaben von WIRTH et al. (2004a) und PISTORIUS (2007) zugeordnet. Damit war es möglich, für die einzelnen Klassen Halbwertszeiten ( $\left.t_{50}\right)$, mittlere Lebensdauern $\left(\mathrm{t}_{63}\right)$ und Lebensdauern $\left(\mathrm{t}_{95}\right)$ zu berechnen (s. Gleichung 1). Die Lebensdauern ( $\mathrm{t}$ ) entsprechen einem exponentiellen Abbau nach der Zeit, in der $50 \%, 63 \%$ und $95 \%$ (i) des organischen Kohlenstoffs abgebaut sind (MUND et al. 2006).

$$
t_{i}=-\left(\ln \left(1-\frac{i}{100}\right)\right) / k
$$

Diese Unterscheidung ist nötig, da bei einem exponentiellen Abbau mit einer konstanten Zersetzungsrate angenommen wird, dass die gesamte Lebensdauer ( $\left.\mathrm{t}_{95}\right)$, d. h. die Zeitspanne, nach deren Ablauf noch $5 \%$ der Ausgangsmenge verblieben sind, mehr als doppelt so lang ist wie die mittlere Lebensdauer ( $\left.\mathrm{t}_{63}\right)$ (MUND et al. 2006). Die Halbwertszeit ( $\left.t_{50}\right)$ entspricht dabei dem Zeitpunkt, an dem $50 \%$ der Masse abgebaut sind, was aber nicht mit der mittleren Lebensdauer $\left(t_{63}\right)$ gleichzusetzen ist. 
Tabelle 5: Aufstellung der Produktklassen mit den entsprechenden Zersetzungskonstanten (k), Lebensdauern $\left(t_{50}=\right.$ Halbwertszeit, $t_{63}=$ mittlere Lebensdauer, $t_{95}=$ Lebensdauer $)$ und Hauptprodukten. (WIRTH et al. 2004a, PISTORIUS 2007; Lebensdauern berechnet nach Gleichung 1)

\begin{tabular}{|c|c|c|c|c|c|c|}
\hline \multicolumn{2}{|c|}{ Produktklasse } & $\begin{array}{c}\text { Zerset- } \\
\text { zungskon- } \\
\text { stante (k) } \\
\text { [ahre-1] }\end{array}$ & $\begin{array}{c}\text { Halb- } \\
\text { wertszeit } \\
\left(\mathrm{t}_{50}\right) \\
\text { [Jahre] }\end{array}$ & $\begin{array}{c}\text { Mittlere } \\
\text { Lebens- } \\
\left.\text { dauer (t } \mathrm{t}_{63}\right) \\
\text { [Jahre] }\end{array}$ & $\begin{array}{c}\text { Lebens- } \\
\text { dauer } \\
\left(\mathrm{t}_{95}\right) \\
\text { [Jahre] }\end{array}$ & $\begin{array}{l}\text { Haupt- } \\
\text { produkte }\end{array}$ \\
\hline \multirow{3}{*}{$\begin{array}{l}\text { Produkte } \\
\text { mit }\end{array}$} & $\begin{array}{l}\text { langer } \\
\text { Lebens } \\
\text { dauer }\end{array}$ & 0,02 & 35 & 50 & 150 & Bauholz \\
\hline & $\begin{array}{l}\text { mitt- } \\
\text { lerer } \\
\text { Lebens } \\
\text { dauer }\end{array}$ & 0,039 & 18 & 25 & 77 & $\begin{array}{l}\text { Holzwerk } \\
\text { stoffe wie } \\
\text { Span- und } \\
\text { Tischler- } \\
\text { platten, } \\
\text { Furniere } \\
\text { und Voll- } \\
\text { holz- } \\
\text { möbel }\end{array}$ \\
\hline & $\begin{array}{l}\text { kurzer } \\
\text { Lebens } \\
\text { dauer }\end{array}$ & 0,32 & 2 & 3 & 9 & $\begin{array}{l}\text { Papier, } \\
\text { Pappe, } \\
\text { Karto- } \\
\text { nagen }\end{array}$ \\
\hline \multicolumn{2}{|c|}{ Energieholz } & 0,7 & 1 & 1 & 4 & $\begin{array}{l}\text { Brenn- } \\
\text { holz, } \\
\text { Pellets }\end{array}$ \\
\hline
\end{tabular}

\subsubsection{Holzverwendungsschliussel}

Ein Holzverwendungsschlüssel dient der Zuordnung verschiedener Sortimente zu unterschiedlichen Produkten und Produktklassen. Die Erstellung eines Holzverwendungsschlüssels ist zeitaufwendig und stellt hohe Ansprüche an die Datengrundlagen. Probleme ergeben sich dabei dadurch, dass sich die Wege der verschiedenen ausgehaltenen Sortimente häufig nicht vom Wald zum Verwerter nachvollziehen lassen (Verkäufer $\rightarrow$ Verwerter oder Holzhandel $\rightarrow$ Verarbeitung oder Weiterverkauf). Dies ließe sich ggf. mit einer umfassenden Befragung der Unternehmen der holzbe- und -verarbeitenden Industrie eines Landes bewerkstelligen (vgl. PROFFT et al. 2009), was aber im Rahmen dieser Studie nicht möglich war. 
Mit Hilfe des Landesbetriebs für Statistik und Kommunikationstechnologie Niedersachsen (LSKN) wurde daher versucht, einen Verwendungsschlüssel auf Grundlage der Meldenummern der Zentralen Markt- und Preisberichtstelle GmbH i. L. (ZMP) für die Jahre 2004 bis $2008 \mathrm{zu}$ erstellen. Dies scheiterte jedoch an der meist fehlenden Unterscheidung zwischen Laub- und Nadelholz im Datensatz.

Stark vereinfacht wurde daher folgender Ansatz gewählt: Den Produktklassen wurde ausgehend von den Hauptprodukten das eingeschlagene Stamm-, Industrieund Energieholz sowie das nicht verwertbare Holz grob zugeordnet. Stammholz wird zumeist an Säger verkauft, welche daraus Produkte für den Hausbau o. ä. anfertigen. Folglich wird dieses Sortiment der Produktklasse mit langer Lebensdauer (PIL) zugeordnet. Der Ausbeutungsgrad bei der Schnittholzproduktion beträgt bundesweit für Nadelholz ca. $61 \%$ und für Laubholz ca. $64 \%$ (MANTAU 2008). Die als Koppelprodukt entstehenden Sägenebenprodukte werden, unabhängig ob es sich um Laub- oder Nadelholz handelt, zu $44 \%$ der Klasse Produkte mit mittlerer Lebensdauer, zu $18 \%$ der Klasse Produkte mit kurzer Lebensdauer und zu 38 \% der Klasse Energieholz zugeordnet. Das eingeschlagene Industrieholz wird $\mathrm{zu}$ unterschiedlichen Anteilen den Produkten mit mittlerer (PmL) und kurzer Lebensdauer (PkL) zugewiesen. Um den Anteil von Laub- und Nadelindustrieholz an der Kategorie Produkte mit kurzer Lebensdauer, hauptsächlich Papier und Pappe, zu bestimmen, wurde auf bundesweite Zahlen des Verbandes Deutscher Papierfabriken e.V. (VDP) zurückgegriffen, da Daten auf Landesebene nicht vorlagen (VDP 2007). Das Nadelindustrieholz wurde demnach zu $64 \%$ und das Laubindustrieholz zu $27 \%$ dieser Produktklasse zugeordnet. Ausgehaltenes Energieholz und nicht verwertbares Holz wurde der Produktklasse Energieholz zugeordnet. Die Datengrundlage für diese Sortimente stammt aus dem Holzmarktbericht 2007 (BMELV 2008) für das Jahr 2006. Dieses Jahr wurde gewählt, da es ein von Stürmen und Wirtschaftsschwankungen weitgehend unbeeinflusstes Jahr war. Der stark vereinfachte Holzverwendungsschlüssel ist in Tabelle 6 dargestellt.

Tabelle 6: $\quad$ Stark vereinfachter Holqverwendungsschlüssel für das Land Niedersachsen. Dargestellt sind die jeweiligen Anteile von Laub-oder Nadelholz an den verschiedenen Produktkelassen.

\begin{tabular}{lcccc}
\hline Art & $\begin{array}{c}\text { Produkte mit } \\
\text { langer } \\
\text { Lebensdauer }\end{array}$ & $\begin{array}{c}\text { mittlerer } \\
\text { Lebensdauer }\end{array}$ & $\begin{array}{c}\text { kurzer } \\
\text { Lebensdauer }\end{array}$ & Energieholz \\
\hline Laubholz & $24,7 \%$ & $24,5 \%$ & $9,4 \%$ & $41,4 \%$ \\
Nadelholz & $34,7 \%$ & $23,4 \%$ & $27,9 \%$ & $14,0 \%$ \\
\hline
\end{tabular}


Mit dem Holzverwendungsschlüssel wurden die simulierten Nutzungsmengen der Clusterstudie Forst und Holz Niedersachsen den Produktklassen zugeordnet. Die jeweiligen Volumina wurden anschließend mit baumartenspezifischen Raumdichten $^{5}$ in Massen und anschließend mit der C-Konzentration von $50 \%$ in CVorräte umgerechnet.

Die Berechnung der Substitutionseffekte beruht auf Untersuchungen, wonach bei der Verbrennung von Nadelholz mit einem Wassergehalt von $20 \%$ im Vergleich zur entsprechenden Menge Heizöl 1,401 t CO 2 pro verwendeter Tonne Holz eingespart werden. Bei Laubholz liegt dieser Wert bei 1,345 $\mathrm{t} \mathrm{CO}_{2} / \mathrm{t}$ Holz (PFLÜGER-Grone 2007). Die C-Vorräte der Produktklasse Energieholz wurden mit dem Atommassenverhältnis von 3,67 in $\mathrm{CO}_{2}$-Werte (s. Kap. 3.1, erster Absatz) umgewandelt und mit den eben genannten Zahlen multipliziert, um den energetischen Substitutionseffekt darstellen zu können.

5 Raumdichten: Eiche: $561,1 \mathrm{~kg} / \mathrm{m}^{3}$, Buche: $554,3 \mathrm{~kg} / \mathrm{m}^{3}$, Kiefer: $430,7 \mathrm{~kg} / \mathrm{m}^{3}$, Fichte: $377,1 \mathrm{~kg} / \mathrm{m}^{3}$ (KNIGGE u. SCHULZ 1966) 



\section{Ergebnisse}

Einleitend werden die Kohlenstoffvorräte in Niedersachsens Wäldern zu den Stichtagen der BWI 1987 und der BWI² 2002 sowie Ende 2006 vorgestellt und die Veränderungen beschrieben. Danach werden die Auswirkungen der drei waldbaulichen Szenarien auf die verschiedenen C-Speicher dargestellt. Zum 01.01.2007 beginnen die Simulationsrechnungen der Clusterstudie Forst und Holz Niedersachsen. Bis zu diesem Zeitpunkt wurden die Vorräte der BWI ${ }^{2}$ unter Berücksichtigung der tatsächlichen Zuwächse und Einschläge im Zeitraum 2002 bis Ende 2006 fortgeschrieben sowie um die Kalamitätsnutzungen nach dem Orkan Kyrill vom 18./19.01.2007 korrigiert.

\subsection{Veränderungen zwischen 1987 und 2002}

Der Gesamtderbholzvorrat 1987 (BWI') betrug in Niedersachsen für alle Baumarten rund 199 Mio. $\mathrm{m}^{3}$ bzw. $197 \mathrm{~m}^{3} /$ ha. Darin waren geschätzte 48 Mio. t C bzw. 47 t C/ha gespeichert. Bis zum Zeitpunkt der BWI2 stieg der Derbholzvorrat um mehr als 100 Mio. $\mathrm{m}^{3}$ auf $305 \mathrm{Mio}^{3}$ an, was einem durchschnittlichen Vorrat von $279 \mathrm{~m}^{3} /$ ha entspricht. Gleichzeitig erhöhte sich der C-Vorrat auf ca. 76 Mio. t C bzw. 70 t C/ha (s. Abb. 12).

Der Derbholzvorrat der Baumartengruppe Buche betrug zum Stichtag der BWI ${ }^{1}$ rund 45,8 Mio. $\mathrm{m}^{3}$ bzw. $325 \mathrm{~m}^{3}$ /ha. Dies entspricht etwa 12,7 Mio. t C bzw. 90 t C/ha. Geringer waren die Derbholzvorräte bei der Baumartengruppe Eiche (20,3 Mio. $\mathrm{m}^{3} ; 240 \mathrm{~m}^{3} / \mathrm{ha}$ ) mit ebenfalls geringeren C-Vorräten (5,7 Mio. t C; $67 \mathrm{tC} / \mathrm{ha})$. Für die Nadelbaumarten mit ihrem damaligen Überhang in der II. Altersklasse wurden Derbholzvorräte von insgesamt 47,7 Mio. $\mathrm{m}^{3}$ bzw. $228 \mathrm{~m}^{3}$ /ha bei Fichte und 59,2 Mio. $\mathrm{m}^{3}$ bzw. $164 \mathrm{~m}^{3} /$ ha bei Kiefer ermittelt. Die darin gespeicherten C-Vorräte beliefen sich auf ca. 9,0 Mio. t C bzw. 43 t C/ha bei Fichte und ca. 12,7 Mio. t C bzw. 35 t C/ha bei Kiefer. Die Quantifizierung erfolgte vereinfacht auf der Grundlage der Derbholzvorräte, da hier keine Biomassefunktionen angewendet werden konnten. Für die Umrechnung des Volumens in Masse wurden die baumartenspezifischen Raumdichten verwandt.

Die C-Vorräte der Hauptbaumarten zum Stichtag der BWI2 sind ein Spiegelbild ihrer Flächenanteile, ihrer standörtlichen Zuordnung und ihres Altersklassenaufbaus (s. Abb. 4).

Die Buchenbestände mit ihrem Altholzüberhang hatten 2002 einen Derbholzvorrat von 56,5 Mio. $\mathrm{m}^{3}$ mit einer Masse von 39 Mio. t. Der C-Vorrat in der lebenden Baumbiomasse belief sich auf 19,5 Mio. t C (s. Tab. 7). Die durchschnittlichen Hektarwerte betrugen $374,9 \mathrm{~m}^{3} /$ ha für den Derbholzvorrat, $242 \mathrm{t} /$ ha für die 
lebende oberirdische Baumbiomasse, 16,6 t/ha für die lebende unterirdische Baumbiomasse und 129,3 t C/ha für den darin gespeicherten C-Vorrat.

Tabelle 7: $\quad$ Derbholzvorräte, lebende Baumbiomassen und C-Vorräte für die Stichjabre 1987, 2002 und Ende 2006 getrennt nach Baumarten (1987: nicht funktional hergeleitet, 2006: Werte pro ha sind Näherungswerte)

\begin{tabular}{|c|c|c|c|c|c|c|c|}
\hline \multirow[t]{2}{*}{ Baumart } & \multirow[t]{2}{*}{ Jahr } & \multicolumn{2}{|c|}{ Derbholzvorrat } & \multicolumn{2}{|c|}{$\begin{array}{c}\text { Lebende } \\
\text { Baumbiomasse }\end{array}$} & \multicolumn{2}{|c|}{ C-Vorrat } \\
\hline & & $\begin{array}{c}{\left[\text { Mio. } \mathrm{m}^{3}\right]} \\
\left(\mathrm{m}^{3} / \mathrm{ha}\right)\end{array}$ & $\%$ & $\begin{array}{l}\text { [Mio. t] } \\
(\mathrm{t} / \mathrm{ha})\end{array}$ & $\%$ & $\begin{array}{c}\text { [Mio. t C] } \\
(\mathrm{t} \mathrm{C} / \mathrm{ha})\end{array}$ & $\%$ \\
\hline \multirow{3}{*}{ Buche } & 1987 & $\begin{array}{c}45,8 \\
(325,0)\end{array}$ & 100 & $\begin{array}{c}25,4 \\
(180,0)\end{array}$ & 100 & $\begin{array}{c}12,7 \\
(90,0)\end{array}$ & 100 \\
\hline & 2002 & $\begin{array}{c}56,5 \\
(374,9)\end{array}$ & 123 & $\begin{array}{c}39,0 \\
(258,6)\end{array}$ & 154 & $\begin{array}{c}19,5 \\
(129,3)\end{array}$ & 153 \\
\hline & 2006 & $\begin{array}{c}58,6 \\
(389,2)\end{array}$ & 128 & $\begin{array}{c}40,1 \\
(266,1)\end{array}$ & 158 & $\begin{array}{c}20,0 \\
(133,1)\end{array}$ & 161 \\
\hline \multirow{3}{*}{ Eiche } & 1987 & $\begin{array}{c}20,3 \\
(240,0)\end{array}$ & 100 & $\begin{array}{c}11,4 \\
(135,0)\end{array}$ & 100 & $\begin{array}{c}5,7 \\
(67,0)\end{array}$ & 100 \\
\hline & 2002 & $\begin{array}{c}38,2 \\
(319,2)\end{array}$ & 188 & $\begin{array}{c}27,3 \\
(228,3)\end{array}$ & 239 & $\begin{array}{c}13,6 \\
(114,1)\end{array}$ & 238 \\
\hline & 2006 & $\begin{array}{c}33,1 \\
(276,8)\end{array}$ & 163 & $\begin{array}{c}23,1 \\
(192,9)\end{array}$ & 203 & $\begin{array}{c}11,5 \\
(96,4)\end{array}$ & 202 \\
\hline \multirow{3}{*}{ Fichte } & 1987 & $\begin{array}{c}47,7 \\
(228,0)\end{array}$ & 100 & $\begin{array}{c}18,0 \\
(86,0)\end{array}$ & 100 & $\begin{array}{c}9,0 \\
(43,0)\end{array}$ & 100 \\
\hline & 2002 & $\begin{array}{c}67,3 \\
(319,5)\end{array}$ & 141 & $\begin{array}{c}35,1 \\
(167,0)\end{array}$ & 195 & $\begin{array}{c}17,6 \\
(83,5)\end{array}$ & 195 \\
\hline & 2006 & $\begin{array}{c}70,6 \\
(334,7)\end{array}$ & 148 & $\begin{array}{c}36,5 \\
(173,4)\end{array}$ & 203 & $\begin{array}{c}18,2 \\
(86,7)\end{array}$ & 202 \\
\hline \multirow{3}{*}{ Kiefer } & 1987 & $\begin{array}{c}59,2 \\
(164,0)\end{array}$ & 100 & $\begin{array}{c}25,5 \\
(71,0)\end{array}$ & 100 & $\begin{array}{c}12,7 \\
(35,0)\end{array}$ & 100 \\
\hline & 2002 & $\begin{array}{c}79,5 \\
(243,1)\end{array}$ & 134 & $\begin{array}{c}51,4 \\
(157,2)\end{array}$ & 202 & $\begin{array}{c}25,7 \\
(78,6)\end{array}$ & 202 \\
\hline & 2006 & $\begin{array}{c}68,8 \\
(210,2)\end{array}$ & 116 & $\begin{array}{c}44,3 \\
(135,4)\end{array}$ & 174 & $\begin{array}{c}22,2 \\
(67,7)\end{array}$ & 175 \\
\hline
\end{tabular}


Der Gesamtderbholzvorrat der über die Altersklassen relativ gleichmäßig verteilten, aber flächenmäßig nur relativ schwach vertretenen Eichenbestände betrug 38,2 Mio. $\mathrm{m}^{3}$ (319,2 $\left.\mathrm{m}^{3} / \mathrm{ha}\right)$ mit einer lebenden Dendromasse von rund 27,3 Mio. $\mathrm{t}$ (228,3 t/ha), was einem Kohlenstoffvorrat von 13,6 Mio. t C (114,1 t C/ha) entspricht.

In den Fichtenwäldern mit ihrem Schwerpunkt in der II. und III. Altersklasse war ein Derbholzvorrat von 67,3 Mio. $\mathrm{m}^{3}$ vorhanden. Diesem Vorrat entspricht eine lebende Baumbiomasse von 35,1 Mio. $t$ bzw. ein C-Vorrat von ca. 17,6 Mio. t C. Die Durchschnittswerte beliefen sich auf $319,5 \mathrm{~m}^{3} /$ ha beim Derbholzvorrat, 158,9 t/ha bzw. 8,1 t/ha bei der lebenden ober- bzw. unterirdischen Biomasse sowie 83,5 t C/ha beim Kohlenstoffvorrat.

Die ebenfalls überwiegend der II. und III. Altersklasse zuzuordnenden Kiefernbestände wiesen einen Derbholzvorrat von insgesamt 79,5 Mio. $\mathrm{m}^{3}$ bzw. durchschnittlich 243,1 $\mathrm{m}^{3} /$ ha auf. Die gesamte lebende Baumbiomasse in Höhe von 51,4 Mio. t enthielt ca. 25,7 Mio. t C (s. Tab. 7). Das durchschnittliche Derbholzvolumen betrug $243,1 \mathrm{~m}^{3} /$ ha, die mittlere ober- bzw. unterirdische lebende Baumbiomasse 150,5 t/ha bzw. 6,7 t/ha und der mittlere Kohlenstoffvorrat 78,6 t C/ha.

Insgesamt fand in dem Zeitraum von 1987 bis 2002 bei allen Baumarten ein deutlicher Vorratsaufbau und damit auch eine entsprechende Zunahme der C-Vorräte statt (s. Tab. 7 u. Abb. 12), die bei den Nadelbaumarten mit ihrem hohen Anteil jüngerer Bestände ausgeprägter war als bei den altersklassenmäßig ausgeglichener aufgebauten Laubbaumbeständen. Unter den anderen Arten ist der hohe Anteil der Weichlaubbäume (ALn) (Birke, Erle, Pappel, Weide etc.), die überwiegend in den ersten drei Altersklassen vertreten sind (s. Abb. 4), hervorzuheben.

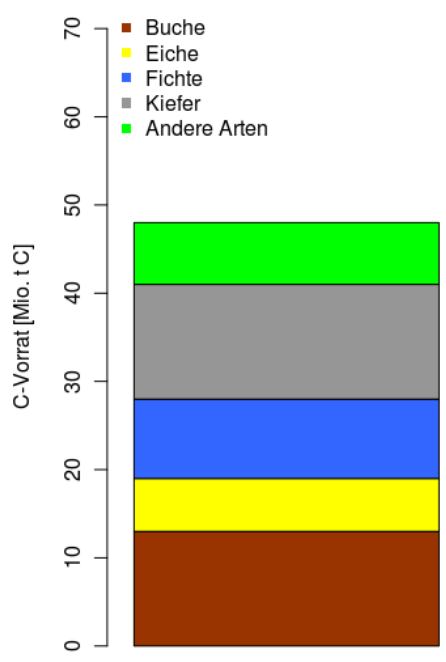

$\mathrm{BWI}^{1}$

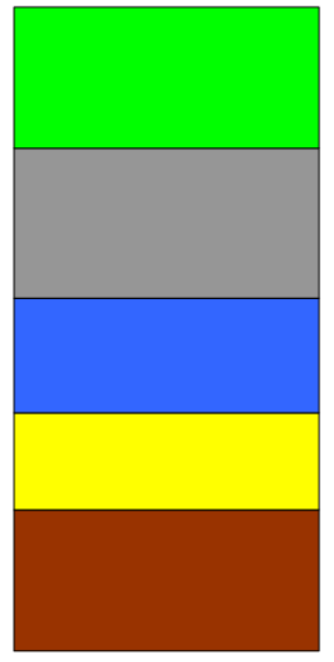

$B \mathrm{I}^{2}$
Abbildung 12:

C-Vorräte [Mio. tC] basierend auf den Derbholzvorräten der verschiedenen Baumarten in Niedersachsen zum Zeitpunkt der BWI ${ }^{1}$ (nicht funktional hergeleitet) und $B W I^{2}$

Beiträge aus der NW-FVA, Band 6, 2011 


\subsection{Fortschreibung bis Ende 2006}

\subsubsection{Lebende Baumbiomasse}

Von 2002 bis Ende 2006 fiel der Gesamtderbholzvorrat von 305 Mio. $^{3}$ auf 287 Mio. $\mathrm{m}^{3}$, die Durchschnittswerte von $279 \mathrm{~m}^{3} /$ ha auf $263 \mathrm{~m}^{3} /$ ha. Somit betrug der C-Vorrat im stehenden Derbholzvorrat 2006 für alle Baumarten 72 Mio. t C bzw. 66 t C/ha begehbare Holzbodenfläche.

Nach der Fortschreibung der Daten ins Jahr 2006 zeigten sich bei den Startwerten für die vier Hauptbaumarten der drei waldbaulichen Szenarien folgende Veränderungen: Die Derbholzvorräte für Buche und Fichte stiegen an, während sie für Eiche und Kiefer fielen. Ebenso haben sich die lebende Baumbiomasse und damit der C-Vorrat bei Buche und Fichte vergrößert respektive bei Eiche und Kiefer verringert (s. Tab. 7).

Die Betrachtung der Derbholzvorräte und der C-Vorräte der lebenden Baumbiomasse Ende 2006 nach 30- bzw. 40-jährigen Altersstufen verdeutlicht die verschiedenen Vorratsanteile und den unterschiedlichen Altersaufbau der Hauptbaumarten (s. Abb. 2, 4, 13 u. 14). Der zusätzliche Einfluss der unterschiedlichen Raumdichten der Baumarten wird bei den C-Vorräten deutlich. In der ersten Altersstufe (0-30 Jahre) sind die C-Vorräte der Nadelbaumarten höher als bei den Laubbaumarten. Insgesamt ist das Niveau jedoch niedrig, da viele Bestände noch nicht die Derbholzgrenze (BHD $\geq 7 \mathrm{~cm}$ ) überschritten haben. Deutlicher werden die Unterschiede in der zweiten Altersstufe (31-60 Jahre), in der die Masse der Nadelbaumbestände liegt. In der folgenden Altersstufe (61-100 Jahre) ist der CVorrat der Buche höher als der der Fichte, aber geringer als der der Kiefer. In der letzten Altersstufe (>100 Jahre) rücken die Laubbaumarten an die Spitze. Die Buche erreicht hier den höchsten mittleren C-Vorrat, gefolgt von der Eiche. 


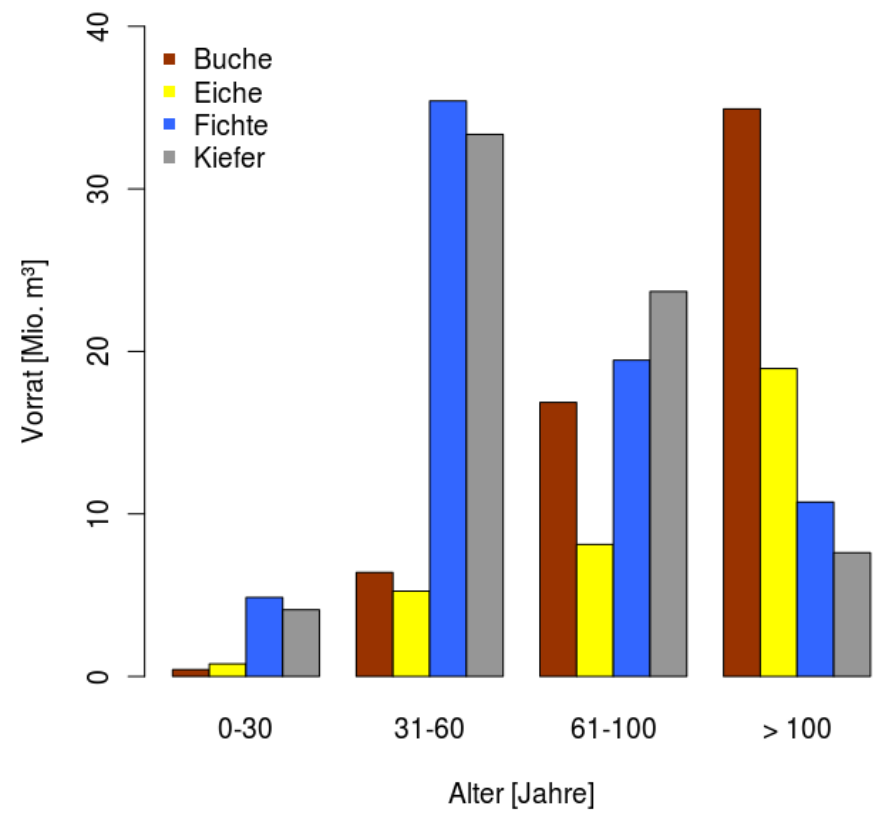

Abbildung 13: Derbholzvorräte [Mio. $\left.\mathrm{m}^{3}\right]$ der Hauptbaumarten nach Altersstufen Ende 2006

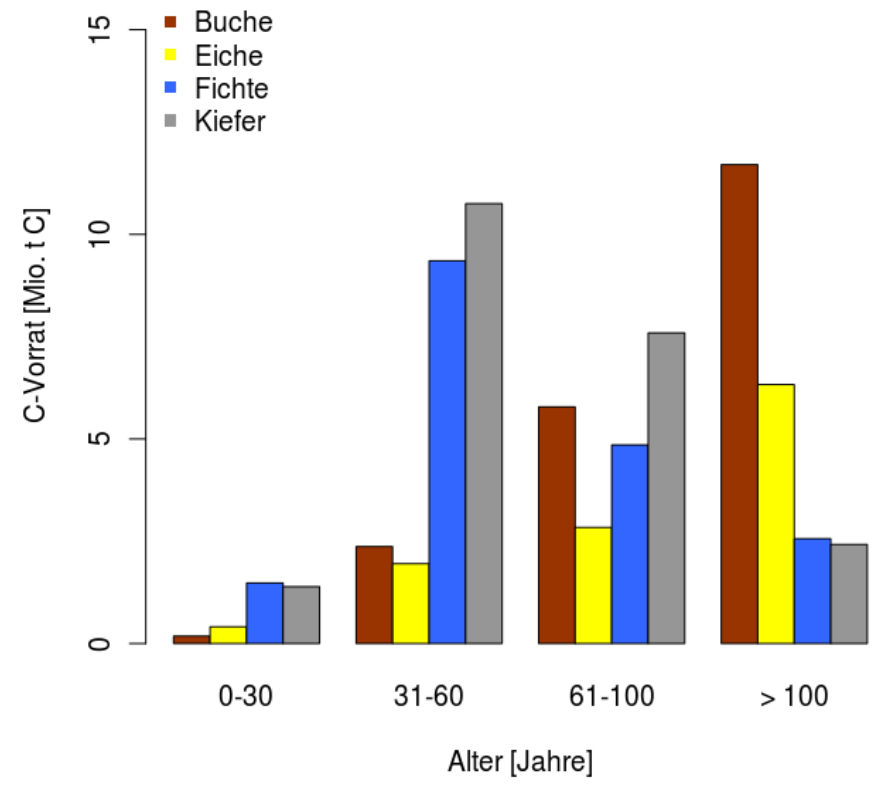

Abbildung 14: C-Vorräte [Mio. t C] der Hauptbaumarten nach Altersstufen Ende 2006 


\subsubsection{Tote Baumbiomasse}

Der Totholzvorrat in Niedersachsen belief sich zum Zeitpunkt der BWI $^{2}$ auf $8,1 \mathrm{~m}^{3} / \mathrm{ha}$ bzw. rund 8,9 Mio. $\mathrm{m}^{3}$ (ML 2004). Dieses Volumen wurde, wie in Kapitel 3.2.2 beschrieben, bis Ende 2006 fortgeschrieben. Dadurch stieg der Totholzvorrat auf 19,7 Mio. $\mathrm{m}^{3}(\mathrm{~d} \geq 7 \mathrm{~cm})$. Unterstellt man für dieses Volumen eine mittlere Raumdichte von $0,25 \mathrm{t} / \mathrm{m}^{3}$ und eine C-Konzentration von $50 \%$, so ergibt sich ein C-Vorrat in Höhe von 2,5 Mio. t C.

Theoretisch gehört die Wurzelbiomasse der genutzten Bäume ebenfalls zum Totholzpool. Da aber für dieses Kompartiment keine Funktionen zur Zersetzung im Erdreich vorliegen, können dazu keine speziellen Berechnungen durchgeführt werden. Im Folgenden ist daher immer von der oberirdischen toten Baumbiomasse die Rede.

\subsubsection{Nutzungen}

Durch die Vor- bzw. Endnutzung werden in der Fortschreibung den Beständen Massen und C-Vorräte entzogen. Die gesamte Nutzungsmasse im Zeitraum 2002 bis Ende 2006 betrug für alle Baumarten rund 19,6 Mio. Vfm, was in etwa 4,9 Mio. t C entspricht.

Betrachtet man die Hauptbaumarten, so waren bei der Eiche die Entnahmen deutlich geringer als bei der Buche. Die Endnutzungsmengen der Fichte sind überlagert durch die Kyrillschäden, die rechnerisch zum Startpunkt der Simulation vorgezogen wurden, und daher ungleich höher als bei den anderen Hauptbaumarten. Bei den Kiefernbeständen sind sie vergleichbar mit denjenigen der Buchenbestände. Den Fichtenbeständen wurde folglich bis Ende 2006 der größte C-Vorrat entnommen (s. Tab. 8).

Tabelle 8: Gesamte Vornutzungs- und Endnutzungsmengen sowie entnommener C-Vorrat von 2002 bis Ende 2006 inkl. der ,vorgezogenen “ Kyrillschäden für die analysierten Baumarten

\begin{tabular}{lccc}
\hline Baumart & $\begin{array}{c}\text { Vornutzung } \\
\text { [Mio. Vfm] }\end{array}$ & $\begin{array}{c}\text { Endnutzung } \\
\text { [Mio. Vfm] }\end{array}$ & $\begin{array}{c}\text { Entnommener } \\
\text { C-Vorrat } \\
{[\text { Mio. t C] }}\end{array}$ \\
\hline Buche & 2,5 & 0,6 & 0,9 \\
Eiche & 1,6 & 0,2 & 0,5 \\
Fichte & 2,3 & 4,4 & 1,3 \\
Kiefer & 3,1 & 1,3 & 0,9 \\
\hline
\end{tabular}




\subsubsection{Produktpool}

Die genannten Nutzungsmassen wurden dem Produktpool zugeführt. Dabei wurden die Baumarten zu Laub- oder Nadelholz zusammengefasst, um die jeweiligen Nutzungsmengen verschiedenen Holzverwendungen zuordnen zu können (s. Kap. 3.5).

Der Vorrat des Nadelholz-Produktpools erhöht sich in der kurzen Periode von 2002 bis Ende 2006 um das Doppelte im Vergleich zum Vorrat des LaubholzProduktpools. In diesen Holzmengen sind zu Beginn des Simulationszeitraumes ca. 1,37 Mio. t $\mathrm{C}$ in Produkten aus Laubholz und 2,22 Mio. t $\mathrm{C}$ in Produkten aus Nadelholz gespeichert (s. Tab. 9).

Tabelle 9: $\quad$ Akkumulierte C-Vorräte der Produktgruppen getrennt nach Laub-und Nadelholz.von 2002 bis Ende 2006

\begin{tabular}{lccccc}
\hline Art & $\begin{array}{c}\text { langer } \\
\text { Lebensdauer } \\
{[\text { Mio. t C }]}\end{array}$ & $\begin{array}{c}\text { Produkte mit } \\
\text { mittlerer } \\
\text { Lebensdauer } \\
{[\text { Mio. t C] }}\end{array}$ & $\begin{array}{c}\text { kurzer } \\
\text { Lebensdauer } \\
{[\text { Mio. t C }]}\end{array}$ & Energieholz & Summe \\
{$[$ Mio. t C $]$} & {$[$ Mio. t C] } \\
\hline Laubholz & 0,34 & 0,33 & 0,13 & 0,57 & 1,37 \\
Nadelholz & 0,77 & 0,52 & 0,62 & 0,31 & 2,22 \\
\hline
\end{tabular}

Die Aufteilung nach den vier Produktgruppen stellt sich beim Laubholz folgendermaßen dar: das Volumen der Produkte der Gruppe Energieholz wird auf 2,03 Mio. $\mathrm{m}^{3}$ geschätzt. Damit beträgt der C-Vorrat dieser Produktklasse 0,57 Mio. t C. Sehr viel geringer mit nur 0,46 Mio. $\mathrm{m}^{3}$ ist der Anteil der Produkte mit kurzer Lebensdauer am gesamten Produktpool. Daraus resultiert auch ein geringer C-Vorrat von 0,13 Mio.t C. Die Produkte mit mittlerer Lebensdauer erreichen ein geschätztes Volumen von 1,2 Mio. $\mathrm{m}^{3}$ und einen C-Vorrat von 0,33 Mio. t C. Ähnlich groß sind das Volumen (1,21 Mio. $\left.\mathrm{m}^{3}\right)$ und der C-Vorrat (0,34 Mio. t C) bei den Produkten mit langer Lebensdauer.

Die Produktklasse Energieholz hat beim Nadelholz, im Vergleich zum Laubholz, ein geringeres Volumen von 1,56 Mio. $\mathrm{m}^{3}$ mit einem C-Vorrat von 0,31 Mio. t C. Für die Produktklasse Produkte mit kurzer Lebensdauer werden 3,11 Mio. $\mathrm{m}^{3}$ berechnet, was einem C-Vorrat von 0,62 Mio. t C entspricht. Die Klasse Produkte mit mittlerer Lebensdauer hat ein Volumen von 2,61 Mio. $\mathrm{m}^{3}$, was einem C-Vorrat von rund 0,52 Mio. t gleichkommt. Das größte Volumen und der höchste C-Vorrat werden beim Nadelholz von Produkten mit langer Lebensdauer erreicht (3,87 Mio. $\mathrm{m}^{3}$ und 0,77 Mio. t C).

Die hier genannten Werte sind lediglich Schätzgrößen, die mit Hilfe der Holzproduktkategorien und des Holzverwendungsschlüssels (s. Tab. 5 und 6) aus den Nutzungsmengen der simulierten Fortschreibung, ergänzt um die Kyrill-Schad- 
holzmengen, abgeleitet wurden. Dabei sind Effekte, die durch Modifikationen in der Herstellung und Bearbeitung der Holzprodukte entstehen, nicht beachtet. Somit spiegeln die genannten Größen nur begrenzt die tatsächlichen C-Vorräte der Holzprodukte wider, die im Zeitraum 2002 bis Ende 2006 in den Zivilisationskreislauf eingingen. Sie sind eher als vorsichtige Näherungswerte zu betrachten, die sich auf das Rohholz beziehen und nicht die Effekte einer Kaskadennutzung abbilden (s. Kap. 3.5.2).

\subsection{Boden 2008}

Die C-Vorräte des Bodenpools werden getrennt nach Mineralböden sowie Mooren und anmoorigen Böden dargestellt. Für den Bodenpool wird nur der Zustand 2008, wie er sich durch die Analysen der BZE II-Daten für Niedersachsen ergibt, erörtert. Entwicklungen unter verschiedenen waldbaulichen Behandlungen bzw. Simulationsergebnisse können bisher noch nicht belastbar simuliert werden.

\subsubsection{Mineralböden}

Um den C-Vorrat der Streu zu ermitteln, wurden 149 Bodenprofile aus der BZE II genutzt. Davon befinden sich 93 Profile im niedersächsischen Tief- und 56 im niedersächsischen Bergland. Im Tiefland liegen 16 Probenpunkte in Laubwaldund 77 in Nadelwaldbeständen, die vorwiegend mit Kiefer bestockt sind. Im Bergland sind es 31 Stichprobenpunkte in Beständen aus Laubbaumarten, meist Buche, und 25 aus Nadelbaumarten, überwiegend Fichte. Diese Profile haben differenzierte mittlere C-Vorräte in der Humusauflage. Bei einer Betrachtung getrennt nach Regionen ergibt sich folgendes Bild: In der Humusauflage der Laubwaldbestände im Tiefland beträgt der mittlere $\mathrm{C}$-Vorrat $17 \mathrm{tC} / \mathrm{ha}$, im Bergland $7 \mathrm{t} \mathrm{C} /$ ha sowie insgesamt im Mittel $10 \mathrm{t} \mathrm{C/ha.} \mathrm{In} \mathrm{den} \mathrm{Nadelwaldbeständen} \mathrm{über-}$ trifft der C-Vorrat der Auflage generell denjenigen der Laubwaldbestände um $18 \mathrm{t} \mathrm{C} / \mathrm{ha}$, liegt also im Tiefland bei $35 \mathrm{t} \mathrm{C} / \mathrm{ha}$ und im Bergland bei $25 \mathrm{t} \mathrm{C} / \mathrm{ha}$. Die mittlere Kohlenstoffspeicherung im Auflagehumus der Nadelbaumbestände beträgt in Niedersachsen rund $32 \mathrm{t} C /$ ha. Das niedersächsische Mittel für den CVorrat im Auflagehumus liegt bei rund $25 \mathrm{t} \mathrm{C} / \mathrm{ha}$.

Beim C-Vorrat der Humusauflage zeigt sich, dass er maßgeblich vom aufstockenden Bestand, insbesondere dem Bestandesalter, der Bestandesdichte, dem Anteil der Nadelbaumarten und der Region (Berg- oder Tiefland) beeinflusst wird.

Der C-Vorrat des Mineralbodens basiert auf einer vorläufigen Auswertung von 27 Bodenprofilproben der BZE II-Punkte. Davon befinden sich 11 im niedersächsischen Bergland und 16 im Tiefland. Der mittlere C-Vorrat dieser Profile liegt im Bergland bei ca. 79 t C/ha und im Tiefland bei ca. 103 t C/ha. Für Laub- bzw. Nadelwaldbestände beträgt der C-Vorrat ohne Aufteilung der Profile nach Regionen 72 t C/ha bzw. 102 t C/ha. 
Ein Vergleich der Gesamtvorräte von Auflage und Mineralboden mit den Werten der lebenden Baumbiomasse aus dem Jahr 2006 zeigt, dass in Laubwaldbeständen das Verhältnis rund 1:1 und in Nadelwaldbeständen bis zu 2:1 beträgt. Dies bedeutet, dass der Boden in Nadelwaldbeständen doppelt so viel C speichern kann wie deren oberirdische lebende Baumbiomasse.

\subsubsection{Moore und anmoorige Böden}

Moore nehmen in den Wäldern Niedersachsens eine Fläche von ca. 12.000 ha ein (ca. $1 \%$ der Waldfläche), von denen $26 \%$ im Berg- und $74 \%$ im Tiefland liegen. Diese Fläche wird durch 13 Profile der BZE II repräsentiert, wobei nur ein Profil im Bergland aufgenommen wurde. Auf eine Aufteilung der Daten nach Regionen wurde daher verzichtet. Insgesamt befinden sich 5 Profile in Laub- und 8 Profile in Nadelwaldbeständen.

Der mittlere C-Vorrat der Auflagen in Mooren und bei anmoorigen Böden beläuft sich in Beständen aus Laub- bzw. Nadelbaumarten auf $29 \mathrm{t} \mathrm{C/ha}$ bzw. 36 t C/ha.

Da die Streuung der Daten im Nadelwald sehr viel größer als im Laubwald ist und um die Datengrundlage zu verbreitern, wurden die Daten zusammengefasst. Danach ergibt sich für Auflage und Oberboden bis $90 \mathrm{~cm}$ Tiefe der Moore und anmoorigen Böden in Niedersachsen ein mittlerer C-Vorrat von rund $457 \mathrm{t} \mathrm{C} / \mathrm{ha}$. Dieser Wert ist ca. viermal höher als bei den mineralischen Böden und unterstreicht damit die Bedeutung der C-Speicherung in Mooren und anmoorigen Böden.

\subsection{Simulation von 2007 bis 2036 - Szenarienvergleich}

\subsubsection{Entwicklung der Derbholzvorräte, der lebenden Baumbiomassen und deren C- Vorräte}

Die simulierten Szenarien ertragsorientierter, naturnaher und naturschutzorientierter Waldbau haben einen großen Einfluss auf die betrachteten Kompartimente Derbholzvorrat, lebende Baumbiomasse und deren C-Vorrat.

Durch das naturnahe Waldbauszenario steigt der Derbholzvorrat des Gesamtwaldes bis zum Jahr 2036 im Vergleich zu 2006 leicht an (auf 288 Mio. m³). Somit ist der C-Vorrat auch ähnlich hoch wie zu Beginn der Simulation (72 Mio. t C). Den stärksten Vorratsaufbau verzeichnet das naturschutzorientierte Szenario. Dort steigt der Derbholzvorrat bis 2036 auf 380 Mio. $\mathrm{m}^{3}$ an, wodurch sich auch der darin gespeicherte C-Vorrat für alle Baumarten auf 95 Mio. t C erhöht. Einen anderen Trend kann man bei der ertragsorientierten Variante beobachten. Dort sinkt der Gesamtderbholzvorrat bis zum Jahr 2036 auf 218 Mio. $\mathrm{m}^{3}$ und der darin 
enthaltene C-Vorrat auf rund 55 Mio. t C ab. Die Veränderungen der gesamten Derbholzvorräte und der in ihnen gespeicherten C-Gehalte von der BWI 1987 bis zum Ende der Simulationsrechnungen 2036 zeigt Abbildung 15.

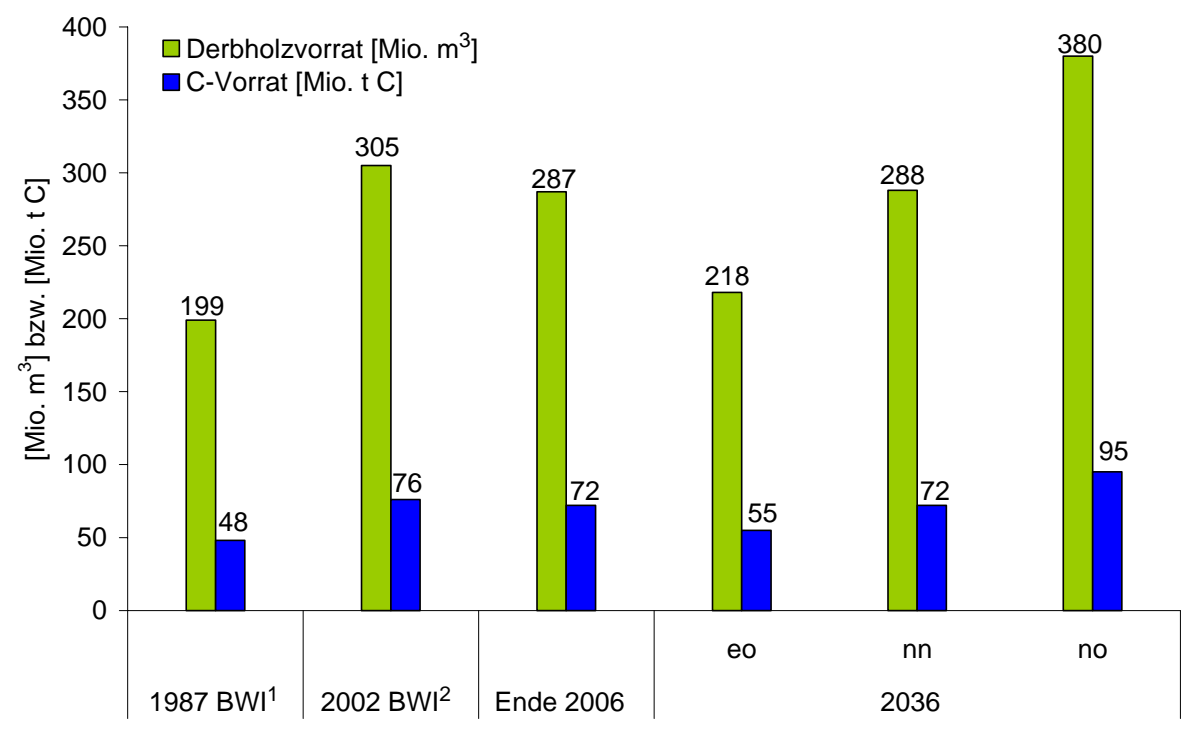

Abbildung 15: Veränderung der gesamten Derbholzvorräte und der in ibnen gespeicherten C-Gehalte von der BWI 1987 bis zum Ende des Simulationszeitraumes 2036 (eo = ertragsorientiertes, $n n=$ naturnahes, $n_{0}=$ naturschutzorientiertes $W$ aldbauszenario)

In den Abbildungen 16 bis 18 wird die Entwicklung der Derbholzvorräte, Baumbiomassen und C-Vorräte von Ende 2006 bis Ende 2036 für die drei unterschiedlichen Szenarien und die vier untersuchten Baumarten dargestellt. Die z. T. anfänglich steileren Abfälle der Kurven erklären sich dadurch, dass ungeachtet der Besitzart und der Vorgeschichte der Bestände alle Stichprobenpunkte in der Simulation regelkonform behandelt und zunächst auf die Vorgaben der Szenarien eingesteuert werden.

Im ertragsorientierten Szenario mit seinen stärkeren Eingriffen und geringeren Zielstärken nehmen die Gesamtderbholzvorräte der Baumarten Buche, Fichte und Kiefer kontinuierlich ab. In den Eichenbeständen fällt der Derbholzvorrat bis 2021, um dann wieder leicht anzusteigen (s. Abb. 16a). Die gleichen Trends zeigen sich bei den Biomassen der Hauptbaumarten und den C-Vorräten (s. Abb. 17a und 18a). Die relativen Veränderungen sind bei den Laubbaumarten geringer als bei den Nadelbaumarten (s. Tab. 10).

Beim naturnahen Bewirtschaftungsszenario sinken bis zum Ende des 30-jährigen Simulationszeitraumes die Derbholzvorräte, Baumbiomassen und C-Vorräte bei allen Baumarten. Zwischenzeitlich kommt es zu mehr oder weniger deutlichen 
Abnahmen bzw. leichten Anstiegen, die im Zusammenhang mit dem unterschiedlichen Altersklassenaufbau der Hauptbaumarten zu sehen sind (s. Abb. 4). Die Derbholzvorräte reichen von anfangs 33 Mio. Vfm bei Eiche bis zu 70 Mio. Vfm bei Fichte und liegen nach 30 Jahren zwischen 32 Mio. Vfm bei Eiche und 67 Mio. Vfm bei Kiefer (s. Abb. 16b). Aufgrund der unterschiedlichen Raumdichten rücken bei der lebenden Baumbiomasse die Laub- bzw. Nadelbaumarten näher zusammen (s. Abb. 17b). Die C-Vorräte in der lebenden Baumbiomasse bewegen sich anfangs zwischen 20,04 Mio. t $\mathrm{C}$ bei Buche, 11,53 Mio. t $\mathrm{C}$ bei Eiche, 18,25 Mio. t $\mathrm{C}$ bei Fichte und 22,16 Mio.t $\mathrm{C}$ bei Kiefer und verringern sich zwischen 2006 und 2036 um $4 \%$ (Kiefer) bis $16 \%$ (Fichte) (s. Abb. 18b und Tab. 10).

Durch die höheren Naturschutzstandards, schwächeren Eingriffe und höheren Zielstärken nehmen bei dem naturschutzorientierten Szenario die Derbholzvorräte aller Baumarten zu (s. Abb. 16c). Ebenso steigen die Biomassen in der Zeit von 2011 bis 2036 unterschiedlich stark an, wobei sich die Buche und die Nadelbaumarten deutlich von der Eiche absetzen (s. Abb. 17c). Der darin gespeicherte C-Vorrat erhöht sich gleichgerichtet (s. Abb. 18c). Die relativen Zunahmen sind bei Buche (+ $24 \%$ ) und Fichte (+ $34 \%$ ) am größten (s. Tab. 10). 
a) eo

b) $\mathrm{nn}$
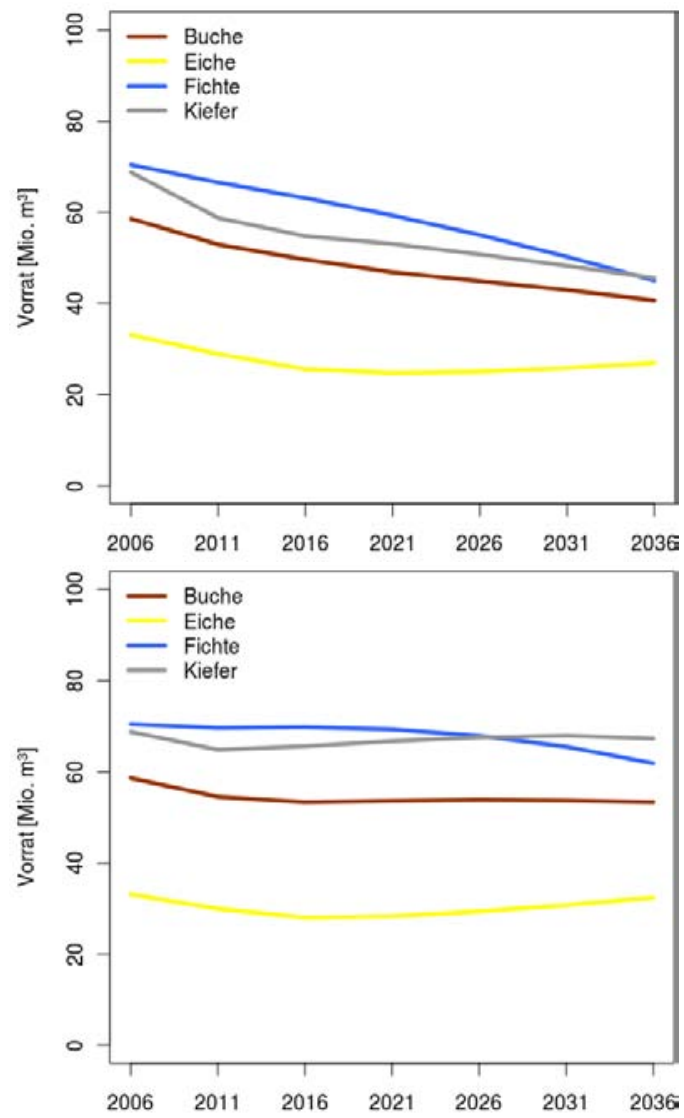

c) no

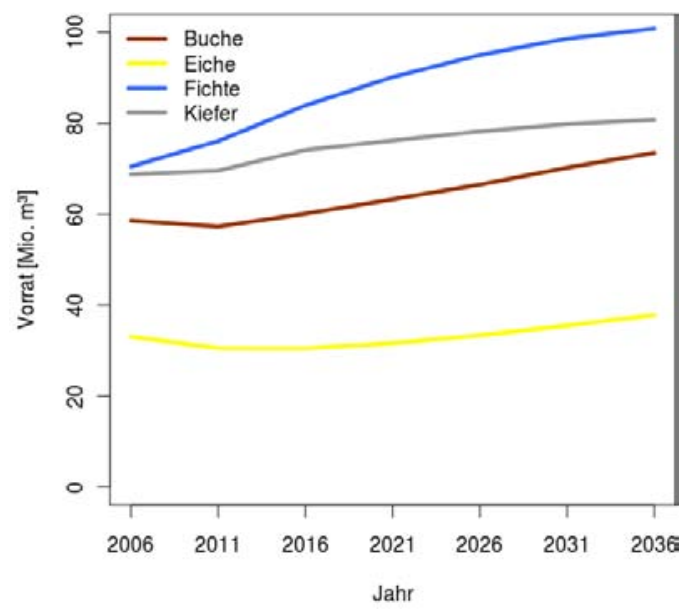

Abbildung 16: Entwicklung der Derbholzvorräte [Mio. $\mathrm{m}^{3}$ ] nach Hauptbaumarten für die Szenarien a) ertragsorientierter $W$ aldbau (eo), b) naturnaher $W$ aldbau (nn) und c) naturschutzorientierter Waldbau (no) 
a) eo

b) $\mathrm{nn}$
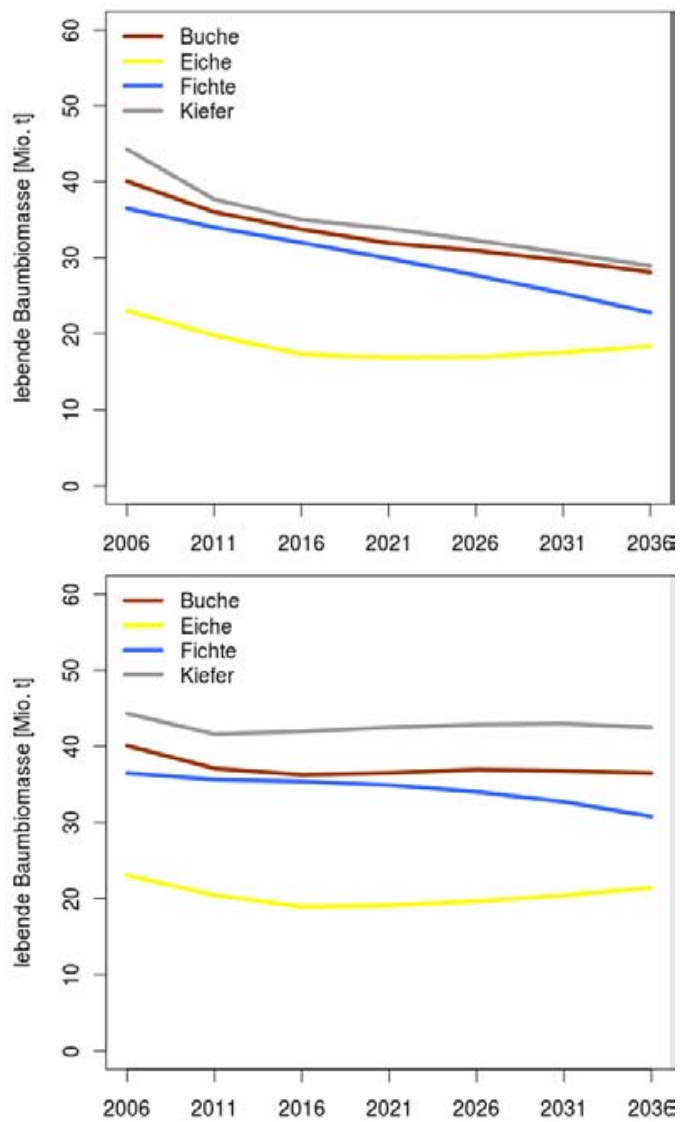

c) no

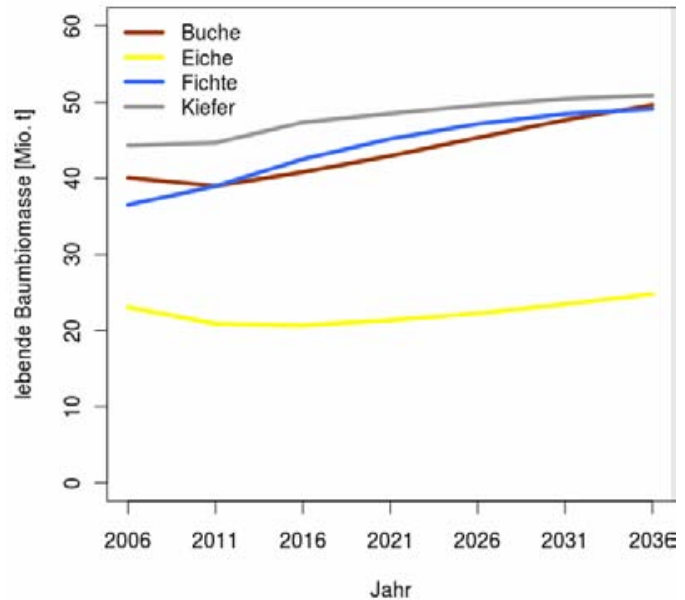

Abbildung 17: Entwicklung der lebenden Baumbiomasse [Mio. t] nach Hauptbaumarten für die Szenarien a) ertragsorientierter $W$ aldbau (eo), b) naturnaher $W$ aldbau (nn) und c) naturschutzorientierter Waldbau (no)

Beiträge aus der NW-FVA, Band 6, 2011 
a) eo

b) $\mathrm{nn}$
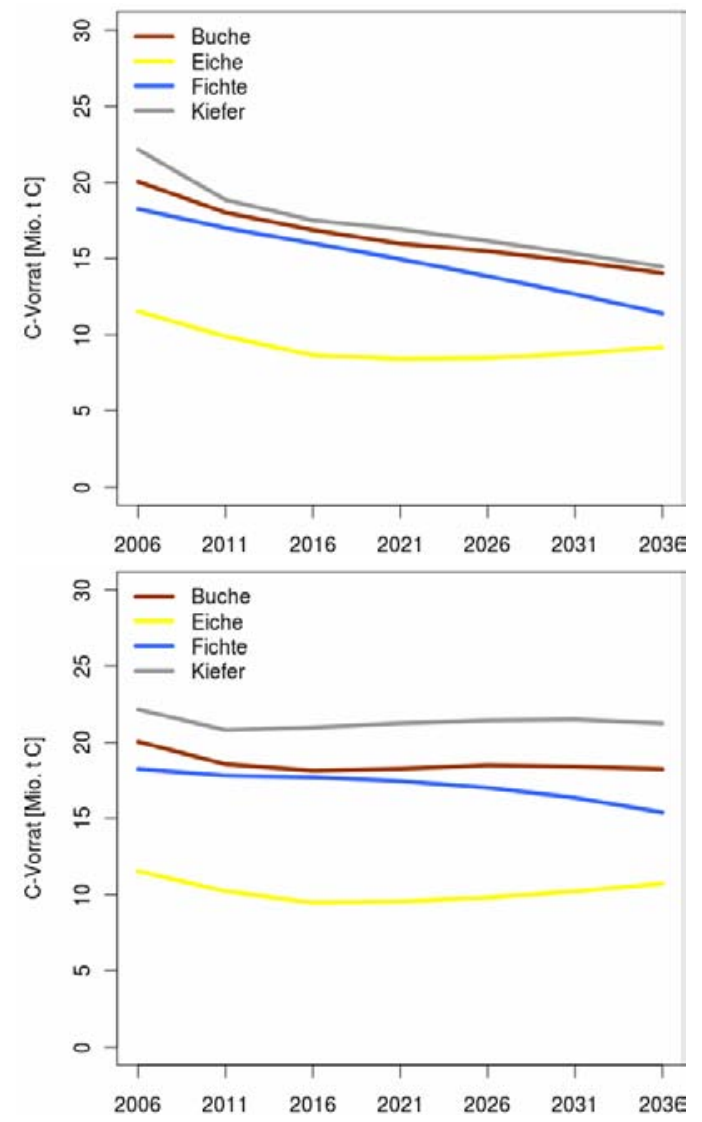

c) no

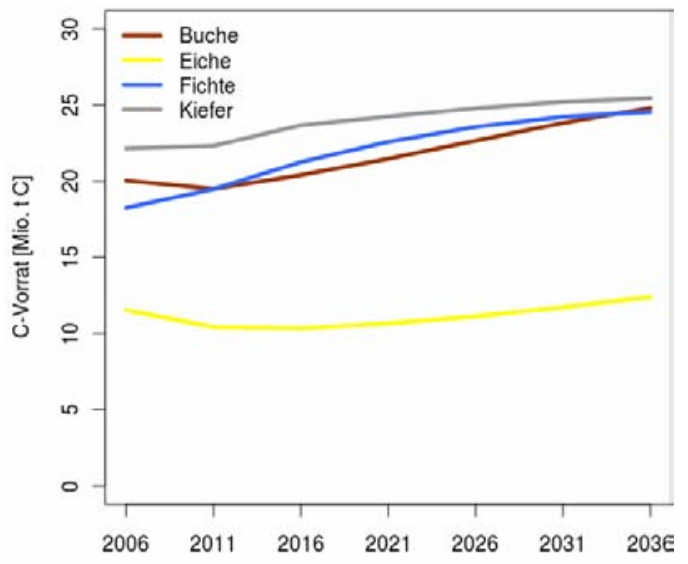

Abbildung 18: Entwicklung der C-Vorräte [Mio. $t C$ ] der lebenden Baumbiomasse nach Hauptbaumarten für die Szenarien a) ertragsorientierter $W$ aldbau (eo), b) naturnaher $W$ aldbau (nn) und c) naturschutzorientierter W aldbau (no) 
Tabelle 10: Derbholzvorrat (DV), lebende Baumbiomasse (LBM) und C-Vorrat (CV) der untersuchten Hauptbaumarten zu Beginn und am Ende des dreißigjährigen Simulationszeitraumes getrennt für die Szenarien ertragsorientierter, naturnaher und naturschutzorientierter Waldbau - in Klammern die relativen Veränderungen (Ausgangszustand Ende 2006: $100 \%$ )

\begin{tabular}{|c|c|c|c|c|c|}
\hline \multirow[t]{2}{*}{ Baumart } & \multirow[t]{2}{*}{ Kompartiment } & \multirow[t]{2}{*}{$\begin{array}{l}\text { Ausgangs- } \\
\text { zustand } \\
\text { (Ende 2006) }\end{array}$} & \multicolumn{3}{|c|}{$\begin{array}{c}\text { Endzustand } \\
\text { (2036) }\end{array}$} \\
\hline & & & $\begin{array}{l}\text { ertrags- } \\
\text { orientiert }\end{array}$ & naturnah & $\begin{array}{l}\text { naturschutz- } \\
\text { orientiert }\end{array}$ \\
\hline \multirow{3}{*}{ Buche } & $\begin{array}{c}\text { DV } \\
{\left[\text { Mio. } \mathrm{m}^{3}\right]}\end{array}$ & $\begin{array}{c}58,61 \\
(100 \%)\end{array}$ & $\begin{array}{c}40,69 \\
(-30 \%)\end{array}$ & $\begin{array}{c}53,34 \\
(-9 \%)\end{array}$ & $\begin{array}{c}73,49 \\
(+25 \%)\end{array}$ \\
\hline & $\begin{array}{c}\text { LBM } \\
{[\text { Mio. t] }}\end{array}$ & $\begin{array}{c}40,08 \\
(100 \%)\end{array}$ & $\begin{array}{c}28,09 \\
(-30 \%)\end{array}$ & $\begin{array}{l}36,49 \\
(-8 \%)\end{array}$ & $\begin{array}{c}49,64 \\
(+24 \%)\end{array}$ \\
\hline & $\begin{array}{c}\text { CV } \\
{[\text { Mio. t C] }}\end{array}$ & $\begin{array}{c}20,04 \\
(100 \%)\end{array}$ & $\begin{array}{c}14,05 \\
(-30 \%)\end{array}$ & $\begin{array}{l}18,24 \\
(-9 \%)\end{array}$ & $\begin{array}{c}24,82 \\
(+24 \%)\end{array}$ \\
\hline \multirow{3}{*}{ Eiche } & $\begin{array}{c}\text { DV } \\
{\left[\text { Mio. } \mathrm{m}^{3}\right]}\end{array}$ & $\begin{array}{c}33,10 \\
(100 \%)\end{array}$ & $\begin{array}{c}26,94 \\
(-18 \%)\end{array}$ & $\begin{array}{l}32,40 \\
(-2 \%)\end{array}$ & $\begin{array}{l}37,81 \\
(+14)\end{array}$ \\
\hline & $\begin{array}{l}\text { LBM } \\
{[\text { Mio. t] }}\end{array}$ & $\begin{array}{c}23,06 \\
(100 \%)\end{array}$ & $\begin{array}{c}18,38 \\
(-20 \%)\end{array}$ & $\begin{array}{c}21,44 \\
(-7 \%)\end{array}$ & $\begin{array}{c}24,79 \\
(+7 \%)\end{array}$ \\
\hline & $\begin{array}{c}\text { CV } \\
{[\text { Mio. t C] }}\end{array}$ & $\begin{array}{c}11,53 \\
(100 \%)\end{array}$ & $\begin{array}{c}9,19 \\
(-20 \%)\end{array}$ & $\begin{array}{c}10,72 \\
(-7 \%)\end{array}$ & $\begin{array}{c}12,40 \\
(+8 \%)\end{array}$ \\
\hline \multirow{3}{*}{ Fichte } & $\begin{array}{c}\text { DV } \\
{\left[\text { Mio. } \mathrm{m}^{3}\right]}\end{array}$ & $\begin{array}{c}70,46 \\
(100 \%)\end{array}$ & $\begin{array}{c}45,02 \\
(-36 \%)\end{array}$ & $\begin{array}{c}61,88 \\
(-12 \%)\end{array}$ & $\begin{array}{c}100,82 \\
(+43 \%)\end{array}$ \\
\hline & $\begin{array}{c}\text { LBM } \\
{[\text { Mio. t] }}\end{array}$ & $\begin{array}{c}36,50 \\
(100 \%)\end{array}$ & $\begin{array}{c}22,79 \\
(-37 \%)\end{array}$ & $\begin{array}{c}30,79 \\
(-16 \%)\end{array}$ & $\begin{array}{c}49,10 \\
(+34 \%)\end{array}$ \\
\hline & $\begin{array}{c}\text { CV } \\
{[\text { Mio. t C] }}\end{array}$ & $\begin{array}{c}18,25 \\
(100 \%)\end{array}$ & $\begin{array}{c}11,39 \\
(-38 \%)\end{array}$ & $\begin{array}{c}15,39 \\
(-16 \%)\end{array}$ & $\begin{array}{c}24,55 \\
(+34 \%)\end{array}$ \\
\hline \multirow{3}{*}{ Kiefer } & $\begin{array}{c}\text { DV } \\
{\left[\text { Mio. } \mathrm{m}^{3}\right]}\end{array}$ & $\begin{array}{c}68,76 \\
(100 \%)\end{array}$ & $\begin{array}{c}45,62 \\
(-36 \%)\end{array}$ & $\begin{array}{c}67,30 \\
(-2 \%)\end{array}$ & $\begin{array}{c}80,76 \\
(+17 \%)\end{array}$ \\
\hline & $\begin{array}{c}\text { LBM } \\
{[\text { Mio. t] }}\end{array}$ & $\begin{array}{c}44,31 \\
(100 \%)\end{array}$ & $\begin{array}{c}28,94 \\
(-35 \%)\end{array}$ & $\begin{array}{l}42,49 \\
(-4 \%)\end{array}$ & $\begin{array}{c}50,86 \\
(+15 \%)\end{array}$ \\
\hline & $\begin{array}{c}\mathrm{CV} \\
{[\text { Mio. t C] }}\end{array}$ & $\begin{array}{c}22,16 \\
(100 \%)\end{array}$ & $\begin{array}{c}14,47 \\
(-35 \%)\end{array}$ & $\begin{array}{l}21,24 \\
(-4 \%)\end{array}$ & $\begin{array}{c}25,43 \\
(+15 \%)\end{array}$ \\
\hline
\end{tabular}




\subsubsection{Nutzungen}

Durch die Vor- und Endnutzungen wird den Beständen Biomasse und damit Kohlenstoff entzogen, der überwiegend dem Holzproduktepool zugeführt wird.

Tabelle 11: Akkumulierte Nutzungsmengen (N) und entnommene Massen (M) sowie darin enthaltene C-Vorräte (CV) der verschiedenen waldbaulichen Szenarien von Ende 2006 bis 2036 für die Hauptbaumarten

\begin{tabular}{|c|c|c|c|c|}
\hline \multirow[t]{2}{*}{ Baumart } & \multirow[t]{2}{*}{ Kompartiment } & \multicolumn{3}{|c|}{$\begin{array}{c}\text { Endzustand } \\
\text { (2036) }\end{array}$} \\
\hline & & ertragsorientiert & naturnah & $\begin{array}{c}\text { naturschutz- } \\
\text { orientiert }\end{array}$ \\
\hline \multirow{3}{*}{ Buche } & $\begin{array}{c}\mathrm{N} \\
{\left[\text { Mio. } \mathrm{m}^{3}\right]}\end{array}$ & 70,0 & 63,4 & 44,5 \\
\hline & $\begin{array}{c}\text { M } \\
{[\text { Mio. } t]}\end{array}$ & 38,8 & 35,1 & 24,6 \\
\hline & $\begin{array}{c}\text { CV } \\
\text { [Mio. t C] }\end{array}$ & 19,4 & 17,6 & 12,3 \\
\hline \multirow{3}{*}{ Eiche } & $\begin{array}{c}\mathrm{N} \\
{\left[\text { Mio. } \mathrm{m}^{3}\right]}\end{array}$ & 28,7 & 24,3 & 20,1 \\
\hline & $\begin{array}{c}\mathrm{M} \\
\text { [Mio. t] }\end{array}$ & 16,1 & 13,7 & 11,2 \\
\hline & $\begin{array}{c}\mathrm{CV} \\
{[\text { Mio. t C] }}\end{array}$ & 8,0 & 6,8 & 5,6 \\
\hline \multirow{3}{*}{ Fichte } & $\begin{array}{c}\mathrm{N} \\
{\left[\text { Mio. } \mathrm{m}^{3}\right]}\end{array}$ & 100,3 & 95,4 & 60,0 \\
\hline & $\begin{array}{c}\mathrm{M} \\
\text { [Mio. t] }\end{array}$ & 37,8 & 36,0 & 22,6 \\
\hline & $\begin{array}{c}\text { CV } \\
\text { [Mio. t C] }\end{array}$ & 18,9 & 18,0 & 11,3 \\
\hline \multirow{3}{*}{ Kiefer } & $\begin{array}{c}\mathrm{N} \\
{\left[\text { Mio. } \mathrm{m}^{3}\right]}\end{array}$ & 64,3 & 46,5 & 32,0 \\
\hline & $\begin{array}{c}\mathrm{M} \\
\text { [Mio. t] }\end{array}$ & 27,7 & 20,0 & 13,8 \\
\hline & $\begin{array}{c}\text { CV } \\
\text { [Mio. t C] }\end{array}$ & 13,8 & 10,0 & 6,9 \\
\hline
\end{tabular}

Bezogen auf den gesamten niedersächsischen Wald und alle Baumarten werden in dem dreißigjährigen Simulationszeitraum bei der ertragsorientierten Variante rund 
352 Mio. $\mathrm{m}^{3}$, bei der naturnahen Variante ca. 295 Mio. $\mathrm{m}^{3}$ und bei der naturschutzorientierten Variante etwa 201 Mio. $\mathrm{m}^{3}$ Derbholz geerntet. Das hat einen Kohlenstoffentzug aus dem Wald in Höhe von ca. 88 Mio. t C beim ertragsorientierten Szenario, von 74 Mio. t $C$ beim naturnahen Szenario und von 50 Mio. t C beim naturschutzorientierten Szenario zur Folge.

Betrachtet man die Hauptbaumarten, so werden nach dem Szenario naturnaher Waldbau bei Buche und Fichte deutlich höhere Einschläge realisiert als bei Eiche und Kiefer. Dementsprechend sind auch die Massen und C-Vorräte der Nutzungen bei Buche und Fichte höher. Die schwächeren Nutzungsintensitäten des naturschutzorientierten Konzeptes bewirken geringere, die stärkeren Nutzungsintensitäten des ertragsorientierten Szenarios höhere Entzüge an C-Vorräten im 30-jährigen Simulationszeitraum im Vergleich zum naturnahen Bewirtschaftungskonzept (s. Tab. 11).

\subsubsection{Tote Baumbiomasse}

Die drei waldbaulichen Szenarien führen im 30-jährigen Simulationszeitraum zu einer mehr oder weniger deutlichen Erhöhung bzw. Verringerung der Totholzvorräte bei den Hauptbaumarten. Die Unterschiede ergeben sich durch die unterschiedlichen Mengen der Erntereste je nach Eingriffsstärke, -häufigkeit und Baumart und den schrittweisen Anreicherungen (vgl. angestrebte Totholzvorräte in Tab. 1) sowie aus den baumartenspezifischen Zersetzungsraten. Gleiches gilt für die in diesem Pool enthaltenen C-Vorräte (s. Abb. 19 bis 22).

Im naturnahen Szenario sind die relativen Änderungen der C-Vorräte beim Totholz am größten für Buche und Fichte. Bei der Eiche nehmen die C-Vorräte im Totholz leicht ab, während sie bei der Kiefer etwas ansteigen. Unter den waldbaulichen Szenarien nimmt das naturnahe Szenario hinsichtlich der C-Vorräte im Totholz und ihren Änderungen eine Mittelstellung ein (s. Tab. 12).

Beim naturschutzorientierten Waldbauszenario werden zwischen 2007 und 2036 die größten Abnahmen für die Buche, aber deutliche Zunahmen für die Kiefer in den C-Vorräten des Totholzpools geschätzt. Die Werte für die Kiefer sind hier im Vergleich der Szenarien auffallend hoch (s. Tab. 12).

Die relative Verringerung der C-Vorräte in der toten Baumbiomasse der Nadelhölzer erreicht beim ertragsorientierten Szenario die höchsten Werte. Für Buche ist sie im Vergleich zur naturnahen Variante gleich und für Eiche nur etwas höher. Der C-Vorrat im Totholzspeicher hat bei diesem Szenario für Fichte und Kiefer am stärksten abgenommen (s. Tab. 12 u. Abb. 19 bis 22).

Unabhängig von den Behandlungsvarianten fallen die großen relativen Veränderungen bei Buche und Fichte und die starke Zunahme im naturschutzorientierten Szenario bei der Kiefer auf. Dies ist vor allem im Zusammenhang mit den Unterschieden in den Nutzungsmengen zu sehen (s. Kap. 4.3.2). 


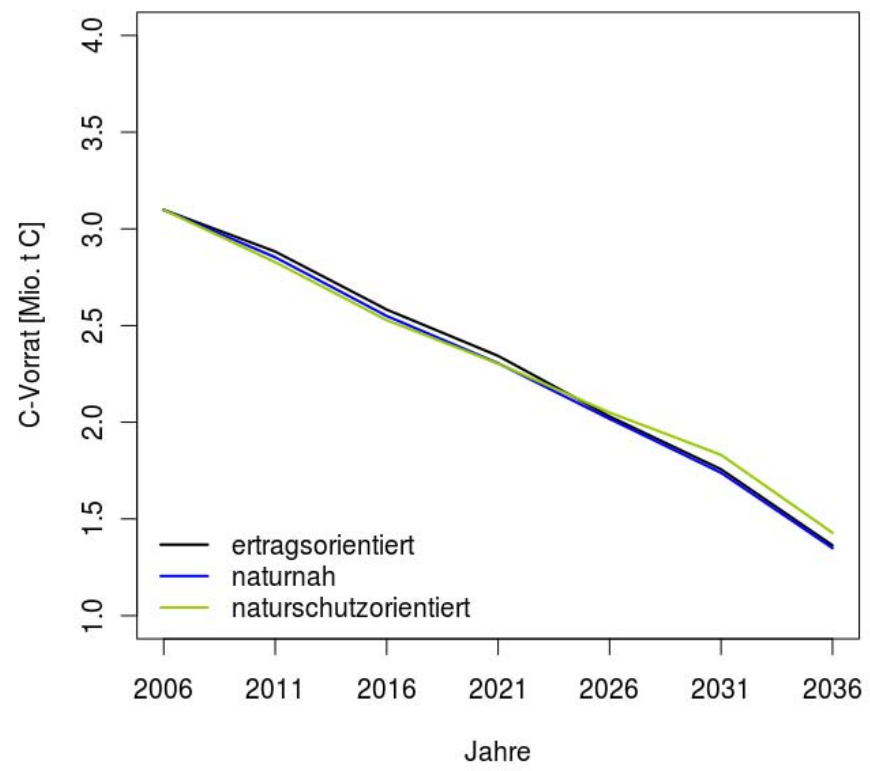

Abbildung 19: Entwicklung des C-Vorrates [Mio. tC] im Totholzspeicher für Buche bei den verschiedenen waldbaulichen $S$ zenarien

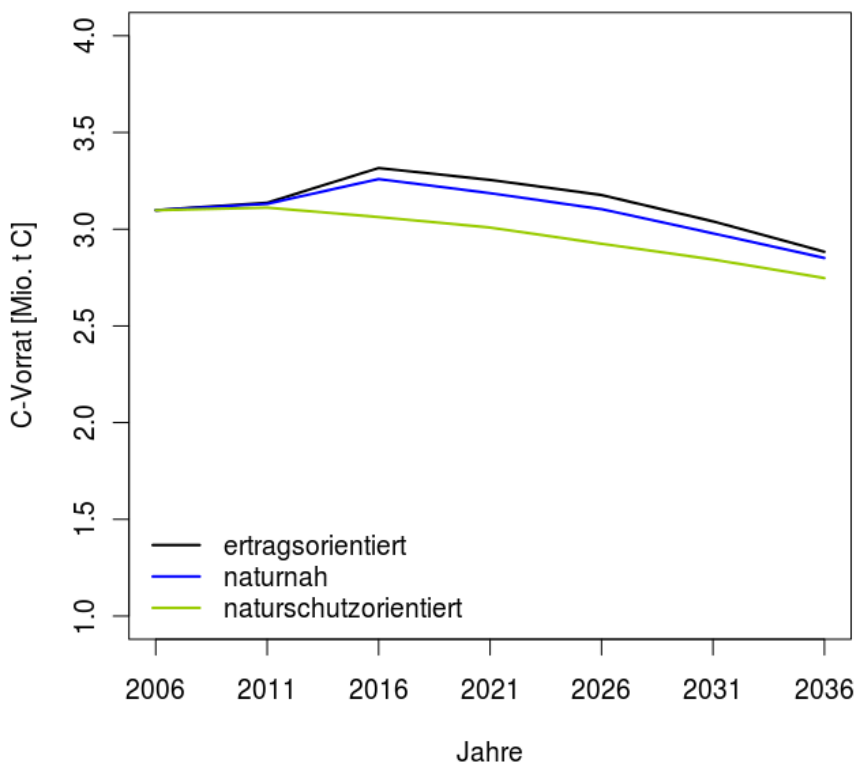

Abbildung 20: Entwicklung des C-Vorrates [Mio. tC] im Totholzspeicher für Eiche bei den verschiedenen waldbaulichen $S$ zenarien 


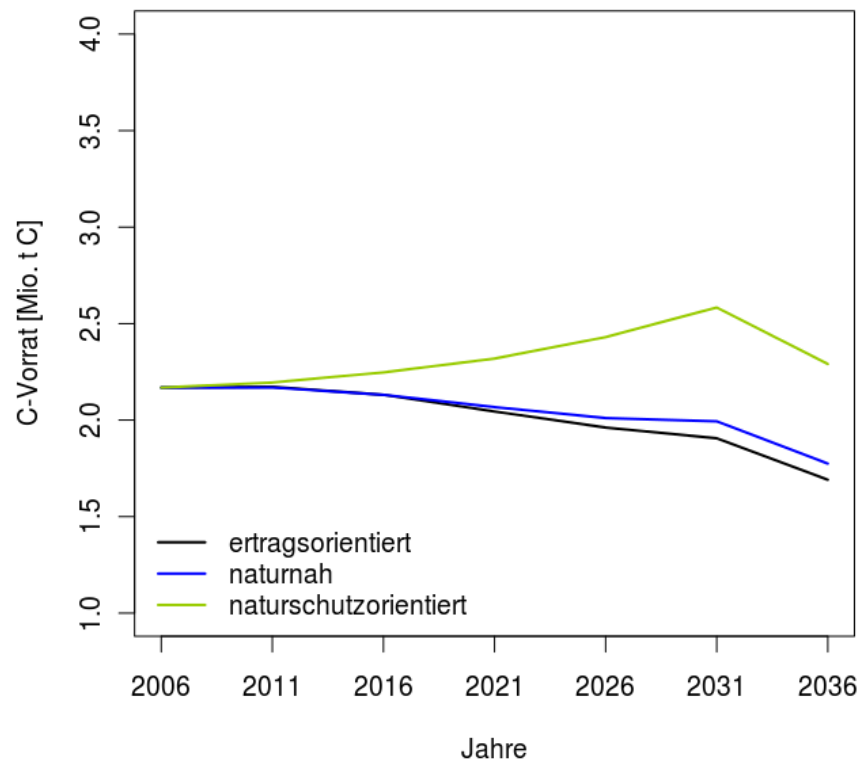

Abbildung 21: Entwicklung des C-Vorrates [Mio. t C] im Totholzspeicher für Fichte bei den verschiedenen waldbaulichen $S_{\text {zenarien }}$

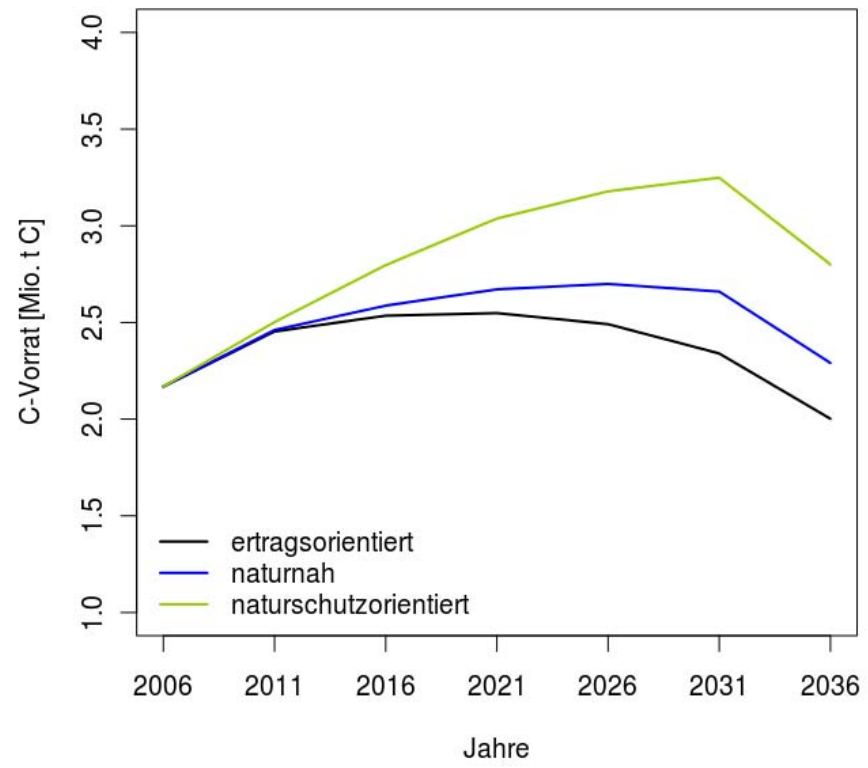

Abbildung 22: Entwicklung des C-Vorrates [Mio. t C] im Totholzspeicher für Kiefer bei den verschiedenen waldbaulichen $S_{\text {zenarien }}$ 
Tabelle 12: C-Vorrat [Mio. tC] im Totholzspeicher 2036 getrennt für die drei waldbaulichen Szenarien und die untersuchten Hauptbaumarten - in Klammern die relative Veränderung im Vergleich zum Ausgangszustand Ende 2006 (100\%)

\begin{tabular}{lccc}
\hline Baumart & \multicolumn{3}{c}{$\begin{array}{c}\text { C-Vorrat im Totholzspeicher } \\
{[\text { Mio. } \mathrm{C}]}\end{array}$} \\
& ertragsorientiert & naturnah & naturschutzorientiert \\
\hline Buche & 1,36 & 1,35 & 1,43 \\
& $(-56 \%)$ & $(-56 \%)$ & $(-54 \%)$ \\
Eiche & 2,88 & 2,85 & 2,75 \\
& $(-7 \%)$ & $(-8 \%)$ & $(-11 \%)$ \\
Fichte & 1,69 & 1,77 & 2,29 \\
& $(-22 \%)$ & $(-18 \%)$ & $(+6 \%)$ \\
Kiefer & 2,00 & 2,29 & 2,80 \\
& $(-8 \%)$ & $(+6 \%)$ & $(+29 \%)$ \\
\hline
\end{tabular}

\subsubsection{Produktpool}

Die Auswirkungen der waldbaulichen Szenarien setzen sich beim Produktpool und den einzelnen Produktklassen fort (s. Tab. 5). Dabei wurde für den Simulationszeitraum die heutige relative Verteilung des Laub- und Nadelholzes auf die einzelnen Produktklassen (s. Tab. 6) konstant gehalten, obwohl sie sich beispielsweise durch innovative Holzprodukte oder eine noch stärkere energetische Holznutzung künftig verändern kann.

Im Laufe der 30-jährigen Simulation wird der Produktspeicher durch die Holznutzungen immer weiter aufgefüllt. Die Unterschiede zwischen den Szenarien ergeben sich aus den jeweiligen Eingriffsstärken (s. Tab. 11). Am höchsten ist die Kohlenstoff-Akkumulation in Holzprodukten bei der ertragsorientierten Variante (60,2 Mio. t C), gefolgt von der naturnahen (52,4 Mio. t C) und der naturschutzorientierten Behandlung (36,2 Mio. t C). Entsprechend dem Holzverwendungsschlüssel wird bei allen drei waldbaulichen Szenarien der Laubholz-Produktpool am stärksten in der Produktklasse Energieholz erhöht, während es beim Nadelholz-Produktpool die Produktklasse der Produkte mit langer Lebensdauer ist (s. Abb. 23). 


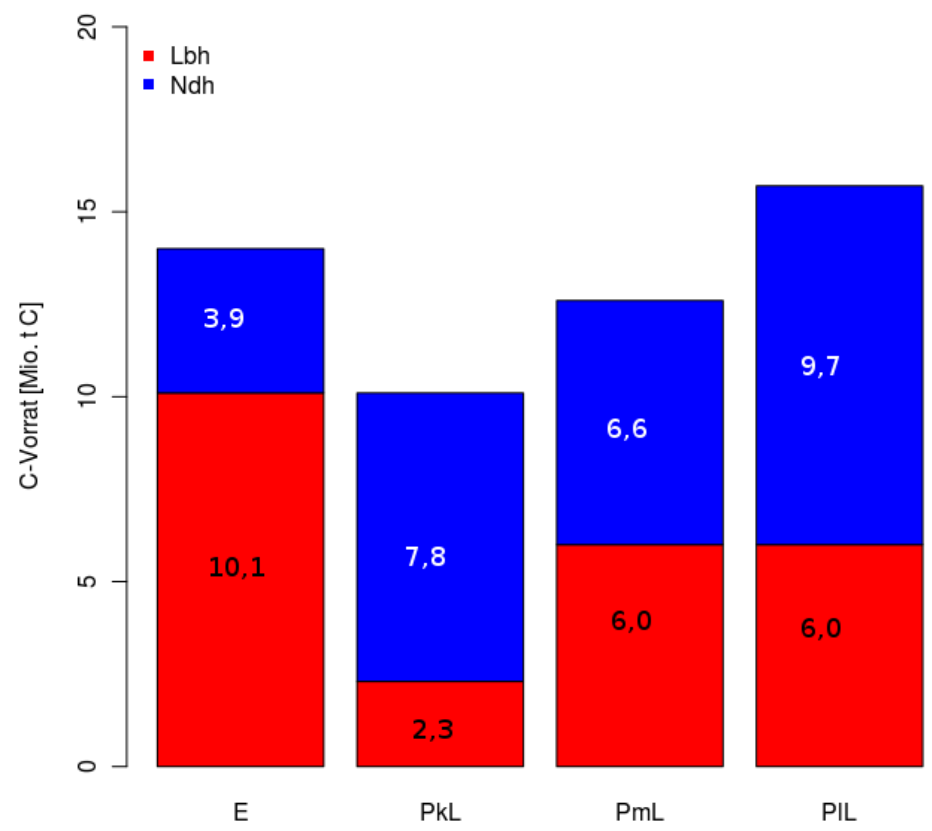

Abbildung 23: Akekumulierter C-Vorrat [Mio. tC] im Produktspeicher 2036 beim Szenario naturnaher $W$ aldbau getrennt nach Produktklassen $(E=$ Energiebolz, PkL, PmL, PlL = Produkte mit kurzer, mittlerer und langer Lebensdauer) sowie nach Laub- und Nadelboly.

Unter Beachtung der standörtlichen Zuordnungen, Flächenanteile und Altersklassenverhältnisse der Hauptbaumarten zu Beginn der Simulationen stellen sich die im Produktpool akkumulierten Volumina (s. Abb. 24a) und die darin gespeicherten C-Vorräte (s. Abb. 24b) für das Szenario naturnaher Waldbau an deren Ende wie folgt dar. Wegen der geringeren Dichten werden beim Nadelholz deutlich größere Volumina benötigt, um vergleichbare C-Vorräte wie beim Laubholz in den verschiedenen Produktklassen zu speichern. Die Produktklasse Energieholz wird beim Laubholz hauptsächlich durch das Buchenholz aufgebaut. Dies gilt abgeschwächt auch für die anderen Produktklassen. Lediglich bei den Produkten mit langer Lebensdauer erhöht sich der relative Anteil des Eichenholzes etwas. Beim Nadelholz spiegeln sich die höheren Fichtennutzungen auch in den gespeicherten C-Vorräten der einzelnen Produktklassen wider. Der Anteil der im Fichtenholz gespeicherten C-Vorräte in Produkten mit langer Lebensdauer ist absolut und relativ am höchsten. 
a)

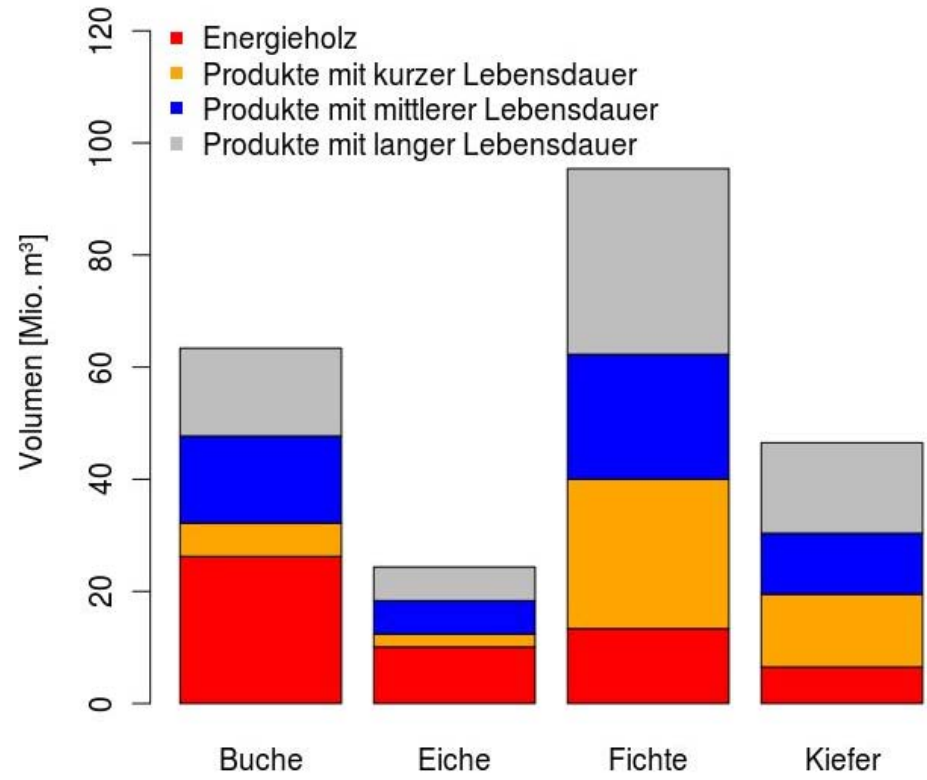

b)

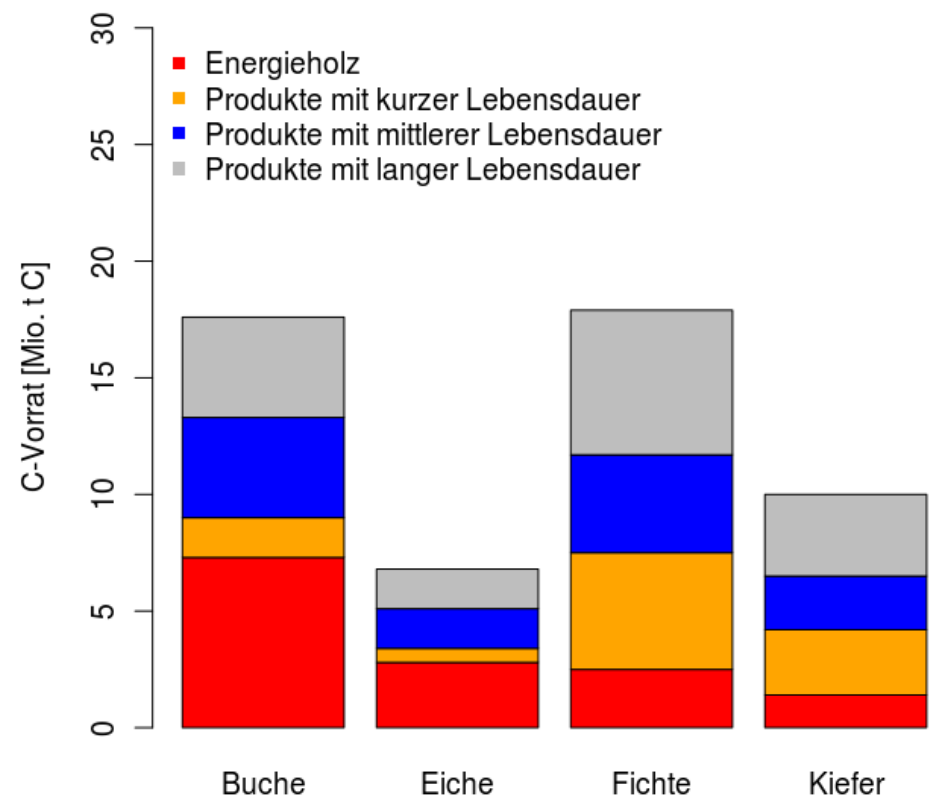

Abbildung 24: a) Akekumulierte Volumina [Mio. $\left.\mathrm{m}^{3}\right]$ und b) akkumulierte C-Vorräte [Mio. t C] im Produktpool für das Szenario naturnaher $W$ aldbau getrennt nach Produktklassen und Hauptbaumarten 
Im Vergleich zur naturnahen Behandlung erhöht sich beim ertragsorientierten Waldbau der C-Vorrat im Produktspeicher des Laubholzes um insgesamt $13 \%$ und bei den Produkten aus Nadelholz um $17 \%$. Demgegenüber nehmen die CVorräte des Laub- bzw. Nadelholzproduktspeichers bei der naturschutzorientierten Variante um $26 \%$ bzw. $35 \%$ ab (s. Tab. 13).

Tabelle 13: Relative Unterschiede [\%] im C-Vorrat der Holiprodukte aus Laub-bzw. Nadelholz. 2036 zwischen dem Szenario naturnaber Waldbau (100\%) und den Szenarien ertragsund naturschutzorientierter $W$ aldbau

\begin{tabular}{lcc} 
Holzgruppe & \multicolumn{2}{c}{ Relative Unterschiede im C-Vorrat der Holzprodukte } \\
& ertragsorientiert & naturschutzorientiert \\
\hline Laubholz & $113 \%$ & $74 \%$ \\
Nadelholz & $117 \%$ & $65 \%$ \\
\hline
\end{tabular}

Die energetische Substitution fossiler Brennstoffe durch die Produktklasse Energieholz ist beim Laubholz generell höher als beim Nadelholz. Durch das nach dem naturnahen Waldbauszenario anfallende Energieholz werden rund 70 Mio.t zusätzlicher $\mathrm{CO}_{2}$-Emissionen vermieden, die sonst bei der Verbrennung fossiler Brennstoffe in die Atmosphäre gelangen würden. Der Substitutionseffekt ist beim naturschutzorientierten Szenario mit rund 50 Mio. $\mathrm{CO}_{2}$ wesentlich geringer und beim ertragsorientierten Szenario mit 80 Mio. t $\mathrm{CO}_{2}$ noch merklich höher. 



\section{Diskussion}

Nachfolgend werden zunächst das Material und die Methoden der C-Studie diskutiert, danach die Veränderungen der Kohlenstoffspeicher zwischen der BWI ${ }^{1}$, der $\mathrm{BWI}^{2}$ und dem Ausgangszustand vor Einsetzen der Simulationsrechnungen Ende 2006 beleuchtet und anschließend die Entwicklungen der C-Speicher bei den drei unterschiedlichen waldbaulichen Szenarien besprochen.

\subsection{Material und Methoden}

Die vorliegende Studie ist nach der Arbeit von BösWALD u. WIERLING (1997) die zweite Untersuchung zum Thema Kohlenstoff im Wald im Auftrag des Niedersächsischen Ministeriums für Ernährung, Landwirtschaft, Verbraucherschutz und Landesentwicklung. Sie baut vor allem auf den Ergebnissen der Clusterstudie Forst und Holz Niedersachsen und der zweiten Bodenzustandserhebung (BZE II) auf. Die Ausgangsvorräte der Clusterstudie unterscheiden sich teilweise etwas von den Ergebnissen der $\mathrm{BWI}^{2}$. Dies erklärt sich dadurch, dass die Vorräte für die Clusteranalyse mit eigenen Funktionen auf der Basis der BWI ${ }^{2}$-Daten berechnet wurden.

Neben der lebenden Baumbiomasse werden als weitere C-Speicher auch die tote Baumbiomasse, die Bodenvegetation, der Mineralboden mit organischer Auflage und die aus niedersächsischem Holz erzeugten Holzprodukte in die Betrachtungen mit einbezogen. Damit wird für Niedersachsen ein weitgehend vollständiger Überblick über den Beitrag der Forst- und Holzwirtschaft zur Speicherung von Kohlenstoff im Ökosystem Wald und in den daraus hervorgegangenen Holzprodukten gegeben. Die Angaben zur C-Speicherung im Mineralboden basieren auf einer vorläufigen Auswertung ausgewählter BZE-Punkte. Außerdem ist die Erhebung der C-Vorräte im Boden sehr schwierig und kann mit vielen Fehlern behaftet sein (s. Kap. 2.3).

Zur Bestimmung der lebenden oberirdischen Baumbiomassen wurde nach einem intensiven Literaturstudium und einer Überprüfung verschiedener geeignet erscheinender Biomassefunktionen auf die baumartenspezifischen Funktionen von ZELL (2008) zurückgegriffen. Für die unterirdische Wurzelbiomasse wurden, teilweise wegen fehlender Alternativen, für alle vier untersuchten Hauptbaumarten die Biomassefunktionen von BOLTE et al. (2004) verwendet. Hierzu besteht offensichtlich noch Forschungsbedarf. Gleiches gilt auch für die Quantifizierung der CVorräte in der toten Baumbiomasse und der Bodenvegetation.

Weitgehendes Neuland wurde bei der Quantifizierung des additiven CSpeichers in Holzprodukten beschritten. Hierzu mussten auf der Grundlage eines intensiven Literaturstudiums Holzproduktklassen mit unterschiedlicher Lebens- 
dauer definiert und ein vereinfachter Holzverwendungsschlüssel festgelegt werden. Wegen fehlender Informationen waren hierzu einige Vergröberungen und Pauschalierungen unvermeidbar. Eine differenziertere Analyse der Holzströme würde wesentliche Verbesserungen bei der Zuordnung bestimmter Holzsortimente zu bestimmten Holzprodukten ermöglichen.

Die dreißigjährige Simulation der Entwicklung einzelner C-Speicher unter Zugrundelegung der drei verschiedenen Szenarien ertragsorientierter, naturnaher und naturschutzorientierter Waldbau ergab, dass die C-Speichermengen durch die Strategien der Forst- und Holzwirtschaft wesentlich beeinflusst werden können. Bezogen auf das Bestandesleben unserer Hauptbaumarten war aber der Simulationszeitraum zu kurz, weil die Auswirkungen der waldbaulichen Strategien noch zu stark von den gegenwärtigen Baumartenanteilen, ihrer standörtlichen Zuordnung und ihrem Altersaufbau beeinflusst werden. Bei den Nutzungen konnten die Effekte einer Kaskadennutzung nur angesprochen, aber nicht berücksichtigt werden, weil hierzu entsprechende Datengrundlagen fehlten.

\subsection{C-Speicher 2002 und Ende 2006}

\subsubsection{Lebende Baumbiomasse}

Der Vergleich der Gesamtderbholzvorräte zu den Stichtagen der BWI 1987 und der BWI 2002 und der darin gespeicherten C-Vorräte in Höhe von ca. 50 Mio. t C bzw. ca. 76 Mio. t C dokumentiert die in den letzten Jahren stark gestiegene Kohlenstoff-Senkenleistung der niedersächsischen Wälder (s. Kap. 4.1). Der Vorratsanstieg erklärt sich vor allem aus dem unausgeglichenen Altersklassenaufbau der Bestände und aus Hiebssätzen, die deutlich unter dem Zuwachs lagen. Bis zum Ende des Jahres 2006 kam es gegenüber 2002 zu einer leichten Absenkung der Derbholz- und C-Vorräte, weil auch die Schäden des Orkans Kyrill mit Blick auf die Startwerte der Simulationsrechnungen bereits berücksichtigt wurden. Die CVorräte in den Kiefernbeständen waren höher als diejenigen in den Buchen-, Eichen- und Fichtenbeständen (s. Tab. 7).

Der gesamte C-Vorrat des Derbholzvorrates betrug Ende 2006 rund 72 Mio. t C bzw. im Mittel 66 t C/ha begehbarer Holzbodenfläche. Im Vergleich zu den deutschlandweiten Werten von DUNGER et al. (2009) sowie DiETER und ELSASSER (2002) sind die C-Vorräte in den niedersächsischen Wäldern geringer. Die Differenzen lassen sich zum einen durch die schwächeren Standorte im niedersächsischen Tiefland und den hohen Flächenanteil jüngerer Fichten- und Kiefernbestände erklären (s. Abb. 4). Zum anderen ist das Ertragsniveau in den süddeutschen Bundesländern wesentlich höher als in Niedersachsen, was sich entsprechend auf die Vorräte und Zuwächse der einzelnen Baumarten auswirkt.

Für die Buche ist der für das Jahr 2006 berechnete C-Vorrat in Höhe von durchschnittlich $133 \mathrm{t} \mathrm{C/ha} \mathrm{ähnlich} \mathrm{hoch} \mathrm{wie} \mathrm{derjenige} \mathrm{in} \mathrm{Thüringen} \mathrm{(WIRTH} \mathrm{et} \mathrm{al.}$ 
2004a), wenn man den Flächenanteil der Buche nach den Ergebnissen der BWI² zu Hilfe nimmt. Etwas geringer sind die C-Vorräte der Buchenbestände in Nordrhein-Westfalen (JOOSTEN et al. 2004). In Baden-Württemberg (PISTORIUS 2007) und in der Hainich-Dün-Region (MUND 2004) wurden größere C-Vorräte in bewirtschafteten Buchenbeständen festgestellt. Dies muss im Zusammenhang mit den Standorten, den Ertragsniveaus und dem Altersaufbau gesehen werden. Die Literaturstudie von JACOBSEN et al. (2003) weist deutschlandweit höhere C-Vorräte für Buchenbestände aus, als sie für Niedersachsen berechnet wurden.

Der durchschnittliche C-Vorrat der Eichenbestände im Jahr 2006 in Höhe von $96,4 \mathrm{tC} / \mathrm{ha}$ ist im Vergleich zu Untersuchungen in Baden-Württemberg und Bayern als gering anzusehen. In Bayern waren die Kohlenstoffvorräte um knapp $16 \mathrm{t} /$ ha höher (BÖSWALD 1996), in Baden-Württemberg um etwa $6 \mathrm{t} / \mathrm{ha}$ (PISTORIUS 2007). Demgegenüber lagen die entsprechenden C-Vorräte in Thüringen knapp unter den niedersächsischen Werten (WIRTH et al. 2004a).

Für alle Baumarten gilt, dass die Vergleiche mit den C-Vorräten in anderen Bundesländern nur eine Orientierung geben können, da deren Höhen vielfältigen Einflussgrößen unterliegt.

Dies zeigt sich ebenfalls bei den entsprechenden süddeutschen Untersuchungen zur Fichte (s. Kap. 3.1.1). Aus den Biomassen der analysierten bayerischen Fichtenbestände sind sehr hohe C-Vorräte ableitbar. Diese gelten aber nur für die jeweiligen Bestände und sind weder auf Regionen noch auf das Bundesland übertragbar. In Niedersachsen lag 2006 der C-Vorrat in Fichtenbeständen bei durchschnittlich 86,7 t C/ha. Für Bayern gibt BÖSWALD (1996) für die fichtendominierte Baumartengruppe Fichte-Tanne-Douglasie einen C-Vorrat von $120 \mathrm{tC} / \mathrm{ha}$ an. Noch höher, aber auch später ermittelt und zugleich Ausdruck eines Fichten-Altholzüberhanges, ist nach den Ergebnissen der $\mathrm{BWI}^{2}$ der C-Vorrat für Fichte in Baden-Württemberg mit 132,6 t C/ha (PISTORIUS 2007).

Für die Kiefer ergibt sich ein ähnliches Bild wie bei der Fichte. Der geschätzte mittlere C-Vorrat liegt 2006 bei 67,7 t C/ha (s. Tab. 7). Für Bayern (BÖSWALD 1996) und Baden-Württemberg (PISTORIUS 2007) werden um rund $30 \mathrm{t} C / \mathrm{ha}$ höhere Werte angegeben, während in Thüringen die C-Vorräte in den Kiefernbeständen mit durchschnittlich $61 \mathrm{t} \mathrm{C/ha} \mathrm{vergleichbar} \mathrm{sind} \mathrm{(WIRTH} \mathrm{et} \mathrm{al.} \mathrm{2004a).}$ Sehr viel geringer ist der aus den Ergebnissen von MUCHIN et al. (2008) ableitbare C-Vorrat für Kiefer in Brandenburg in Höhe von 38 t C/ha (s. Kap. 3.1.1).

Die in den Arbeiten von WirTH et al. (2004a) und PISTORIUS (2007) festgestellte Reihung der Hauptbaumarten nach ihren mittleren C-Vorräten $(\mathrm{Bu}>\mathrm{Ei}$, $\mathrm{Fi}>\mathrm{Ki})$ kann durch die Ergebnisse der vorliegenden Untersuchung für Niedersachsen weitgehend bestätigt werden $(\mathrm{Bu}>\mathrm{Ei}>\mathrm{Fi}>\mathrm{Ki})$. Dies wird sich zukünftig sicherlich ändern, wenn sich der hohe Flächenanteil der jüngeren Fichten- und Kiefernbestände der zweiten und dritten Altersklasse in höhere Altersklassen verschiebt und sich deren Anteil an der Kohlenstoffspeicherung erhöht. Parallel dazu wird der bis dato große C-Vorrat der Buchen in den Altersklassen VI und 
älter (Alter $>100$ Jahre) bald zur Nutzung anstehen und dem Produktpool zugeführt werden.

\subsubsection{Tote Baumbiomasse}

Das Totholzvolumen 2006 ist wesentlich höher als zum Stichtag der BWI ${ }^{2}$ (BMELV 2005) (s. Kap. 4.2.2). Im Vergleich der Laub- und Nadelbaumarten weisen Buche und Eiche mit 3,1 Mio. t $\mathrm{C}$ deutlich höhere C-Vorräte im Totholz auf als Fichte und Kiefer mit insgesamt 2,17 Mio. t C. Das flächengewogene Mittel der Hauptbaumarten in Niedersachsen ergibt einen C-Vorrat im Totholzpool von rund $3 \mathrm{t} \mathrm{C} / \mathrm{ha}$ zu Beginn der Simulation. Im Vergleich zu den von PREGITZER und EUSKIRCHEN (2004) angegebenen mittleren C-Gesamtvorräten im Totholz für Wälder der gemäßigten Zone in Höhe von $42 \mathrm{t} \mathrm{C/ha} \mathrm{sind} \mathrm{die} \mathrm{Werte} \mathrm{dieser} \mathrm{Studie}$ als sehr gering anzusehen. Der durch Fortschreibung errechnete C-Vorrat von rund 2,5 Mio. t C bzw. 2,2 t C/ha für alle Baumarten entspricht aber in etwa den Angaben von WIRTH et al. (2004a) für Wirtschaftswälder in Mitteleuropa.

Die Totholzzersetzung ist ein wichtiger Aspekt für die C-Speicherung im Totholz. Sie wird in dieser Studie durch die baumartenspezifischen linearen Funktionen von MEYER et al. (2009) quantifiziert. Dabei kommt es zu Unschärfen, weil diese Zersetzungsfunktionen ab einem Durchmesser von $20 \mathrm{~cm}$ gültig sind, während in dieser Studie das Totholz ab der Grenze von $7 \mathrm{~cm}$ Durchmesser betrachtet wurde (s. Kap. 1.3). MÜLLER-USING und BARTSCH (2009) fanden für Buchentotholz mit einem Durchmesser größer als $10 \mathrm{~cm}$ heraus, dass es unabhängig vom Durchmesser nach 35 Jahren zersetzt ist. Die von MEYER et al. (2009) ermittelte Zersetzungsdauer beträgt für Buche 43 Jahre, für Eiche 78 Jahre, für Fichte 55 Jahre und für Kiefer 50 Jahre. Da diese Abbauzeiträume teilweise weit über den Simulationszeitraum hinausgehen, erscheinen die Funktionen von MEYER et al. (2009) dennoch für diese Studie als geeignet. Zudem wird der Abbau von Totholz von sehr vielen weiteren Faktoren beeinflusst, wie z. B. dem Klima (vgl. MACKENSEN et al. 2003, MEYER et al. 2009), die in dieser Untersuchung nicht berücksichtigt werden konnten.

\subsubsection{Mineralboden und Bodenvegetation}

Für die Analyse des Bodenpools war die getrennte Betrachtung von Humusauflage und Mineralböden einerseits und Mooren sowie anmoorigen Böden andererseits wichtig, da Moore und anmoorige Böden in einem weitaus höheren Maße C speichern (s. Kap. 4.2.5). Ihr Anteil beträgt zwar nur ca. $1 \%$ an der niedersächsischen Waldfläche, doch ist ihr C-Vorrat pro Hektar fast viermal so hoch wie derjenige in mineralischen Böden. Die Erhaltung der Moore und der anmoorigen Böden ist daher sehr wichtig (NABUURS et al. 2008) und ein wesentlicher Bestandteil einer nachhaltigen, multifunktionalen Forstwirtschaft (SPELLMANN et al. 2004). 
Zudem leisten diese Standorte auch einen wichtigen Beitrag zum Erhalt der biologischen Vielfalt (MEYER et al. 2009).

In den Böden Niedersachsens bestehen große Unterschiede in den jeweiligen C-Vorräten. Der Einfluss der Bestockung auf diese Vorräte ist im Zusammenhang mit dem bevorzugten Anbau der Laubbaumarten auf den eher besseren und der Nadelbaumarten auf den eher schlechteren Standorten zu sehen. So wurde unter Laubbaumbeständen ein C-Vorrat im Mineralboden von ca. 72 t C/ha und unter Nadelbaumbestände von ca. 103 t C/ha ermittelt.

Die unterschiedlichen C-Vorräte in der Humusauflage von Laub- und Nadelwaldbeständen bzw. zwischen den entsprechenden Werten im Bergland und im Tiefland sind ebenfalls im Zusammenhang mit mehreren Standortsfaktoren zu sehen, die aber nicht in die Analysen mit einbezogen werden konnten. Zu diesen Standortsfaktoren zählen das Klima, die Wasser- und Nährstoffversorgung, die Artenzusammensetzung der Bodenfaumna, die Bodentextur und die Topographie. Entscheidende anthropogene Faktoren sind die aktuelle Bestockung, die waldbauliche Behandlung sowie die historische und aktuelle Landnutzung (WIRTH et al. 2004a). Es wird davon ausgegangen, dass die langfristige Speicherung von $\mathrm{C}$ im Bodenpool von sehr langsamen Abbauraten und Umsatzzeiten von einem Jahrhundert bis zu mehreren Jahrtausenden bestimmt wird (LORENZ u. LAL 2010).

In jedem Fall ist der Waldboden ein wichtiger C-Speicher, welcher mindestens ebenso viel, im Fall der analysierten Böden unter Nadelbaumbeständen sogar doppelt soviel $\mathrm{C}$ speichert wie die oberirdische lebende Baumbiomasse. Bei forstwirtschaftlichen Maßnahmen sollten daher nur bodenschonende Verfahren zum Einsatz kommen, die diesen wichtigen C-Speicher nicht gefährden.

Der Biomasse und dem C-Vorrat der Bodenvegetation konnte im Rahmen dieser Studie nicht direkt nachgegangen werden, weil eine flächendeckende Datengrundlage fehlte. Es wurde daher auf Ergebnisse aus Thüringen zurückgegriffen (s. Kap. 3.3). Hier zeigten sich sehr deutliche Unterschiede zwischen den Lichtbaumarten Eiche und Kiefer einerseits sowie den anderen untersuchten Baumarten andererseits. Angesichts des geringen C-Vorrates der Bodenvegetation (BURSCHEL et al. 1993) im Vergleich zur lebenden Baumbiomasse (s. Kap. 3.3 u. Tab. 7) wurde dieser Speicher vereinfachend als konstant betrachtet (vgl. PISTORIUS 2007). SCHULZE et al. (2002) geben aber an, dass die C-Bindung in der oberirdischen Bodenvegetation in Mitteleuropa etwa 10 bis $20 \%$ derjenigen der Baumschicht erreichen kann.

\subsection{Szenarienvergleich}

Die waldbaulichen Szenarien bilden drei Bewirtschaftungskonzepte ab, die ungeachtet der Eigentumsform so oder so ähnlich in deutschen Forstbetrieben praktiziert werden. Abweichend von der betrieblichen Wirklichkeit, für die die Vielfalt des waldbaulichen Handels der mehreren Tausend Waldbesitzer kennzeichnend ist, 
wurden in den Simulationsläufen der für diese Studie grundlegenden Clusterstudie Forst und Holz Niedersachsen die Konzepte mit ihren naturschutzorientierten und eingriffsspezifischen Regeln vollflächig auf der Basis der fortgeschriebenen $\mathrm{BWI}^{2}-$ Modellbestände umgesetzt (s. Tab. 1). Hierdurch können die Auswirkungen der Szenarien auf die Höhe der C-Vorräte in der lebenden und toten Baumbiomasse sowie im Produktpool in ihrer reinen Form besser erfasst und beurteilt werden. Die C-Vorräte im Boden (Mineralboden + Humusauflage) und in der Bodenvegetation wurden als konstant angesehen.

Die zwischen den Szenarien naturnaher, ertragsorientierter und naturschutzorientierter Waldbau variierenden Naturschutzstandards, Eingriffsintensitäten und Zielstärken (Produktionszeiträume) erfüllen ihre Wirkung und führen zu etwa gleichbleibenden, geringeren bzw. höheren Derbholz- und C-Vorräten in der lebenden und toten Baumbiomasse gegenüber den Ausgangswerten Ende 2006. Die genutzten Holzmengen und die in ihnen gespeicherten C-Vorräte reichen in dem 30-jährigen Simulationszeitraum von 295 Mio. $\mathrm{m}^{3}$ bzw. 74 Mio. t $\mathrm{C}$ beim naturnahen Waldbau, über 352 Mio. $\mathrm{m}^{3}$ bzw. 88 Mio. t $\mathrm{C}$ beim ertragsorientierten Waldbau bis hin zu nur 201 Mio. $\mathrm{m}^{3}$ bzw. 50 Mio. t C beim naturschutzorientierten Waldbau. Nicht enthalten sind in diesen Zahlen die additiven Effekte durch die unterschiedlichen Verweildauern der aus dem Rohholz hergestellten Holzprodukte.

Die jeweils simulierten Waldbaukonzepte beeinflussen auch den mittleren jährlichen Volumenzuwachs und die darin gespeicherten C-Vorräte. Diese sind beim naturschutzorientierten Szenario etwas höher als beim naturnahen und ertragsorientierten (s. Tab. 14). Da beim ertragsorientierten Szenario mehr genutzt wird als nachwächst, werden bei dieser Variante in dem 30-jährigen Simulationszeitraum die Derbholz- und C-Vorräte dementsprechend abgesenkt und mehr Masse dem Produktpool zugeführt. Beim naturschutzorientierten Szenario verhält es sich umgekehrt und beim naturnahen Szenario werden die Niveaus in etwa gehalten.

Tabelle 14: Übersicht über den mittleren jährlichen Volumenquwachs $\left[\mathrm{m}^{3} / \mathrm{ha}^{*} \mathrm{a}\right]$ und den darin gespeicherten $C-V$ orrat $\left[t C / h a^{*} a\right]$ der verschiedenen Szenarien

\begin{tabular}{lcc}
\hline Szenario & $\begin{array}{c}\text { Volumenzuwachs } \\
{\left[\mathrm{m}^{3} / \mathrm{ha}^{*} \mathrm{a}\right]}\end{array}$ & $\begin{array}{c}\mathrm{C}-\text { Vorrat } \\
{[\mathrm{t} \mathrm{C} / \mathrm{ha} \text { a }]}\end{array}$ \\
\hline ertragsorientiert & 9,6 & 2,4 \\
naturnah & 10 & 2,5 \\
naturschutzorientiert & 10,5 & 2,6 \\
\hline
\end{tabular}

Bezogen auf die Hauptbaumarten wird am Ende des Simulationszeitraumes und unabhängig von dem betrachteten waldbaulichen Szenario der höchste C-Vorrat in 
der lebenden Biomasse der Kiefernwälder gespeichert. Er liegt zwischen 14,47 Mio. t C beim ertragsorientierten und 25,43 Mio. t C beim naturschutzorientierten Szenario. Danach folgen Buche und Fichte und schließlich die Eiche (s. Tab. 10). Diese Unterschiede sind im Zusammenhang mit den Flächenanteilen der Baumarten, ihrem Altersklassenaufbau und den unterschiedlichen Nutzungsmengen zu sehen.

Auch der Totholzspeicher wird durch die untersuchten Szenarien unterschiedlich stark beeinflusst (s. Tab. 12). Da sich der Totholzpool hauptsächlich aus den baumartenspezifischen Ernteresten speist, ist die relative Änderung der C-Vorräte zwischen 2007 und 2036 beim ertragsorientierten Szenario für die Baumarten Buche und Fichte am höchsten (s. Abb. 19 bis 22). Bei der Kiefer gibt es zum Ende der Simulation eindeutige Unterschiede zwischen den Szenarien. Durch die naturnahe und ertragsorientierte Behandlung wird annähernd der Ausgangswert von Ende 2006 erreicht und durch das naturschutzorientierte Szenario wird der CVorrat erhöht. Dieser Unterschied erklärt sich vor allem aus dem Altersaufbau der niedersächsischen Kiefernbestände. Damit gibt es keinen eindeutigen Trend für alle untersuchten Baumarten, jedoch lässt sich festhalten, dass das Szenario naturnaher Waldbau jeweils eine Mittelstellung einnimmt. Insgesamt wird der von PREGITZER und EUSKIRCHEN (2004) für Wälder der gemäßigten Zone genannte, bemerkenswert hohe C-Vorrat im Totholz von $42 \mathrm{t} \mathrm{C/ha} \mathrm{nicht} \mathrm{erreicht.} \mathrm{Dies} \mathrm{ist}$ unter anderem auf die Aufnahmegebiete (gemäßigte Zonen in Nord- und Südamerika) zur Berechnung dieses C-Vorrates im Totholz zurückzuführen.

Der Produktpool wird bestimmt durch die zur Verfügung stehenden Rohholzmengen und Sortimente, die jeweilige Holzverwendung und die Lebensdauer der einzelnen Holzprodukte. Mittels eines vereinfachten Holzverwendungsschlüssels wurden verschiedene Sortimente unterschiedlichen Produkten und Produktklassen zugeordnet (s. Kap. 3.5). Die Ergebnisse sind daher mit Unsicherheiten behaftet.

Aus der Entwicklung der Produktspeicher ist ersichtlich, dass sie sowohl beim Laub- als auch beim Nadelholz am stärksten durch die höheren Nutzungen des ertragsorientierten Szenarios aufgefüllt werden, während die naturschutzorientierte Variante erwartungsgemäß die geringsten Zuwachsraten aufweist. Laubholz, und hier insbesondere das Buchenholz, wird überwiegend als Energieholz verwandt. Die relativ geringe Bedeutung des Laubholzes für die Papierherstellung spiegelt sich in dem geringen Anteil der Produkte mit kurzer Lebensdauer wider. Bemerkenswert sind die relativ hohen Anteile bei den Produkten mit langer (Bauholz, Fußböden) und mittlerer Lebensdauer (Holzwerkstoffe, Möbel, Furniere). Sie unterstellen eine entsprechende Laubholznachfrage an den Märkten, die aber im letzten Jahrzehnt nicht gegeben war (ZMP 2008). Im Gegenteil, der Laubschnittholzmarkt stagniert auf einem niedrigen Niveau, der Furnier- und Möbelholzmarkt ist rückläufig und die Holzpreise für die Buche sind für die Waldbesitzer unbefriedigend. Umgekehrt sehen die Marktverhältnisse beim Nadelholz aus, bei dem sich bereits Versorgungsengpässe abzeichnen (SPELLMANN 2010). Aus Nadelholz 
werden vorwiegend Produkte mit langer Lebensdauer generiert. Der größte Teil wird im Hausbau verwendet, wo der Kohlenstoff langfristig sequestriert wird. Das Fichten- und Kiefernindustrieholz ist die wichtigste Rohstoffgrundlage für die Papier- und Holzwerkstoffindustrie, deren Produkte eine kurze bzw. mittlere Lebensdauer haben. Deren Pool wird z.T. auch von den 39\% Sägerestholz gespeist, die beim Einschnitt von Nadelstammholz anfallen (s. Kap. 3.5.3). Größere Anteile des Sägerestholzes werden aber auch in den Werken zur eigenen Energieerzeugung genutzt, was den Anteil Energieholz bei Fichte und Kiefer erklärt (s. Abb. 24a und b). Die Bedeutung des Roh- und Altholzes für die energetische Substitution fossiler Energieträger (C-Vermeidung) darf nicht unterschätzt werden. Der Anteil der Biomasse an der Primärenergiebereitstellung aus erneuerbaren Energien betrug im Jahre 2009 fast $70 \%$ (Windkraft 12\%, Wasserkraft 6\%, Photovoltaik $2 \%$ ). Von diesen $70 \%$ entfielen mehr als $50 \%$ auf Energieholz (AG ENERGIEBILANZEN 2010).

Um zu einer Gesamtbeurteilung der Szenarien aus Sicht der Kohlenstoffspeicherung zu kommen, ist es wichtig, die Grenzen des zu prüfenden Systems genau zu definieren. Im Kontext der C-Sequestrierung im Wald gibt es zwei Definitionsmöglichkeiten: Entweder wird der sogenannte Waldökosystemansatz gewählt, d. h. das Waldökosystem wird nur innerhalb seiner Systemgrenzen betrachtet, oder es wird ein umfassenderer Ansatz gewählt, der neben dem Wald auch den Forst- und Holzsektor mit einschließt (s. Kap. 1.1).

Im Rahmen des Kyoto-Protokolls und insbesondere des Artikel 3.4 ist es möglich, die Waldbewirtschaftung in die nationale Treibhausgasbilanz mit einzuberechnen. Davon macht Deutschland seit 2006 Gebrauch, ohne dass bisher eine Anrechnung der Holzprodukte auf die C-Speicherung möglich ist (HEUER 2010). Der Waldökosystemansatz führt heute dazu, dass Holznutzungen C-Freisetzungen gleichgesetzt werden (KRUG 2010, PROFFT 2010). Somit werden nur Vorratsanhebungen im Wald positiv bewertet.

Bislang konnte Deutschland von diesem Ansatz profitieren, da in den letzten Jahrzehnten die Derbholzvorräte in den Wäldern kontinuierlich angestiegen sind (BMELV 2009). Mittlerweile ist aber mit einem Gesamtderbholzvorrat in Höhe von ca. 3,6 Mrd. $\mathrm{m}^{3}$ ein Niveau erreicht (Rang 1 in Europa), das in nächster Zeit zwangsläufig zu höheren Nutzungen (Altersaufbau) oder zu größeren Kalamitäten (Sturmrisiko bei Fichte) führen und den deutschen Wald zur „ $\mathrm{CO}_{2}$-Quelle“ machen wird. Ändert sich nicht bald der Bewertungsansatz, so würde damit ein Land bestraft, dass für seine nachhaltige, vorratspflegliche Forstwirtschaft international anerkannt ist. Dies würde auch Niedersachsen treffen, dessen Wald aufgrund der Reparationshiebe, großflächigen Erstaufforstungen und den Schäden durch den Jahrhundertsturm vom 13.11.1972 nach wie vor ein Aufbaubetrieb ist.

Bei den Klimaverhandlungen der UN 2009 in Kopenhagen konnten sich die Staaten weder auf verbindliche, nachprüfbare Ziele der Reduzierung der Treibhausgase einigen noch wurden neue Anrechnungsregeln für die Waldbewirtschaf- 
tung verbindlich festgelegt. Es konnte nur ein Kompromiss ausgehandelt werden, in welchem die Referenz-Methode ${ }^{6}$, die Anrechnung von Holzprodukten und der Umgang mit natürlichen Störungen enthalten ist. Zusätzlich ist die anrechenbare $\mathrm{CO}_{2}$-Menge der Waldbewirtschaftung einer auch jetzt schon bestehenden Kappungsgrenze unterworfen. Die Verhandlungen über diesen Mittelweg sollten bei der Vertragsstaatenkonferenz des Kyoto-Protokolls in Mexiko Ende 2010 abgeschlossen werden (HEUER 2010). Dies war aber leider nicht der Fall. Die Verhandlungen werden in nächster Zeit weitergeführt, wobei im Verhandlungstext weiterhin die Holzprodukte als Pool der Waldbewirtschaftung aufgeführt sind (AWGKP 2010).

Nach dem bisher gültigen Waldökosystemansatz führen die drei waldbaulichen Szenarien bis 2036 bei den vier Hauptbaumarten zu deutlichen Unterschieden in den akkumulierten C-Vorräten in der lebenden Baumbiomasse und im Totholz. Die Differenz zwischen dem ertragsorientierten und dem naturschutzorientierten Szenario beträgt 39,5 Mio. t C. Werden die Holzprodukte einbezogen, verringert sich der Unterschied auf 15,4 Mio. t C. Dies unterstreicht die Notwendigkeit, die Holzprodukte in die Kohlenstoffbilanzen einzubeziehen (s. Abb. 25).
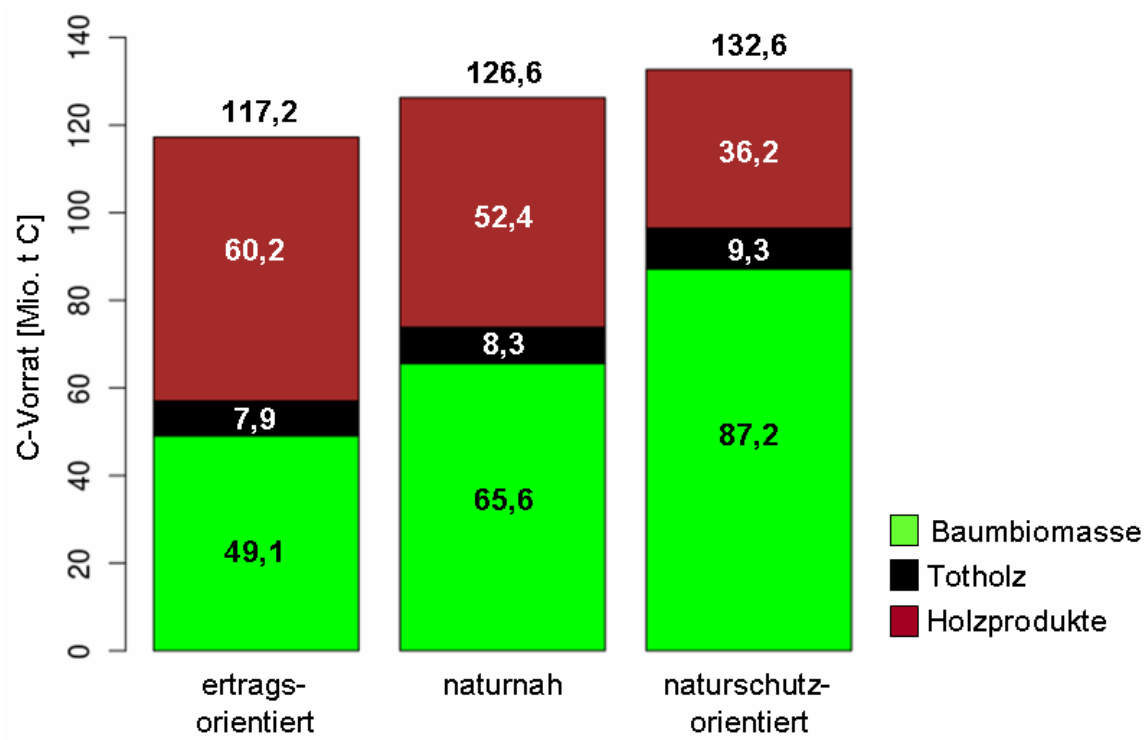

Abbildung 25: Ake.umulierter C-Vorrat [Mio. t C] in der lebenden Baumbiomasse, im Totholz und in den Holzprodukten der vier Hauptbaumarten im Jahr 2036 nach Behandlungsszenarien

6 Die Netto-Emissionen der Verpflichtungsperiode werden einem vorher vereinbartem Referenzwert gegenübergestellt. Der Refenzwert entspricht den Netto-Emissionen des in die Zukunft projektierten „,business as usual“ (HEUER 2010). 
In den Summenwerten sind die C-Speicher Boden und Bodenvegetation nicht enthalten, die die geringen Unterschiede überlagern würden. Die berechnete höhere C-Speicherung bei der naturschutzorientierten Variante ergibt sich aus den höheren C-Vorräten in der lebenden Baumbiomasse und im Totholzpool bei relativ geringen Nutzungsmengen. Das naturnahe Szenario verringert den C-Vorrat in der lebenden Baumbiomasse moderat und hebt die C-Speicherung gegenüber dem derzeitigen Stand deutlich an. Gleichzeitig werden die Nutzungen von durchschnittlich 4,3 Efm. o. R. zwischen BWI ${ }^{1}$ und BWI ${ }^{2}$ bis zum Jahr 2036 deutlich auf 7,2 Efm. o. R. angehoben und mit den darin gespeicherten C-Vorräten dem Produktpool zugeführt. Das ertragsorientierte Szenario verringert den Derbholzvorrat am stärksten und damit auch den C-Vorrat im Pool der lebenden Baumbiomasse. Der C-Vorrat im Totholz ist im Vergleich zur $\mathrm{BWI}^{2}$ gesunken und es werden den Wäldern die höchsten Nutzungsmassen entzogen (s. Tab. 10, 11 u. 12).

Auf Bundesebene haben sich KÖHL et al. (2009) intensiv mit der C-Speicherung in Wäldern in Abhängigkeit von verschiedenen Behandlungskonzepten beschäftigt. Dabei reichen die angewandten waldbaulichen Szenarien von einem generellen Nutzungsverzicht bis zu einer Maximierung der Gewinne aus der Waldbewirtschaftung mit einer starken Ausrichtung auf die Fichte. Sie kommen zu dem Ergebnis, dass die den Gewinn maximierende Variante im Zeitraum von 30 Jahren zu einer Speicherleistung im Bestand von $110 \mathrm{tC} / \mathrm{ha}$ führt und einen energetischen Substitutionseffekt von $84 \mathrm{tC} /$ ha bewirkt, was einem Gesamteffekt in Höhe von $194 \mathrm{t} C /$ ha entspricht. Im Gegensatz dazu führt die Nullnutzung zu einer Erhöhung des C-Speichers im Bestand auf 245 t C/ha. Somit schneidet diese Variante aus dem Blickwinkel der Kohlenstoffspeicherung besser ab, jedoch nach Ansicht der Autoren nur solange, wie sich die ungenutzten Bestände in der Phase des Vorratsaufbaus befinden. Weiterhin gehen sie davon aus, dass sich in ungenutzten Wäldern nach dem Erreichen der Gleichgewichtsphase eine negative Kohlenstoffbilanz im Vergleich zu bewirtschafteten Wäldern einstellt. Diese These, dass alte Wälder kaum noch $\mathrm{C}$ binden, wird durch andere Arbeiten angezweifelt (vgl. HESSENMÖLLER et al. 2008, LUYSSAERT et al. 2008). Dabei wird die Annahme getroffen, dass in ungenutzten Wäldern ein kleinflächiges Mosaik verschiedener Altersstufen entsteht, bei dem die jüngeren Bäume die Verluste in der C-Speicherung durch das Absterben der alten Bäume ausgleichen. Beispielsweise wird in der Arbeit von HeSSENMÖLLER et al. (2008) die These des großflächigen altersbedingten Zusammenbruchs des untersuchten Buchenwaldes im Nationalpark Hainich skeptisch gesehen. Hierzu ist aber zum einen anzumerken, dass nicht Buchenwälder, sondern Nadelwälder mit anderen biotischen und abiotischen Risiken in Deutschland wie auch in Niedersachsen überwiegen. Zum anderen sind Buchenwälder im Hainich, wie die meisten Buchenwälder in Deutschland, mehr oder weniger gleichaltrig und weisen ganz andere Strukturen auf als die noch vorhandenen Buchen-Urwälder in Ost- und Südosteuropa (vgl. KORPEL 1995, TABAKU u. MEYer 1999, MEYer et al. 2003). Dies zeigen auch die Uraltbuchen im Naturwald „Heilige Hallen“ in Mecklenburg-Vorpommern, die sich am Ende

Beiträge aus der NW-FVA, Band 6, 2011 
der Alterungsphase befinden und fortschreitend flächig zusammenbrechen. Auch HASENAUER (2011) kommt nach dem Vergleich von Simulationsrechnungen für Fichte und Buche im österreichischen Urwald Rothwald und in einem bewirtschafteten Fichtenbestand zum dem Schluss, dass bewirtschafteter Wald vor dem Erreichen der physiologischen Altersgrenze und dem Eintritt der Zerfallsphase genutzt wird und eine Kohlenstoffsenke darstellt, während der Naturwald mit dem Eintritt in die Zerfallsphase zur Kohlenstoffquelle wird.

Hinter dieser Diskussion steht letztendlich die Frage, welche Funktionen unsere Wälder haben und ob diese durch den bisher in Deutschland verfolgten integrativen Ansatz einer nachhaltigen, multifunktionalen Forstwirtschaft erfüllt werden sollen oder eher durch einen segregativen Ansatz mit großen Schutzgebieten und Plantagen bzw. sehr intensiv genutzten Wäldern wie in Nordeuropa, Südafrika oder Nordamerika. Vieles spricht in unserer dicht besiedelten Kulturlandschaft für ein Festhalten an der nachhaltigen, multifunktionalen Forstwirtschaft, die versucht, auf dem Wege des Kompromisses die vielfältigen Ansprüche an den Wald, einschließlich der Kohlenstoffspeicherung, angemessen zu berücksichtigen.

Die Entwicklungen bei den Verhandlungen zum Nachfolgevertrag des KyotoProtokolls, legen es jedenfalls nahe, die Holzprodukte als Teil des Forst- und Holzsektors in die Bewertung waldbaulicher Strategien mit einzubeziehen. Aber auch unabhängig von der zukünftigen Entwicklung der Klimapolitik ist es notwendig, die Maßnahmen zur Senkung der Treibhausgasemissionen und zur Erhöhung der Kohlenstoffspeicherung besser aufeinander abzustimmen (vgl. PROFFT 2010). So sollte die C-Senkenwirkung von Wäldern nicht länger isoliert von den Speicherleistungen und Substitutionseffekten der Holzprodukte gesehen werden. Dies öffnet den Blick von der Urproduktion über den industriellen Sektor bis hin zum nachgelagerten Bereich (s. Abb. 26). 


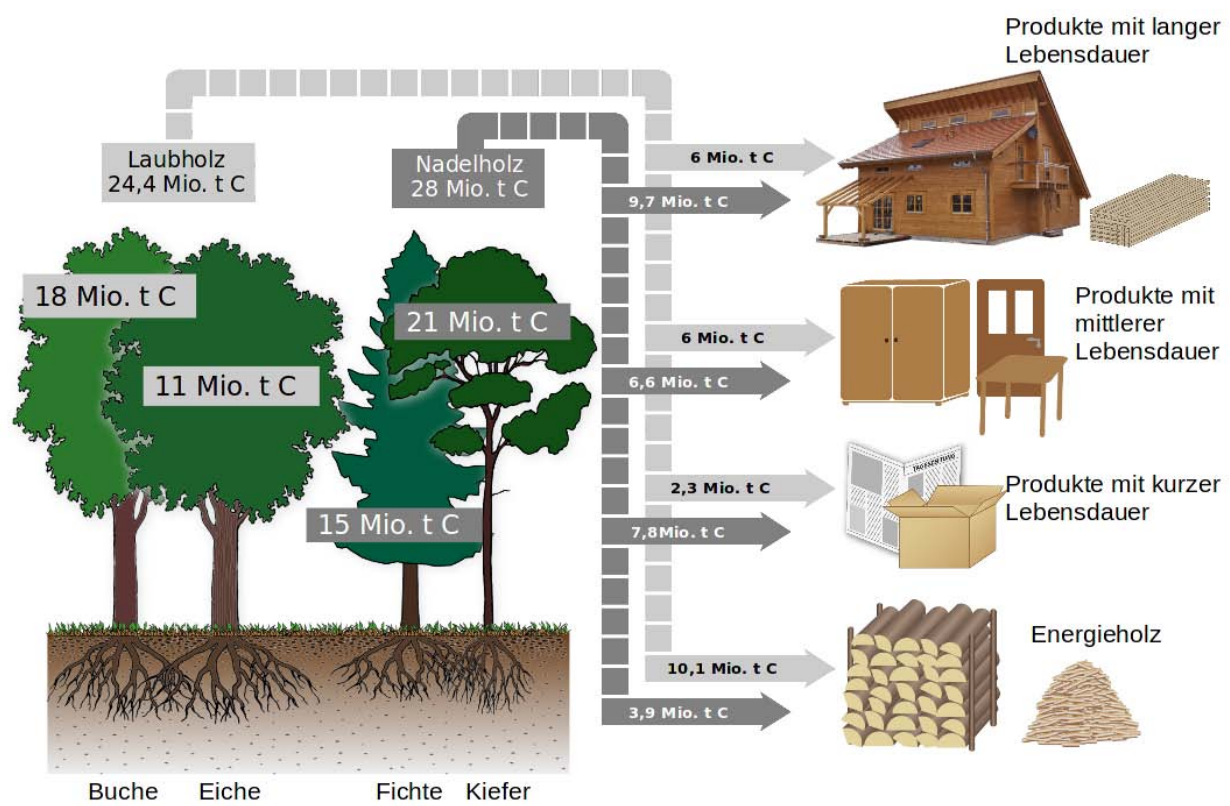

Abbildung 26: Akkumulierter C-Vorrat [Mio. tC] in der lebenden Biomasse der vier Hauptbaumarten, den Nutzungen und den vier Holaproduktklassen am Ende der Simulation des Szenarios naturnaher Waldbau im Jahre 2036 (Laubholzspeicher: hellgrau, Nadelholzspeicher: dunkelgrau)

\subsection{Carbon Forestry Management}

In dieser Studie wird gezeigt, wie sich drei unterschiedliche waldbauliche Konzepte in dreißig Jahren auf den C-Vorrat im Bestand und den daran angeschlossenen Produktpool auswirken können. Gesteuert werden die Szenarien über unterschiedliche naturschutzorientierte und eingriffsspezifische Parameter (s. Tab. 1). Sehr wirksam ist die Erhöhung bzw. Verringerung der Zielstärken auf die Vorratshaltung und die Endnutzungsmassen.

BURSCHEL et al. (1993) schlagen zur Erhöhung der C-Vorräte eine Verlängerung der Umtriebszeiten vor. Diese Maßnahme deckt sich in ihren Effekten weitgehend mit der hier simulierten Erhöhung der Zielstärke. Sie bewirkt in jedem Fall eine Anhebung der Derbholzvorräte und der in ihnen gespeicherten C-Vorräte, aber auch eine Beeinträchtigung der Liquidität der Forstbetriebe. Zudem kann sie dazu führen, dass abiotische und biotische Risiken zunehmen, Entwertungsgefahren steigen, Bestandesstrukturen verloren gehen, Lebensräume verschwinden, lichtbedürftige Verjüngung ausgedunkelt und die Versorgung der Holz- und Energiewirtschaft eingeschränkt wird.

Weitere Vorschläge von BURSCHEL et al. (1993) zur Erhöhung der C-Vorräte sind der Unterbau von Kiefernbeständen mit Schattbaumarten, die Aufforstung 
nicht bewaldeter Flächen oder der Wechsel von Kiefer zu Douglasie. Hinzu kommen positive Senkeneffekte durch Erstaufforstungen, auf die PAUL et al. (2009) hinweisen. Damit stellt sich die Frage nach der Baumartenwahl, der wichtigsten, da sehr langfristig wirkenden ökonomischen und ökologischen Entscheidung im Forstbetrieb (vgl. SPEIDEL 1972, RÖHRIG et al. 2006).

Die in dieser Studie vorgelegten Simulationsergebnisse für die Hauptbaumarten sind immer überlagert von deren Flächenanteilen, standörtlichen Zuordnungen und Altersklassenaufbau. Ein Vergleich auf der Grundlage der Gesamtwuchsleistungen der Hauptbaumarten nach den Angaben der Ertragstafelsammlung von SCHOBER (1995) für Bestände I. Bonität und mäßige Durchforstung deckt zutreffender die Unterschiede in der C-Speicherung auf. Die Derbholzvorräte wurden unter Berücksichtigung der baumartenspezifischen Raumdichten, der Biomasse-Expansionsfaktoren und einem C-Gehalt des Holzes von $50 \%$ umgerechnet. Die ertragstafelgemäße Entwicklung der C-Vorräte über dem Alter wird bestimmt vom Wachstumsgang, dem Zuwachsniveau und der Raumdichte der Hauptbaumarten (s. Abb. 27).

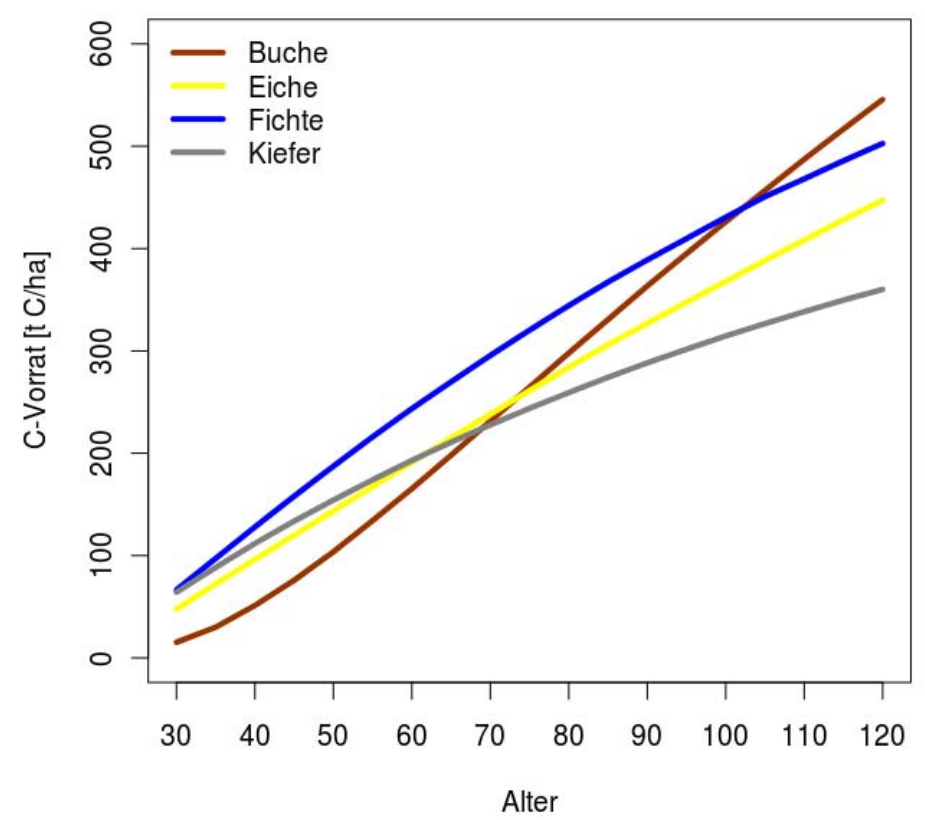

Abbildung 27: C-Vorrat [t C/ha] der lebenden Baumbiomasse der untersuchten Hauptbaumarten im Alter von 30 bis 120 Jahren auf Grundlage von Ertragstafelwerten

Die zuwachsstarke Fichte ist trotz ihrer geringeren Raumdichte zunächst allen anderen Baumarten überlegen. Erst im Alter 100, also am Ende ihres Produktionszeitraumes, wird sie von der später im Zuwachs kulminierenden Buche eingeholt. 
Diese ist zunächst auch den früh startenden Baumarten Kiefer und Eiche unterlegen. Die Eiche kann ab dem Alter 60 aufgrund ihrer höheren Raumdichte die etwas zuwachsstärkere Kiefer übertreffen und bis zum Alter 120 immer mehr zu den Baumarten Fichte und Buche aufschließen.

Noch aussagfähiger ist ein Vergleich der Baumarten auf gleichem Standort. Die Nordwestdeutsche Versuchsanstalt verfügt im Wuchsbezirk Unterer Solling über Versuche mit den Baumarten Buche, Eiche, Fichte, Douglasie und Küstentanne, die in einem jungen Alter auf vergleichbaren frischen bis vorratsfrischen, ziemlich gut versorgten Standorten angelegt wurden. Die Erstaufnahmen dieser Versuche wurden von SPELLMANN (2010) genutzt, um die Wuchsleistung und den Sortenertrag der Baumarten auf gleichem Standort und mit gleicher Durchforstungsart (starke Hochdurchforstung) mit dem Wachstumssimulator BWINPro (NAGEL et al. 2006) zu simulieren und danach die jeweilige Kohlenstoffspeicherung unter Berücksichtigung der baumartenspezifischen Raumdichten und eines C-Gehaltes des Holzes von 50 \% herzuleiten. Der Zeitraum für die Gesamtbewertung der Kohlenstoffbindung orientiert sich an dem Produktionszeitraum 200 Jahre der Eiche zur Erreichung einer Zielstärke von $75 \mathrm{~cm}$. Dies hat zur Folge, dass bei den wüchsigeren Baumarten mit kürzeren Produktionszeiträumen hierzu mehr als nur ein Umlauf in die Gesamtbilanz eingeht. Zur Vervollständigung des Bildes wurde auch ein Kiefern-Versuch I. Bonität auf einem mäßig frischen, mäßig versorgten Standort im Wuchsbezirk Hohe Heide, also einem schwächeren Standort als im Solling, in den Baumartenvergleich mit einbezogen (s. Tab 15-17).

Die Zahlen verdeutlichen die enormen Leistungsunterschiede und Ertragserwartungen bei den sechs Baumarten. Der durchschnittliche Gesamtzuwachs (dGZ) bezogen auf den jeweiligen Produktionszeitraum reicht von 7,9 $\mathrm{m}^{3} /$ ha*a bei Eiche, über 13,3 $\mathrm{m}^{3} / \mathrm{ha}^{*}$ a bei Buche bis hin zu 29,8 $\mathrm{m}^{3} /$ ha*a bei der Küstentanne. Unter den Nadelbaumarten fällt die Kiefer merklich ab, was aber auch standörtlich bedingt ist. In 52 Jahren produziert die Küstentanne ein ähnliches Volumen wie die Eiche in 200 Jahren (s. Tab. 15).

Tabelle 15: Vergleich der Wuchsleistung verschiedener Hauptbaumarten auf vergleichbarem Standort (rot umrandet: Ausnabme Kiefer) und bei gleicher Durchforstungsart

\begin{tabular}{|l|c|c|c|c|c|c|c|}
\hline Baumart & & $\mathbf{E i}$ & $\mathbf{B u}$ & $\mathbf{F i}$ & $\mathbf{D g l}$ & $\mathbf{K T a}$ & $\mathbf{K i}$ \\
\hline Produktionszeit & Jahre & 200 & 120 & 79 & 92 & 52 & 121 \\
\hline Zielstärke & $\mathrm{cm}$ & 75 & 65 & 45 & 65 & 45 & 45 \\
\hline & & & & & & & \\
\hline Nutzholz & $\mathbf{m}^{\mathbf{3} / \mathbf{h a}}$ & $\mathbf{1 4 1 7}$ & $\mathbf{1 2 6 0}$ & $\mathbf{1 1 3 0}$ & $\mathbf{1 7 3 0}$ & $\mathbf{1 4 0 1}$ & $\mathbf{8 7 4}$ \\
\hline Totholz & $\mathbf{m}^{\mathbf{3} / \mathbf{h a}}$ & $\mathbf{1 6 3}$ & $\mathbf{3 0 4}$ & $\mathbf{3 4 3}$ & $\mathbf{3 0 7}$ & $\mathbf{1 4 8}$ & $\mathbf{1 0 6}$ \\
\hline & & & & & & & \\
\hline $\mathrm{dGZ}$ & $\mathrm{m}^{3 / \mathrm{ha} / \mathbf{a}}$ & 7,9 & $\mathbf{1 3 , 3}$ & $\mathbf{1 8 , 6}$ & 22,1 & $\mathbf{2 9 , 8}$ & $\mathbf{8 , 1}$ \\
\hline
\end{tabular}


Die Sortierung der Einzelbäume führt zu wesentlich höheren Stammholzanteilen bei Fichte (66\%), Douglasie $(71 \%)$ und Kiefer $(70 \%)$ gegenüber den Laubbaumarten (48\%). Die Küstentanne (57\%) weicht diesbezüglich von den anderen Nadelbaumarten ab, weil sie sehr schnell ins Derbholz wächst und stärker differenziert ist (s. Tab. 16).

Tabelle 16: Vergleich des Sortimentsanfalls und des darin gespeicherten Koblenstoffs verschiedener Hauptbaumarten auf vergleichbarem Standort (rot umrandet: Ausnabme Kiefer) und bei gleicher Durchforstungsart (o. R. = obne Rinde)

\begin{tabular}{|c|c|c|c|c|c|c|c|c|}
\hline \multicolumn{9}{|c|}{ Baumart } \\
\hline \multirow{3}{*}{$\begin{array}{c}+1 \\
+ \\
+ \\
\text { है }\end{array}$} & Stammholz O.R. & $\mathrm{m}^{3} / \mathrm{ha}$ & 675 & 610 & 750 & 1099 & 795 & 612 \\
\hline & IN-Holz o.R. & $\mathrm{m}^{3} / \mathrm{ha}$ & 465 & 461 & 255 & 260 & 452 & 145 \\
\hline & Restholz o.R. & $\mathrm{m}^{3} / \mathrm{ha}$ & 277 & 189 & 125 & 192 & 154 & 117 \\
\hline \multirow{5}{*}{ 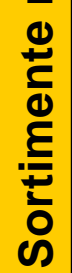 } & Stammholz o.R. & t/ha & 432 & 370 & 323 & 601 & 342 & 300 \\
\hline & IN-Holz o.R. & t/ha & 298 & 280 & 110 & 122 & 194 & 71 \\
\hline & Restholz o.R. & t/ha & 177 & 115 & 54 & 90 & 66 & 57 \\
\hline & \multirow{2}{*}{$\begin{array}{l}\text { Kohlenstoff- } \\
\text { speicher }\end{array}$} & t/ha & 453 & 383 & 244 & 407 & 301 & 214 \\
\hline & & t/ha/a & 2,265 & 3,192 & 3,089 & 4,424 & 5,788 & 1,769 \\
\hline
\end{tabular}

Durch die Umrechnung der Volumina in Massen kehren sich die Leistungsunterschiede zwischen Nadel- und Laubbaumarten um. Nur die Douglasie mit ihrer überragenden Wuchsleistung und relativ hohen Raumdichte kann in etwa mit den Laubbaumarten mithalten. Die Kohlenstoffspeicherung für den jeweiligen Produktionszeitraum der Baumarten reicht von $453 \mathrm{t} \mathrm{C} /$ ha bei Eiche bis $214 \mathrm{t} \mathrm{C} /$ ha bei Kiefer. Bezogen auf die Kohlenstoffspeicherung pro Hektar und Jahr verschieben sich die Relationen zugunsten der leistungsfähigen Nadelbaumarten. Die Fichte kann mit einer Speicherleistung von 3,089 t/ha*a fast zur Buche mit einem Wert von 3,192 t/ha*a aufschließen.

Verlässt man den Waldökosystemansatz bei der Kohlenstoffspeicherung und bezieht auch die Holzprodukte und ihre jeweilige Verweildauer im Zivilisationskreislauf mit ein, so verschieben sich die Kohlenstoffbilanzen der Baumarten weiter zugunsten der Nadelbaumarten durch den additiven Produktspeicher der Baumarten. Dieser ergibt sich aus dem Produkt der Kohlenstoffspeicherung pro Hektar und Jahr und dem Verhältnis von mittlerer Verweildauer zu mittlerem Produktionszeitraum (s. Tab. 17). 
Tabelle 17: Vergleich der Produktanteile, Verweildauern und Koblenstoffspeicherleistungen verschiedener Hauptbaumarten auf vergleichbarem Standort (rot umrandet: Ausnabme Kiefer) und bei gleicher Durchforstungsart

\begin{tabular}{|c|c|c|c|c|c|c|c|c|}
\hline \multirow{6}{*}{ 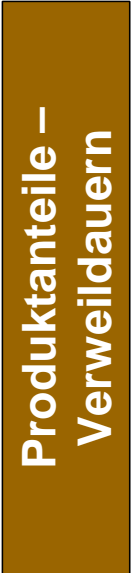 } & Forstprodukte & $\begin{array}{l}\text { Verweil- } \\
\text { dauer }\end{array}$ & $\begin{array}{c}\text { Anteil } \\
\text { Ei }\end{array}$ & $\begin{array}{c}\text { Anteil } \\
\text { Bu }\end{array}$ & $\begin{array}{c}\text { Anteil } \\
\text { Fi }\end{array}$ & $\begin{array}{c}\text { Anteil } \\
\text { Dgl }\end{array}$ & $\begin{array}{l}\text { Anteil } \\
\text { KTa }\end{array}$ & $\begin{array}{c}\text { Anteil } \\
\text { Ki }\end{array}$ \\
\hline & $\begin{array}{l}\text { Brennholz, } \\
\text { Pellets }\end{array}$ & 1 Jahr & $40 \%$ & $32 \%$ & $23 \%$ & $21 \%$ & $25 \%$ & $17 \%$ \\
\hline & $\begin{array}{l}\text { Papier, Pappe, } \\
\text { Karton }\end{array}$ & 3 Jahre & - & $20 \%$ & $10 \%$ & $10 \%$ & $11 \%$ & $11 \%$ \\
\hline & $\begin{array}{l}\text { Holzwerk- } \\
\text { stoffe, Funiere }\end{array}$ & 25 Jahre & $29 \%$ & $17 \%$ & $26 \%$ & $24 \%$ & $29 \%$ & $29 \%$ \\
\hline & \begin{tabular}{|l|} 
Bauholz, \\
Bodenbeläge
\end{tabular} & 50 Jahre & $31 \%$ & $31 \%$ & $41 \%$ & $45 \%$ & $35 \%$ & $43 \%$ \\
\hline & \begin{tabular}{|l} 
Mittlere \\
Verweildauer
\end{tabular} & Jahre & 23 & 21 & 28 & 29 & 25 & 29 \\
\hline \multirow{5}{*}{ 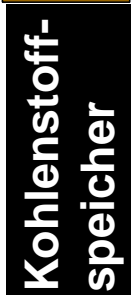 } & & & & & & & & \\
\hline & & & $\mathbf{E i}$ & $\mathrm{Bu}$ & $\mathbf{F i}$ & Dgl & KTa & $\mathbf{K i}$ \\
\hline & Kohlenstoff & t/ha/a & 2,265 & 3,192 & 3,089 & 4,424 & 5,788 & 1,769 \\
\hline & $\begin{array}{l}\text { additiver Pro- } \\
\text { duktspeicher }\end{array}$ & t/ha/a & 0,260 & 0,559 & 1,095 & 1,395 & 2,783 & 0,424 \\
\hline & $\begin{array}{l}\text { Gesamt- } \\
\text { speicher C }\end{array}$ & t/ha/a & 2,525 & 3,751 & 4,184 & 5,819 & 8,571 & 2,193 \\
\hline
\end{tabular}

Durch den einheitlichen Bewertungszeitraum wird der höhere Beitrag der wüchsigeren Baumarten zur Kohlenstoffspeicherung durch einen additiven Produktspeicher erfasst, der berücksichtigt, dass z. B. die Küstentanne während des 200jährigen Produktionszeitraumes der Eiche ihren Produktspeicher fast viermal auffüllt. In der Gesamtbilanz bedeutet dies, dass die Gesamtspeicherleistungen der Baumarten von 2,193 t C/ha*a bei Kiefer, über 3,751 bzw. 4,184 t C/ha*a bei Buche bzw. Fichte bis 5,819 bzw. 8,571 t C/ha*a bei Douglasie bzw. Küstentanne reichen. Berücksichtigt man zudem noch, dass in Nadelwaldbeständen der Kohlenstoffspeicher deutlich höher als in Laubwälder ist, so kann man mit der Baumartenwahl die Kohlenstoffspeicherung im Wald und in den Holzprodukten wesentlich beeinflussen. 


\section{Schlussfolgerungen und Ausblick}

Das Prinzip der multifunktionalen Nachhaltigkeit ist das Grundprinzip einer modernen Forstwirtschaft in Niedersachsen. Es wurde zuletzt 2010 durch das Strategiepapier der Landesregierung „Wälder für Niedersachsen - Wald, Forst- und Holzwirtschaft im Wandel“" bekräftigt und von zahlreichen Verbänden gegengezeichnet. Dieses Prinzip lässt sich erst dann umsetzen, wenn angegeben wird, für welche Zustände, Wirkungen und Leistungen des Waldes Kontinuität bzw. Verbesserung gefordert werden (SPELLMANN et al. 2001). Einen wesentlichen Anhalt für diese Konkretisierung bietet der Katalog der gesamteuropäischen Kriterien und Indikatoren, der 2003 auf der Ministerkonferenz zum Schutz der Wälder in Europa in Wien verabschiedet wurde (MCPFE 2003). Dieser Katalog umfasst 6 Kriterien und 35 quantitative Indikatoren. Ein Indikator ist auch die Kohlenstoffspeicherung in Wäldern. Dieser Indikator hat durch die Diskussionen über die Folgen des Klimawandels und die Maßnahmen zu ihrer Begrenzung in den letzten Jahren stark an Bedeutung gewonnen. Die Kohlenstoffspeicherung wird als Teilziel der Forstbetriebe in Zukunft ein höheres Gewicht erhalten. Sie darf aber dennoch nicht isoliert gesehen werden, sondern muss in den Zusammenhang mit den anderen Regelungsfunktionen (ökosysteminterne Kreisläufe), Lebensraumfunktionen (Biodiversität), Nutzfunktionen (Holz, Wasser, Jagd) sowie den Kultur- und Sozialfunktionen (Arbeit, Einkommen, Kulturerbe, Erholung) der Wälder gestellt werden (BEESE 1996).

Mit der Kohlenstoffstudie Forst und Holz Niedersachsen greift die niedersächsische Landesregierung dieses klimapolitisch hoch aktuelle Thema auf. Als Grundlage für notwendige Weichenstellungen werden in dieser Studie die Mengen an Kohlenstoff hergeleitet, die heute bzw. künftig in niedersächsischen Wäldern und in den aus niedersächsischem Holz hergestellten Produkten gespeichert werden. Insgesamt zeigt sich, dass der niedersächsische Wald und die aus ihm hervorgegangenen Holzprodukte einen wichtigen Beitrag zur C-Speicherung leisten und dass die Speichermengen durch die Strategien der Forst- und Holzwirtschaft merklich beeinflusst werden können. Im Sinne des Carbon Forestry Management (JARVIS et al. 2005) und des Vorschlags des wissenschaftlichen Beirats der Bundesregierung Globale Umweltfragen (WBGU 2009) empfiehlt sich von den überprüften Waldbaukonzepten die naturnahe Variante. Sie bleibt dem Grundprinzip einer nachhaltigen, multifunktionalen Forstwirtschaft treu, erhält die C-Vorräte im Wald nachhaltig auf einem hohen Niveau und erhöht zusätzlich die Senkenleistung, indem sie einen ähnlich hohen Beitrag zur C-Sequestrierung im Produktpool leistet wie das ertragsorientierte Szenario (s. Abb. 25). Letzteres verringert durch stärkere Eingriffe die C-Vorräte in der lebenden Baumbiomasse der Bestände auf ein wesentlich niedrigeres Niveau und fördert gleichzeitig durch erhöhte Bodentemperaturen die Freisetzung von C aus dem Boden (vgl. JANDL et al. 2007). Beim naturschutz- 
orientierten Szenario werden im dreißigjährigen Simulationszeitraum die höchsten C-Vorräte akkumuliert. Danach wird aber die Senkenleistung bei gleichbleibender Behandlung immer geringer, da der Volumenzuwachs nachlässt oder die aufgeschobenen Eingriffe zeitlich verzögert durchgeführt werden, wodurch es zu einem Abbau der Kohlenstoffvorräte im lebenden Bestand kommt.

Der Baumartenvergleich hat noch einmal die Bedeutung des Nadelholzes für die Kohlenstoffspeicherung unterstrichen. Verlässt man den Waldökosystemansatz und bezieht auch die Holzprodukte und ihre jeweilige Verweildauer im Zivilisationskreislauf mit ein, so verschieben sich die Kohlenstoffbilanzen eindeutig zugunsten der leistungsfähigen Nadelbaumarten. Eine Sicherung des Nadelholzanbaus deckt sich somit nicht nur mit den ökonomischen Zielen der Forstbetriebe und der Nachfrage der Holzindustrie, sondern fördert ebenso die Kohlenstoffbindung im Wald und in Holzprodukten. Dies gilt besonders für das Bauholz mit seiner langen Verweildauer. Ungeachtet der Baumart ist es aber für die deutsche Forstwirtschaft allgemein wichtig, dass die Kohlenstoffspeicherung in Holzprodukten in die Kohlenstoffbilanzen mit einbezogen und entsprechend bewertet wird. Andernfalls würden die seit langem nachhaltig wirtschaftenden Forstbetriebe benachteiligt, wenn sie nach dem Erreichen der jeweils angestrebten Zielvorräte keinen weiteren Vorratsaufbau mehr zulassen und entsprechend die Nutzungen anheben. Hier sind die politischen Entscheidungsträger gefragt, diese Option offiziell einzufordern. 


\section{Literatur}

3N (2010): Feuerstättenzählung Niedersachsen 2009 für holzbefeuerte Anlagen bis 1 MW. 3N - Kompetenzzentrum Niedersachsen Netzwerk Nachwachsende Rohstoffe in Zusammenarbeit mit dem Landesinnungsverband für das Schornsteinfegerhandwerk Niedersachsen (LIV) im Auftrag des Niedersächsischen Ministeriums für Ernährung, Landwirtschaft, Verbraucherschutz und Landesentwicklung, Werlte, $101 \mathrm{~S}$.

AG EnERGIEBILANZEN E.V. (2010): Evaluation Tables on the Energy Balance for the Federal Republic of Germany 1990 to 2009.

www.ag-energiebilanzen.de/viewpage.php?

AWGPK (2010): Consideration of further commitments for Annex I Parties under the Kyoto Protocol. Ad Hoc Working Group on Further Commitments for Annex I Parties under the Kyoto Protocol. Fifteenth session, Cancun, 29 November.

http://unfccc.int/documentation/documents/advanced_search/items/3594.p hp? $\mathrm{rec}=$ j\&priref $=600006112 \&$ data $=\&$ title $=\&$ author $=\&$ keywords $=\&$ symbol $=$ \&meeting $=\% 22 \mathrm{Ad}+\mathrm{Hoc}+$ Working + Group + on + Further + Commitments + for + Annex $+\mathrm{I}+$ Parties + under + the + Kyoto + Protocol $+\% 28 \mathrm{AWG}$ -

$\mathrm{KP} \% 29 \% 2 \mathrm{C}+$ Fifteenth + session $\% 2 \mathrm{C}+29+$ November+to $+10+$ December +20 $10 \% 2 \mathrm{C}+$ Cancun $\% 2 \mathrm{C}+$ Mexico $\% 22 \&$ mo_from $=$ \&year_from $=\&$ mo_to $=\& y e a r$ _to $=\&$ last_days $=\& a n f=0 \&$ sorted $=$ date_sort\&dirc $=$ DESC\&seite $=\#$ beg Zugriff am 05.05.2011

BARTELINK, H. H. (1997): Allometric relationships for biomass and leaf area of beech (Fagus sylvatica L). Annales des Sciences Forestieres, 54, 39-50

Bauer, G. A.; Persson, H.; Perrson, T.; Mund, M.; Hein, M.; Kummetz, E.; MatTeucci, G.; Oene, V. H.; Scarascia-MugnozZA, G. u. Schulze, E.-D. (2000): Linking Plant Nutrition and Ecosystem Processes. In: SCHULZE, E.-D. (Hrsg.): Carbon and Nitrogen Cycling in European Forest Ecosystems. Springer Verlag, Berlin, 63-98

BEESE, F.O. (1996): Indikatoren für eine multifunktionelle Waldnutzung. Forstw. Cbl., 115, 65-79

BMELV (2005): Die zweite Bundeswaldinventur - Der Inventurbericht. Bundesministerium für Ernährung, Landwirtschaft und Verbraucherschutz, Referat 533, Bonn, $231 \mathrm{~S}$. 
BMELV (2008): Holzmarktbericht 2007. Abschlussergebnisse für die Forst- und Holzwirtschaft des Wirtschaftsjahres 2007. Bundesministerium für Ernährung, Landwirtschaft und Verbraucherschutz http://www.bmelv-statistik.de/de/fachstatistiken/forst-und-holzwirtschaft/

BMELV (2009): Holzmarktbericht 2008. Bundesministerium für Ernährung, Landwirtschaft und Verbraucherschutz. http://www.bmelv.de

BÖSWALD, K. (1996): Zur Bedeutung des Waldes und der Forstwirtschaft im Kohlenstoffhaushalt, eine Analyse am Beispiel des Bundeslandes Bayern. Forstliche Forschungsberichte München, 159, 147 S.

BÖSWALD, K. u. WIERLING, R. (1997): Wald und Forstwirtschaft Niedersachsens im Kohlenstoffhaushalt. In: NIEDERSÄCHSISCHES MINISTERIUM FÜR ERNÄHRUNG, LANDWIRTSCHAFT U. FORSTEN (Hrsg.): Aus dem Walde, 50, Mitteilungen der Niedersächsischen Landesforstverwaltung, Teil II, 247-333

Bolte, A.; Rahmann, T.; Kuhr, M.; Pogoda, P.; Murach, D. u. Gadow, K. (2004): Relationships between tree dimension and coarse root biomass in mixed stands of European beech (Fagus sylvatica L.) and Norway spruce (Picea abies [L.] Karst.). Plant and Soil, 264, 1-11

Burschel, P.; Kürsten, E. u. Larson, B. C. (1993): Die Rolle von Wald und Forstwirtschaft im Kohlenstoffhaushalt - Eine Betrachtung für die Bundesrepublik Deutschland. Schriftenreihe der Forstwissenschaftlichen Fakultät der Universität München und Bayerischen Forstlichen Versuchs- und Forschungsanstalt, 126, $135 \mathrm{~S}$.

Cienciala, E.; Cerny, M.; Apltauer, J. u. Exnerová, Z. (2005): Biomass functions applicable to European beech. Journal of Forest Science, 51, 147-154

Cienciala, E.; Černy, M.; TAtarinov, F.; Apltauer, J. u. Exnerová, Z. (2006): Biomass functions applicable to Scots pine. Trees - Structure and Function, 20, 483-495

Cienciala, E.; Apltauer, J.; Exnerová, Z. u. TAtarinov, F. (2008): Biomass functions applicable to oak trees grown in Central-European forestry. Journal of Forest Science, 54, 109-120

DIETER, M. u. ELSASSER, P. (2002): Carbon stocks and carbon stock changes in the tree biomass of Germany's forests. Forstwissenschaftliches Centralblatt, 121, $195-210$

DORMANN, F. u. KÜHN, I. (2009): Angewandte Statistik für die biologischen Wissenschaften. Helmholtz-Zentrum für Umweltforschung (UFZ), Halle, $257 \mathrm{~S}$. 
Dunger, K.; StÜMER, W.; OEHMiChen, K.; Riedel, T. u. BOLTE, A. (2009): Der Kohlenstoffspeicher Wald und seine Entwicklung. AFZ/Der Wald, 64, 20, 1072-1073

EllenberG, H.; MAYER, R. u. SCHAUERMAnN, J. (1996): Ökosystemforschung Ergebnisse des Sollingprojektes. Ulmer Verlag Stuttgart, 507 S.

FIEDLER, F. (1986): Die Dendromasse eines hiebsreifen Fichtenbestandes. Beiträge für die Forstwirtschaft, 20, 171-180

Fraver, S.; WAGNER, R. \& DAY, M. (2002): Dynamics of coarse woody debris following gap harvesting in the Acadian forest of central Maine, USA. Can. J. For. Res., 32, 2094-2105

FrÜHWALD, A.; Diederichs, S. u. MORGAN, R. M. (2010): Verwendungspotentiale heben durch Kaskadennutzung am Beispiel Holz. In: THOMÉKOZMIENSKY, K. J. u. BECKMANN, M. (Eds.): Erneuerbare Energien, Band 4 Biomasse und Biogas, Ersatzbrennstoffe, Solar- und Windenergie. TK Verlag Karl Thomé-Kozmiensky Neuruppin, 37-50

HASENAUER, H. (2011): Überlegungen zur $\mathrm{CO}_{2}$-Bilanz von Waldökosystemen. Austrian J. For. Sci., 128, 33-52

HEINSDORF, D. u. KRAUß, H. H. (1990): Schätztafeln für Trockenmasse und Nährstoffspeicherung von Kiefernbeständen. Institut für Forstwissenschaften, Eberswalde, DDR, $77 \mathrm{~S}$.

Hessenmöller, D.; Schulze, E.-D. u. GroßmanN, M. (2008): Bestandesentwicklung und Kohlenstoffspeicherung des Naturwaldes „Schönstedter Holz“ im Nationalpark Hainich. Allg. Forst- und Jagdztg., 179, 209-2219

HEUER, E. (2010): Was hat sich in Kopenhagen bewegt? AFZ/Der Wald, 65, 3, 4 5

HoCHBICHLER, E. (2002): Vorläufige Ergebnisse von Biomasseninventuren in Buchen-und Mittelwaldbeständen. In: DiETRICH, H.-P.; RASPE, S. u. PREUHSLER, T. (Eds.): Inventur von Biomasse- und Nährstoffvorräten in Waldbeständen. Forstliche Forschungsberichte München, LWF München, 186, $37-46$

ILG, S. (2002): Erhebung der oberirdischen Biomasse- und Elementvorräte eines Fichtenbestandes (Picea abies (L.) Karst) im Bereich der Waldklimastation Zusmarshausen. Diplomarbeit, Fachhochschule Weihenstephan, Fachbereich Forstwirtschaft, $100 \mathrm{~S}$.

IPCC (2007): Climate Change 2007: Synthesis Report. Contribution of Working Groups I, II and III to the Fourth Assessment Report of the Intergovernmental Panel on Climate Change. Geneva, IPCC 
Jacobsen, C.; Rademacher, P.; Meesenburg, H. u. Meiwes, K. J. (2003): Gehalte chemischer Elemente in den Baumkompartimenten - Literaturstudie und Datensammlung. Berichte des Forschungszentrums Waldökosysteme der Universität Göttingen, Reihe B, Band 69, 92 S.

JANDL, R.; LindNER, M.; VESTERDAL, L.; BAUWENS, B.; BARITZ, R.; HAGEDORN, F.; Johnson, D. W.; MinkKinen, K. u. BYRne, K. A. (2007): How strongly can forest management influence soil carbon sequestration? Geoderma, 137, $253-268$

JARVIS, P. G.; IBROM, A. u. LINDER, S. (2005): Carbon forestry: managing forests to conserve carbon. In: GRIFfithS, H. u. JARVIS, P. G. (Hrsg.): The Carbon Balance of Forest Biomes. Taylor \& Francis Group Oxford, 331-349

JoOsten, R.; SCHumacher, J.; WirTh, C. u. SCHUlte, A. (2004): Evaluating tree carbon predictions for beech (Fagus sylvatica L.) in western Germany. Forest Ecology and Management, 189, 87-96

KNIGGE, W. u. SchUlZ, H. (1966): Grundriss der Forstbenutzung. Verlag Paul Parey, Hamburg u. Berlin, $584 \mathrm{~S}$.

KÖHL, M.; FrÜHWALD, A.; KeNTER, B.; OlsChOfSKY, K.; KÖHLER, R.; KÖTHKE, M.; RÜTer, S.; Pretzsch, H.; RÖTZER, T.; MAKeschin, F.; ABIY, M. u. DieTER, M. (2009): Potenzial und Dynamik der Kohlenstoffspeicherung in Wald und Holz: Beitrag des deutschen Forst- und Holzsektors zum Klimaschutz. vTI Agriculture and Forestry Research, Sonderheft 327, 103-109

KÖNIG, N. u. FORTMANN, H. (1996): Probenvorbereitungs-, Untersuchungs- und Elementbestimmungsmethoden des Umweltanalytik-Labors der Niedersächsischen Forstlichen Versuchsanstalt und des Zentrallabors II des Forschungszentrums Waldökosysteme. Teil 3: Probenvorbereitungs- und Untersuchungsmethoden, Qualitätskontrolle und Datenverarbeitung. Berichte des Forschungszentrums Waldökosysteme, Reihe B, Band 49

KÖNIG, N., FORTMANN, H. u. LÜTER, K.-L. (2009): Probenvorbereitungs-, Untersuchungs- und Elementbestimmungsmethoden des Umweltanalytik-Labors der Nordwestdeutschen Forstlichen Versuchsanstalt. 2. Ergänzung: 1999-2008. Berichte des Forschungszentrums Waldökosysteme, Reihe B, Bände 75-78

KORPEL, S. (1995): Die Urwälder der Westkarpaten. Gustav Fischer Verlag, Stuttgart, Jena, New York

Kramer, H. u. AKCA, A. (2008): Leitfaden zur Waldmesslehre. J. D. Sauerländer's Verlag Frankfurt am Main, 5. überarbeitete Auflage, 226 S.

KRUG, J. (2010): Bewertung der deutschen Forstwirtschaft in der Klimapolitik. AFZ/Der Wald, 65, 17, 30-33 
LE GOFF, N. u. OTTORINI, J. M. (2001): Root biomass and biomass increment in a beech (Fagus sylvatica L.) stand in North-East France. Annals For. Sci., 58, 1-13

LOREnZ, K. u. LAL, R. (2010): Carbon Sequestration in Forest Ecosystems. Springer Verlag Dordrecht, 279 S.

Luyssaert, S.; Inglima, I.; Jung, M.; RiChardson, A. D.; ReICHSTEIN, M.; Papale, D.; PiaO, S. L.; Schulze, E.-D.; Wingate, L.; MatTeucci, G.; Aragao, L.; Aubinet, M.; Beer, C.; Bernhofer, C.; BlacK, K. G.; BOnal, D.; Bonnefond, J. -M.; Chambers, J.; Ciais, P.; COOK, B.; DAvis, K. J.; DOlman, A. J.; Gielen, B.; GOUlden, M.; GraCE, J.; GrAnier, A.; Grelle, A.; Griffis, T.; GrÜNWALD, T.; GUIDOlOtTI, G.; HANSON, P. J.; HARDING, R.; Hollinger, D. Y.; Hutyra, L. R.; Kolari, P.; Kruijt, B.; KuTSCH, W.; Lagergren, F.; LAUrila, T.; LAW, B. E.; LE MAIRE, G.; Lindroth, A.; Loustau, D.; Malhi, Y.; Mateus, J.; Migliavacca, M.; Misson, L.; Montagnani, L.; MOnCRIEFF, J.; MOORS, E.; MungER, J. W.; NiKINMAA, E.; Ollinger, S. V.; PitA, G.; Rebmann, C.; Roupsard, O.; SAIGUSA, N.; SANZ, M. J.; SEUfERT, G.; SIERRA, C.; SMITH, M.-L.; TANG, J.; VALENTINI, R.; VESALA, T.; JANSSENS, I. A. (2007): $\mathrm{CO}_{2}$ balance of boreal, temperate, and tropical forests derived from a global database. Global Change Biology, Vol. $13,12,2509-2537$

Luyssaert, S.; SChulze, E.-D.; BÖRnER, A.; KNOHL, A.; HessenMÖller, D.; LAW, B. E.; CiAIS, P. u. GRACE, J. (2008): Old-growth forests as global carbon sinks. Nature, 455, 213-215

Mackensen, J.; BAuHus, J. u. WeBber, E. (2003): Decomposition rates of coarse woody debris - A review with particular emphasis on Australian tree species. Australian J. Botany, 51, 27-37

ManTau, U. (2004): Holzrohstoffbilanz für Deutschland - Holzrohstoffaufkommen und dessen Verwendung im Jahr 2002. Holz-Zentralblatt, 76, 10261028

ManTau, U. (2008): Holzrohstoffbilanz Deutschland, Szenarien des Holzaufkommens und der Holzverwendung bis 2012. Hamburg: INFRO - Informationssysteme für Rohstoffe und Universität Hamburg, Zentrum Holzwirtschaft, 2008, $79 \mathrm{~S}$.

Mantau, U.; SaAl, U.; Prins, K.; Steierer, F.; Linder, M.; Verkerk, H.; Eggers, J.; LeEK, N.; Oldenburger, J.; Asikainen, A. u. AntTila, P. (2010): EUwood - Real potential for changes in growth and use of EU forests. Final report. Hamburg/Germany, June 2010, 160 S.

MCPFE 2003: Improved Pan-European Indicators for Sustainable Forest Management. MCPFE Liason Unit Vienna, www.mcpfe.org 
MEYER, P., LÜPKE, B. V. u. TABAKU, V. (2003): Die Struktur albanischer Urwälder - Ableitungen für eine naturnahe Buchenwirtschaft. Forstwissenschaftliches Centralblatt, 122, 47-58

Meyer, P.; Menke, N.; Nagel, J.; Hansen, J.; Kawaletz, H.; PaAr, U. u. EvERS, J. (2009): Entwicklung eines Managementmoduls für Totholz im Forstbetrieb. Nordwestdeutsche Forstliche Versuchsanstalt, Abschlussbericht, Deutsche Bundesstiftung Umwelt, $110 \mathrm{~S}$.

ML - NIEDERSÄCHSISCHES MINISTERIUM FÜR DEN LÄNDLICHEN RAUM, ERNÄHRUNG, LANDWIRTSCHAFT UND VERBRAUCHERSCHUTZ (2004): Der Wald in Niedersachsen - Ergebnisse der BWI². Aus dem Walde - Schriftenreihe Waldentwicklung in Niedersachsen, 55, $48 \mathrm{~S}$.

ML - NIEDERSÄCHSISCHES MINISTERIUM FÜR ERNÄHRUNG, LANDWIRTSCHAFT, VERBRAUCHERSCHUTZ UND LANDESENTWICKLUNG (2010): Wälder für Niedersachsen - Wald, Forst- und Holzwirtschaft im Wandel. Broschüre, 36 S.

MuChIN, A.; BILKe, G.; Murn, Y. u. BÖGE, R. (2008): Nachhaltige Potenziale von Dendromasse. In: MurACH, D., KNur, L. u. SCHulTZE, M. (Hrsg.): DENDROM - Zukunftsrohstoff Dendromasse. Verlag Dr. Norbert Kessel, 117-149

MÜller-Using, S. u. BARTSCH, N. (2009): Decay dynamic of coarse and fine woody debris of a beech (Fagus sylvatica L.) forest in Central Germany. Europ. J. For. Res., 128, 287-296

Mund, M. (2004): Carbon pools of European beech forests (Fagus sylvatica) under different silvicultural management. Dissertation, Forschungszentrum Waldökosysteme der Universität Göttingen, 263 S.

Mund, M.; ProffT, I.; Wutzler, T.; SChulze, E.-D.; WeBER, G. u. Weller, E. (2006): Vorbereitungen auf eine laufende Fortschreibung der Kohlenstoffvorräte in den Wäldern Thüringens. Mitteilungen / Thüringer Landesanstalt für Wald, Jagd und Fischerei, 26, 128 S.

MUUKKONEN, P. (2007): Generalized allometric volume and biomass equations for some tree species in Europe. European Journal of Forest Research, 126, 157-166

Nabuurs, G. J.; Thürig, E.; Heidema, N.; Armolaitis, K.; Biber, P.; Cienciala, E.; Kaufmann, E.; MäkipÄÄ, R.; Nilsen, P.; Petritsch, R.; Pristova, T.; Rock, J.; SchelhaAs, M. -J.; Sievanen, R.; SOMOGYI, Z. u. VALLET, P. (2008): Hotspots of the European forests carbon cycle. Forest Ecology and Management, 256, 194-200

NAGEL, J.; DUDA, H.; HANSEN, J. (2006): Forest Simulator BWINPro7. Forst u. Holz, 61, 427-429 
NFP - NiEDERSÄCHSISCHES FORSTPLANUNGSAMT (2004): Langfristige ökologische Waldentwicklung - Richtlinie zur Baumartenwahl. Aus dem Walde, 54. Niedersächsisches Ministerium für den ländlichen Raum, Ernährung, Landwirtschaft und Verbraucherschutz, $150 \mathrm{~S}$.

Oehmichen, K.; Demant, B.; Dunger, K.; Grüneberg, E.; Hennig, P.; Kroiher, F.; Neubauer, M.; Polley, H.; Riedel, T.; Rock, J.; SchwitzGebel, F.; StÜMer, W.; Wellbrock, N.; Ziche, D. u. Bolte, A. (2011): Inventurstudie 2008 und Treibhausgasinventar Wald. Johann Heinrich von Thünen-Institut (vTI), Landbauforschung, Sonderheft 343

Отто, H.-J. (1972): Die Ergebnisse der Standortkartierung im pleistozänen Flachland Niedersachsens - Grundlage waldbaulicher Vorstellungen. Aus dem Walde - Mitteilungen aus der Niedersächsischen Landesforstverwaltung, 19

PAul, C.; Weber, M. u. MOSAndL, R. (2009): Kohlenstoffbindung junger Aufforstungssflächen. Karl Gayer Institut in Zusammenarbeit mit dem Lehrstuhl für Waldbau der Technichen Universtät München, $70 \mathrm{~S}$.

PELLINEN, P. (1986): Biomasseuntersuchungen im Kalkbuchenwald. Dissertation, Fakultät für Forstwissenschaften und Waldökologie, Georg-August-Universität Göttingen, 145 S.

PfLÜGER-GRONE, H. (2007): Energieträger Holz - Ein umweltverträglicher und ökologischer Brennstoff. Broschüre des Kompetenzzentrums HessenRohstoffe (HeRo) e.V., Witzenhausen

PISTORIUS, T. (2007): Die Bedeutung von Kohlenstoffbilanzen im Diskurs über die Einbindung der Forstwirtschaft in die nationale Klimapolitik. Dissertation, Fakultät für Forst- und Umweltwissenschaften der Albert-Ludwigs-Universität zu Freiburg im Breisgau, 255 S.

POEPPEL, B. (1989): Untersuchungen der Dendromasse in mittelalten Fichtenbeständen. Diplomarbeit, Forsteinrichtung und Forstliche Ertragskunde, Technische Universität Dresden, 66 S.

Polley, H.; Henning, P. u. Kroiher, F. (2009): Baumarten, Altersstruktur und Totholz in Deutschland. AFZ/Der Wald, 64, 20, 1074-1075

Pregitzer, K. u. EuskirCHEN, E. (2004): Carbon cycling and storage in world forests: biome patterns related to forest age. Global Change Biology, Vol. 10, 12, 2052-2077

PretzsCH, H. (2000): Die Regeln von Reineke, Yoda und das Gesetz der räumlichen Allometrie. Allg. Forst- und Jagdztg., 171, 205-210

PretzsCH, H. (2009): Forest Dynamics, Growth and Yield: From Measurement to Model. Springer-Verlag Berlin, Heidelberg, 664 S. 
PROFFT, I. (2010): Holzprodukte für den Klimaschutz - Der aktuelle Trend in Thüringen. Forst u. Holz, 65, 10, 18-23

Profft, I.; Mund, M.; Weber, G. E.; Weller, E. u. Schulze, E.-D. (2009): Forest management and carbon sequestration in wood products. Europ. J. For. Res., 128, 399-413

RAdemacher, P.; KhannA, P. K.; EICHhORn, J. u. Guericke, M. (2009): Tree Growth, Biomass, and Elements in Tree Components of Three Beech Sites. In: BRumme, R. u. KHANNA, P. K. (Hrsg.): Functioning and Management of European Beech Ecosystems. Springer, 105-136

Ranius, T.; Kindvall, O.; Kruys, N. \& JOnsson, B. (2003): Modelling dead wood in Norway spruce stands subject to different management regimes. Forest Ecology and Management, 182, 13-29

ROBINSON, D. (2007): Implications of a large global root biomass for carbon sink estimates and for soil carbon dynamics. Proc. R. Soc. B, 274, 2753-2759

ROCK, J. (2008): Klimaschutz und Kohlenstoff in Holz: Vergleich verschiedener Strategien. Dissertation, Mathematisch-Naturwissenschaftliche Fakultät der Universität Potsdam, $162 \mathrm{~S}$.

ROCK, J.; BADECK, F. W. u. HARMON, M. E. (2008): Estimating decomposition rate constants for European tree species from literature sources. Europ. J. For. Res., 127, 301-313

RÖHRIG, E.; BARTSCH, N. u. LÜPKE, B. V. (2006): Waldbau auf ökologischer Grundlage. Verlag Eugen Ulmer Stuttgart, 7. Auflage, 479 S.

RÜTER, S. (2010): Einbeziehung von Holzprodukten in die Klimapolitik. HolzZentralblatt, 136, 25, 623-624

RÜTHer, B.; Hansen, J.; Ludwig, A.; Spellmann, H.; NAGEl, J.; MÖHRing, B. u. Dieter, M. (2007): Clusterstudie Forst und Holz Niedersachsen. Beiträge aus der Nordwestdeutschen Forstlichen Versuchsanstalt, Band 1, Universitätsverlag Göttingen, $92 \mathrm{~S}$.

SACHS, L. u. HedDERICH, J. (2006): Angewandte Statistik. Springer, Berlin Heidelberg, New York, 702 S.

SAUGIER, B.; RoY, J. u. MOONEY, H.A. (2001): Estimates of global terrestrial productivity: converging toward a single number. In: ROY, J.; SAUGIER, B. u. MoOney, H.A. (Eds): Terrestrial global productivity. Academic, San Diego, CA, 543-557

SCHOBER, R. (1995): Ertragstafeln wichtiger Baumarten bei verschiedener Durchforstung. Neubearb. v. R. Schober, 4. Aufl., Frankfurt am Main, Sauerländer Verlag, 166 S. 
Schulze, E.-D.; Wirth, C. u. Heimann, M. (2002): Carbon Fluxes of the Eurosiberian Region. Environ. Control in Biol., 40, 3, 249-258

SCHWARZMEIER, M. (2000): Erhebung der oberirdischen Biomassevorräte von Fichtenbeständen (Picea abies (L.) Karst) im Bereich der Waldklimastationen Ebersberg und Flossenbürg. Diplomarbeit, Fachhochschule Weihenstephan Fachbereich Forstwirtschaft, 155 S.

Somogyi, Z.; Cienciala, E.; MÄKIPÄÄ, R.; MuUkKOnen, P.; LEHTONEN, A. u. WEISS, P. (2007): Indirect methods of large-scale forest biomass estimation. European Journal of Forest Research, 126, 197-207

SPEIDEL, G. (1972): Planung im Forstbetrieb. Hamburg u. Berlin, 267 S.

SPELlmanN, H. (2010): Waldbauliche Möglichkeiten zur Sicherung der Nadelrohholzversorgung. Vortrag im Rahmen der Tagung: Sicherung der Nadelrohholzversorgung. Kompetenznetz für Nachhaltige Holznutzung (NHN e. V.), 12.11.2010, Göttingen.

http://www.kompetenznetz-holz.de/aktuelles/nadelholz/8_Spellmann.pdf

SpellmanN, H.; Hillebrand, K. u. CORnElius, P. (2001): Konzept zur Erfassung und Sicherung der Nachhaltigkeit in multifunktional genutzten Wäldern, Forst u. Holz, 56, 469-473

SPellmanN, H.; KeHr, I.; HilleBrand, K.; SCHÜtZE, C.; PrETZSCH, H.; POTT, M.; BitTer, A. W.; Koffinke, B.; Bredemeier, M. u. SCHulte-Bisping, H. (2004): Nachhaltige und multifunktionale Forstwirtschaft - ein Verfahrens- und Zustandsvergleich für verschiedene Testregionen in der Bundesrepublik Deutschland. STOCK, R. (Hrsg.). Schriftenreihe "Initiativen zum Umweltschutz" der Deutschen Bundesstiftung Umwelt (DBU), Band 58, ErichSchmidt-Verlag, $283 \mathrm{~S}$.

TABAKU, V. u. MEYER, P. (1999): Lückenmuster in Buchenwäldern unterschiedlicher Nutzungsintensität. Forstarchiv, 70, 87-97

Taverna, R.; Hofer, P.; Werner, F.; Kaufmann, E. U. Thürig, E. (2007): $\mathrm{CO}_{2}$-Effekte der Schweizer Wald- und Holzwirtschaft. Szenarien zukünftiger Beiträge zum Klimaschutz. Umwelt-Wissen Nr. 0739. Bundesamt für Umwelt, Bern, $102 \mathrm{~S}$.

UMWELTBUNDESAMT (2010a): Nationaler Inventarbericht zum Deutschen Treibhausgasinventar 1990 - 2008. Umweltbundesamt, Dessau-Roßlau, 675 S.

UMWELTBUNDESAMT (2010b): Klimaneutral leben - Verbraucher starten durch beim Klimaschutz. Umweltbundesamt, Dessau-Roßlau, 28 S.

VDP (2007): Papier - Ein Leistungsbericht. Verband Deutscher Papierfabriken e.V., Bonn, $85 \mathrm{~S}$. 
WBGU (1998): Die Anrechnung biologischer Quellen und Senken im KyotoProtokoll: Fortschritt oder Rückschlag für den globalen Umweltschutz. Wissenschaftlicher Beirat der Bundesregierung Globale Umweltveränderungen. WBGU Bremerhaven, Sondergutachten 1998, 76 S.

WBGU (2009): Welt im Wandel - Zukunftsfähige Bioenergie und nachhaltige Landnutzung. Wissenschaftlicher Beirat der Bundesregierung Globale Umweltveränderungen. WBGU Berlin, $388 \mathrm{~S}$.

WeIS, W. u. GÖTTLEIN, A. (2002): Vergleich von Biomasse, Elementgehalten und Elementvorräten von Fichte (Picea abies L. Karst.) und Buche (Fagus sylvatica L.) am Standort Höglwald zu Zeiten der Vegetationsruhe. In: DIETRICH, H.-P., RASPE, S. u. PREUHSLER, T. (Hrsg.): Inventur von Biomasse- und Nährstoffvorräten in Waldbeständen. Forstliche Forschungsberichte München, 186, $163-167$

Wellbrock, N.; Aydin, C.-T.; Block, J.; Bussian, B.; Deckert, M.; DieKmanN, O.; Evers, J.; Fetzer, K. D.; GAuer, J.; Gehrmann, J.; KÖlling, C.; KÖNIG, N.; LIESEBACH, M.; MARTIN, J.; MeIWES, K. J.; Milbert, G.; RABEN, G.; RIEK, W.; SCHÄFFER, W.; SCHWERHOFF, J.; ULLRICH, T.; UTERMANN, J.; VOlZ, H.-A.; WeIGEL, A. u. WOLFF, B. (2006): Bodenzustandserhebung im Wald (BZE II), Arbeitsanleitung für die Außenaufnahmen. Hrsg. BMELV. Berlin, $413 \mathrm{~S}$.

WEST, P. W. (2009): Tree and forest measurement - Second Edition. Springer Verlag, Berlin, Heidelberg, 190 S.

Wirth, C.; Schulze, E.-D.; Schwalbe, G.; TOMczyK, S.; Weber, G.-E. u. WeLLER, E. (2004a): Dynamik der Kohlenstoffvorräte in den Wäldern Thüringens. Mitteilungen / Thüringer Landesanstalt für Wald, Jagd und Fischerei, 23, $308 \mathrm{~S}$.

WirTh, C.; SCHUMACHER, J. u. SCHUlZE, E.-D. (2004b): Generic biomass functions for Norway spruce in Central Europe - a meta-analysis approach toward prediction and uncertainty estimation. Tree Physiology, 24, 121

WUTZLER, T.; WiRTH, C. u. SCHUMACHER, J. (2008): Generic biomass functions for Common beech (Fagus sylvatica) in Central Europe: predictions and components of uncertainty. Can. J. For. Res., 38, 1661-1675

ZeLL, J. (2008): Methoden für die Ermittlung, Modellierung und Prognose der Kohlenstoffspeicherung in Wäldern auf Grundlage permanenter Großrauminventuren. Dissertation, Fakultät für Forst- und Umweltwissenschaften der Albert-Ludwigs-Universität zu Freiburg im Breisgau, 162 S. 
Zell, J.; KÄNDler, G. u. HANEWInKel, M. (2009): Predicting constant decay rates of coarse woody debris - A meta-analysis approach with a mixed model. Ecological Modelling, 220, 904-912

ZiAnis, D.; MuUkKONEN, P.; MÄKIPÄÄ, R. u. MENCUCCINI, M. (2005): Biomass and stem volume equations for tree species in Europe. Silva Fennica Monographs, 4, 63

ZMP (2008): ZMP-Marktbilanz Forst und Holz 2008. ZMP Zentrale Markt- und Preisberichtsstelle, Bonn, 145 S. 


\section{Autoren}

\section{René Wördehoff}

Nordwestdeutsche Forstliche Versuchsanstalt

Grätzelstraße 2

D-37079 Göttingen

Rene.Woerdehoff@nw-fva.de

\section{Prof. Dr. Hermann Spellmann}

Nordwestdeutsche Forstliche Versuchsanstalt

Grätzelstraße 2

D-37079 Göttingen

Hermann.Spellmann@nw-fva.de

\section{Dr. Jan Evers}

Nordwestdeutsche Forstliche Versuchsanstalt

Grätzelstraße 2

D-37079 Göttingen

Jan.Evers@nw-fva.de

\section{Prof. Dr. Jürgen Nagel}

Nordwestdeutsche Forstliche Versuchsanstalt

Grätzelstraße 2

D-37079 Göttingen

Juergen.Nagel@nw-fva.de 


\section{Anhang}

Übersicht über die verglichenen Biomassefunktionen zur Berechnung der lebenden Baumbiomasse

\begin{tabular}{|c|c|c|c|c|}
\hline Autor & BA & Komp. & Region & Funktion \\
\hline BARTELINK 1997 & $\mathrm{Bu}$ & OB & NL & $0.0306^{*}\left(\mathrm{BHD}^{\wedge} 2.347\right)^{*}\left(\mathrm{H}^{\wedge} 0.59\right)$ \\
\hline BOLTE et al. 2004 & $\mathrm{Bu}$ & Wurzel & $\mathrm{D}, \mathrm{NI}$ & $\exp (-4.00+2.32 * \ln (\mathrm{BHD}))$ \\
\hline BOLTE et al. 2004 & $\mathrm{Fi}$ & Wurzel & $\mathrm{D}, \mathrm{NI}$ & $\exp (-5.59+2.79 * \ln (\mathrm{BHD}))$ \\
\hline CiENCIALA et al. 2006 & $\mathrm{Ki}$ & OB & $\mathrm{CZ}$ & $0.03191 *\left(\mathrm{BHD}^{\wedge} 1.89823\right) *\left(\mathrm{H}^{\wedge} 0.89868\right)$ \\
\hline CiEnCiala et al. 2008 & $\mathrm{Ei}$ & OB & $\mathrm{CZ}$ & $\exp (-3.069+2.137 * \ln (\mathrm{BHD})+0.661 * \ln (\mathrm{H}))$ \\
\hline CiEnCIALA et al. 2005 & $\mathrm{Bu}$ & OB & $\mathrm{CZ}$ & $0.047 *\left(\mathrm{BHD}^{\wedge} 2.121\right)^{*}\left(\mathrm{H}^{\wedge} 0.697\right)$ \\
\hline FIEDLER 1986 & $\mathrm{Fi}$ & OB & $\mathrm{D}$ & $-43.13+2.25 * \mathrm{BHD}+0.452 * \mathrm{BHD}^{\wedge} 2$ \\
\hline HOCHBICHLER 2002 & $\mathrm{Bu}$ & OB & A & $\exp (-2.872+2.095 * \ln (\mathrm{BHD})+0.678 * \ln (\mathrm{H})$ \\
\hline HOCHBICHLER 2002 & $\mathrm{Ei}$ & OB & A & $\exp (-0.883+2.140 * \ln (\mathrm{BHD}))$ \\
\hline $\begin{array}{l}\text { LE GOFF u. OTTORINI } \\
2001\end{array}$ & $\mathrm{Bu}$ & Wurzel & $\mathrm{F}$ & $\exp (-3.8219+2.5382 * \ln (\mathrm{BHD}))$ \\
\hline MUKKONEN 2007 & $\mathrm{Ki}$ & OB & $\begin{array}{l}\text { temp. und boreale } \\
\text { Z. Europas }\end{array}$ & $\exp \left(-2.668+10.745^{*}(\mathrm{BHD} /(\mathrm{BHD}+8.062))\right)$ \\
\hline MUUKKONEN 2007 & $\mathrm{Bu}$ & OB & $\begin{array}{l}\text { temp. und boreale } \\
\text { Z. Europas }\end{array}$ & $\exp (0.006+10.933 *(\mathrm{BHD} /(\mathrm{BHD}+21.216)))$ \\
\hline MUUKKONEN 2007 & $\mathrm{Fi}$ & OB & $\begin{array}{l}\text { temp. und boreale } \\
\text { Z. Europas }\end{array}$ & $\exp \left(-1.694+10.825^{*}(\mathrm{BHD} /(\mathrm{BHD}+11.816))\right)$ \\
\hline MUUKKONEN 2007 & $\mathrm{Ei}$ & OB & $\begin{array}{l}\text { temp. und boreale } \\
\text { Z. Europas }\end{array}$ & $\exp (-0.604+10.677 *(\mathrm{BHD} /(\mathrm{BHD}+15.900)))$ \\
\hline PoEPPEL 1989 & $\mathrm{Fi}$ & OB & DDR & $-60.55702+5.46558 * \mathrm{BHD}+0.27567 * \mathrm{BHD}^{\wedge} 2$ \\
\hline POEPPEL 1989 & $\mathrm{Fi}$ & OB & DDR & $-283.17413+26.32334 * \mathrm{BHD}+(-0.12856) * \mathrm{BHD}^{\wedge} 2$ \\
\hline POEPPEL 1989 & $\mathrm{Fi}$ & OB & DDR & $-142.60881+13.63896 * \mathrm{BHD}+0.12593 * \mathrm{BHD}^{\wedge} 2$ \\
\hline PRETZSCH 2000 & $\mathrm{Bu}$ & OB & $\mathrm{D}, \mathrm{NI}$ & $0.1143^{*}\left(\mathrm{BHD}^{\wedge} 2.5030\right)$ \\
\hline PRETZSCH 2000 & $\mathrm{Fi}$ & OB & $\mathrm{D}$ & $0.0442 * \mathrm{BHD}^{\wedge} 2.6597$ \\
\hline WIRTH et al. 2004b & $\mathrm{Fi}$ & Nadeln & Zentraleuropa & $\exp (-3.19632+1.91620 * \ln (\mathrm{BHD}))$ \\
\hline WIRTH et al. 2004b & $\mathrm{Fi}$ & Äste & Zentraleuropa & $\exp (-3.96201+2.25520 * \ln (\mathrm{BHD}))$ \\
\hline
\end{tabular}

Beiträge aus der NW-FVA, Band 6, 2011 


\begin{tabular}{|c|c|c|c|c|}
\hline WIRTH et al. 2004b & $\mathrm{Fi}$ & $\begin{array}{l}\text { trockene } \\
\text { Äste }\end{array}$ & Zentraleuropa & $\exp (-3.22406+1.67320 * \ln (\mathrm{BHD}))$ \\
\hline WIRTH et al. 2004b & $\mathrm{Fi}$ & Stamm & Zentraleuropa & $\exp (-2.50602+2.44277 * \ln (\mathrm{BHD}))$ \\
\hline WIRTH et al. 2004b & $\mathrm{Fi}$ & Wurzel & Zentraleuropa & $\exp (-5.37891+4.08262 * \ln (\mathrm{BHD}))$ \\
\hline WUTZLER et al. 2008 & $\mathrm{Bu}$ & OB & $\mathrm{D}$ & $0.0523 *\left(\mathrm{BHD}^{\wedge} 2.12\right)^{*}\left(\mathrm{H}^{\wedge} 0.655\right)$ \\
\hline WUTZLER et al. 2008 & $\mathrm{Bu}$ & Wurzel & $\mathrm{D}$ & $0.0282 *\left(\mathrm{BHD}^{\wedge} 2.39\right)$ \\
\hline ZELL 2008 & $\mathrm{Bu}$ & $\mathrm{OB}$ & $\mathrm{D}$ & $0.0365^{*}\left(\mathrm{BHD}^{\wedge} 2.1082\right) *\left(\mathrm{H}^{\wedge} 0.7696\right)$ \\
\hline ZELL 2008 & $\mathrm{Fi}$ & OB & $\mathrm{D}$ & $0.0673^{*}\left(\mathrm{BHD}^{\wedge} 1.9378\right)^{*}\left(\mathrm{H}^{\wedge} 0.6382\right)$ \\
\hline ZELL 2008 & $\mathrm{Ki}$ & OB & $\mathrm{D}$ & $0.058^{*}\left(\mathrm{BHD}^{\wedge} 2.034\right) *\left(\mathrm{H}^{\wedge} 0.637\right)$ \\
\hline ZELL 2008 & $\mathrm{Ei}$ & OB & $\mathrm{D}$ & $0.121 *\left(\mathrm{BHD}^{\wedge} 2.435\right)$ \\
\hline ZIANIS et al. 2005 & $\mathrm{Ki}$ & $\mathrm{OB}$ & $\mathrm{CZ}$ & $0.1182 *\left(\mathrm{BHD}^{\wedge} 2.3281\right)$ \\
\hline ZIANIS et al. 2005 & $\mathrm{Ki}$ & OB & $\mathrm{N}$ & $\left(200.87186 *\left(\mathrm{BHD}^{\wedge} 2\right)+124.6808 *\left(\left(\mathrm{BHD}^{\wedge} 2\right)-49\right)\right) / 1000$ \\
\hline ZIANIS et al. 2005 & $\mathrm{Ki}$ & OB & PL & $\exp (-2.001+1.943 * \log (\mathrm{BHD}))$ \\
\hline ZIANIS et al. 2005 & $\mathrm{Ki}$ & $\mathrm{OB}$ & UK & $\left(10^{\wedge}(0.981+2.289 * \log 10(\mathrm{pi} * \mathrm{BHD}))\right) / 1000$ \\
\hline
\end{tabular}

$\begin{array}{ll}\text { A } & \text { Österreich } \\ \text { CZ } & \text { Tschechische Republik } \\ \text { D } & \text { Deutschland } \\ \text { DDR } & \text { Deutsche Demokratische Republik } \\ \text { F } & \text { Frankreich } \\ \text { FIN } & \text { Finnland } \\ \text { FW } & \text { Feinwurzel } \\ \text { NI } & \text { Niedersachsen } \\ \text { NL } & \text { Niederlande } \\ \text { OB } & \text { oberirdische Biomasse } \\ \text { S } & \text { Schweden } \\ \text { UK } & \text { Vereinigtes Königreich von Großbritannien und Nordirland }\end{array}$




\section{Beiträge aus der Nordwestdeutschen Forstlichen Versuchsanstalt}

Band

1 (2007) Clusterstudie Forst und Holz Niedersachsen. Burkhard Rüther, Jan Hansen, Agatha Ludwig, Hermann Spellmann, Jürgen Nagel, Bernhard Möhring, Matthias Dieter. 92 S.

2 (2008) Die Waldkiefer - Fachtagung zum Baum des Jahres 2007. Nordwestdeutsche Forstliche Versuchsanstalt (Hrsg.). 98 S.

3 (2008) Ergebnisse angewandter Forschung zur Buche. Nordwestdeutsche Forstliche Versuchsanstalt (Hrsg.). 343 S.

4 (2008) Ergebnisse des westdeutschen IUFRO-Küstentannen-Provenienzversuches im Alter 27. Hans-Martin Rau, Armin König, Wolfhard Ruetz, Hendrik Rumpf, Egbert Schönfelder. $62 \mathrm{~S}$.

5 (2010) Fichtenherkunftsversuch von 1962 und IUFRO-Fichtenherkunftsversuch von 1972. Ergebnisse von mehr als 30jähriger Beobachtung in Deutschland. Mirko Liesebach, Hans-Martin Rau, Armin O. König. 467 S.

6 (2011) Kohlenstoffstudie Forst und Holz Niedersachsen. René Wördehoff, Hermann Spellmann, Jan Evers, Jürgen Nagel. 92 S.

Alle Bände der „Beiträge aus der NW-FVA“ sind auch als freie Onlineversion über die Homepage der NW-FVA (www.nw-fva.de), des Verlages sowie über den OPAC der Niedersächsischen Staats- und Universitätsbibliothek

(http://www.sub.uni-goettingen.de) erreichbar und dürfen gelesen, heruntergeladen sowie als Privatkopie ausgedruckt werden. Es gelten die Lizenzbestimmungen der Onlineversion. Es ist nicht gestattet, Kopien oder gedruckte Fassungen der freien Onlineversion zu veräußern. 
Die Speicherung von Kohlenstoff in Wäldern ist klimapolitisch ein hochaktuelles Thema, das die niedersächsische Landesregierung mit der vorliegenden Studie aufgreift. Auf der Basis von Inventurdaten und Szenarienrechnungen werden die Mengen an Kohlenstoff hergeleitet, die heute bzw. künftig in niedersächsischen Wäldern und in den aus niedersächsischem Holz hergestellten Produkten gespeichert werden. Die Kohlenstoffstudie richtet sich an alle Betriebe der Forst- und Holzwirtschaft, politische Entscheidungsträger und die interessierte Öffentlichkeit. Sie liefert wichtige Argumente im Kyoto-Folgeprozess und zeigt die Auswirkungen verschiedener waldbaulicher Strategien auf die Kohlenstoffspeicherung in Wäldern und Forstprodukten auf. 